\title{
OPERADORES QUE ALCANZAN SU NORMA
}

MEMORIA DE LICENCIATURA

\author{
MANUEL RUIZ GALÁN
}

UNIVERSIDAD DE GRANADA, 1994 
FACULTAD DE CIENCIAS

DEPARTAMENTO DE ANÁLISIS MATEMÁTICO

\section{OPERADORES \\ QUE ALCANZAN SU NORMA}

MANUEL RUIZ GALÁN

UNIVERSIDAD DE GRANADA, 1994 
Memoria de Licenciatura dirigida por la Doctora $D^{a}$ Maria Dolores Acosta Vigil, profesora del Departamento de Análisis Matemático, defendida por D. Manuel Ruiz Galán el día 7 de marzo de 1994, ante el tribunal formado por los siguientes profesores: D. Manuel Barros Díaz (Presidente), Da María Dolores Acosta Vigil (Secretaria) y D. Rafael Payá Albert (Vocal). Obtuvo la calificación de Sobresaliente por Unanimidad. 
A mis padres y mi hermano 


\section{Indice}

Introducción. $\quad$ ix

1 El Teorema de Bishop-Phelps. 1

2 El Teorema de Lindenstrauss-Zizler. 21

3 Las propiedades "A" y "B" de Lindenstrauss. 31

3.A. El Teorema de renormación de Partington. .......... 32

3.B. El Teorema de renormación de Srhachermayer. ........ 43

4 Operadores que alcanzan su norma y la propiedad de Radon-Nikodym. $\quad 69$

4.A. Principio de optimización no lineal de Bourgain-Stegall. .. 70

4.B. El Teorema de Bourgain-Huff. .................. 84

5 Algunos resultados en espacios clásicos. $\quad 99$

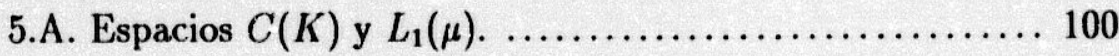

5.B. Espacios de sucesiones $l_{p}(1<p<\infty)$. ........... 136

5.C. El problema de Bishop-Phelps para la clase de los operadores compactos. ............................ 145

$\begin{array}{ll}\text { Bibliografía. } & 163\end{array}$ 


\section{Introducción.}

En la elaboración de la presente memoria hemos pretendido recoger de una manera sistemática las respuestas que se conocen en la actualidad al problema de la densidad de los operadores que alcanzan su norma, evitando, en la medida de lo posible, usar resultados sin incluir su demostración, intentando así conseguir una cierta inúependencia en la exposición. Hernos tratado también de presentar muchos de los problemas abiertos relacionados con el tema, procurando que éstos aparezcan en el contexto que les corresponde.

El origen del problema de los operadores que alcanzan la norma se remonta a un articulo de E. Bishop y R.R. Phelps ([4]) publicado en 1.961 , en el que estos autores prueban que, en un espacio de Banach cualquiera, el conjunto de los funcionales (lineales y continuos) que alcanzan su norma es denso en el dual, para la tonología de la norma. Dos años más tarde, aparece un nuevo trabajo de estos mismos autores, en el que se generaliza este resultado en términos de funcionales y puntos de soporte de un subconjunto convexo y cerrado de un espacio de Banach (en particular, tomando como subconjunto la bola unidad del espacio, obtenemos funcionales que alcanzan la norma). En el primer capítulo de esta memoria ofrecemos una demostración de este resultado (Teorema de Bishop-Phelps) distinta de la original que dieron sus autores; nosotros hemos seguido la que aparece en el texto de R.R. Phelps [31], en la que el mencionado teorema se obtiene a partir de un principio de optimización perturbada, el Principio Variacional de Ekeland, consiguiendo de hecho una mejora cuantitativa del resultado obtenida por B. Bollobás en 1.970 ([6]).

Nótese que el Teorema de Bishop-Phelps afirma que el conjunto de los 
operadores (lineales y continuos) de un espacio de Banach $X$ en el cuerpo base $\mathbb{K}$ que alcanzan la norma, es denso en el espacio de los operadores de $X$ en $\mathbb{K}$. En el primer artículo donde aparece este resultado, E. Bishop y R.R. Phelps plantean si el mismo hecho será cierto en general, cambiando el papel de $\mathbf{K}$ por el de un espacio de Banach. Más concretamente, plantean la siguiente cuestión, que hemos recogido en el Capítulo II:

¿Para qué espacios de Banach $X$ e $Y$ el conjunto $N A(X, Y)$ de los operadores que alcanzan su norma es denso en el espacio $L(X, Y)$ de todos los operadores de $X$ en $Y$ para la topología de la norma?

Las primeras respuestas al problema aparecen en un relevante trabajo de J. Lindenstrauss ([27]), publicado en 1.963. Er primer lugar, este autor prueba que, en general, la respuesta no puede ser afirmativa. De hecho, tomando como $X=c_{0}$, el espacio de las sucesiones convergentes a cero, dotado de su norma usual, y como espacio $Y$ una renormación estrictamente convexa de $c_{0}$, los elementos de $N A(X, Y)$ son, en este caso, operadores de rango finito, $y$, por tanto, para estos espacios $\overline{N A(X, Y)} \neq L(X, Y)$. Además prueba que el conjunto de los operadores cuyo segundo adjunto alcanza su norma es áenso siempre en el espacio de los operadores. V. Zizler mejora diez años más tarde este resultado, al poner de manifiesto que de hecho ya hay densidad para los operadores cuyo adjunto alcanza su norma (Teorema 2.6) con lo que, en particular, todo espacio reflexivo satisface la propiedad $A$, lo que ya se deducía del Teorema de Lindenstrauss. Observemos que, mientras que el problema planteado es, aparentemente, de naturaleza geométrica, hasta ahora se ha conseguido respuesta afirmativa imponiendo una hipótesis de carácter isomórfico, la reflexividad.

Teniendo en cuenta que la pregunta inicial de E. Bishop y R.R. Phelps es demasiado general como para tener respuesta afirmativa, J. Lindenstrauss introduce las propiedades $A$ y $B$ : un espacio de Banach $X$ verifica 
la propiedad $A$ (resp. $B$ ) si para todo espacio de Banach $Y, N A(X, Y)$ (resp. $N A(Y, X)$ ) es denso en norma en $L(X, Y)$ (resp. $L(Y, X)$ ). Este mismo autor da condiciones suficientes para que un espacio tenga $A$ o $B$. Hasta el momento no se conocen caracterizaciones satisfactorias de las propiedades $A$ y $B$, únicamente algunas condiciones suficientes. En cambio, sí está más estudiada la cuestión de si un espacio de Banach se puede renormar equivalentemente para conseguir alguna de las dos propiedades de J. Lindenstrauss. De estos resultados nos ocuparemos en el Capítulo III. En un artículo publicado en 1.982, J. Partington establece que todo espacio de Banach es isomorfo a otro verificando la propiedad $B([29])$, resultado que aparece recogido en la memoria en el Corolario 3.11. En cambio, para la propiedad $A$ no se dispone de un resultado a plena generalidad. W. Schachermayer probó que todo espacio de Banach débilmente compactamente generado es isomorfo a otro con la propiedad $A$ ([34]). Con idéntica demostración también se obtiene que todo espacio que admita una resolución proyectiva de la identidad es isomorfo a otro que tiene la propiedad $A$ (Teorema 3.34 ) y como caso particular, aparece el resultado de W. Schachermayer para espacios débilmente compactamente generados.

El Capítulo IV relaciona el problema de los operadores que alcanzan la norma con una propiedad bastante estudiada en la teoría de espacios de Banach, la propiedad de Radon-Nikodym. Haciendo uso de un principio de optimización perturbada, el Principio de optimización no lineal de Bourgain-Stegall, se prueba que dicha propiedad es suficiente para la propiedad $A$ (Corolario 4.12), hecho que había sido observado por $\mathrm{J}$. Bourgain ([8]), y como consecuencia de un resultado de Bourgain-Huff, que si $X$ es un espacio de Banach, tal que cualquier espacio isomorfo a $X$ tiene la propiedad $A$ (igual $\operatorname{con} B$ ), entonces $X$ verifica la propiedad de Radon-Nikodym (Corolarios 4.21 y 4.20).

Además de los resultados anteriores, han ido apareciendo varios tra- 
bajos que dan respuesta al problema planteado para ciertos espacios concretos, todos ellos espacios clásicos. Los que han sido considerados por nosotros más relevantes aparecen recogidos en el Capítulo V.

J. Johnson y J. Wolfe prueban en 1.979 que el conjunto $N A(C(K), C(S))$ es denso en $L(C(K), C(S))$, donde $K$ y $S$ son espacios topológicos compactos y de Hausdorff. Nos obstante, los espacios del tipo $C(K)$ no siempre tienen la propiedad $B$ : los mismos autores construyen un espacio métrico compacto $S$ tal que los operadores de $L_{1}[0,1]$ en $C(S)$ que alcanzan la norma no forman un subconjunto denso del correspondiente espacio de operadores (por tanto, $L_{1}[0,1]$ no verifica la propiedad $A$ ). Finalmente, un resultado de W. Schachermayer (1.983) afirma que $N A\left(C[0,1], L_{1}[0,1]\right)$ es denso en $L\left(C[0,1], L_{1}[0,1]\right)$ y otro de W. Gowers $(1.990)$ que los espacios de sucesiones $l_{p}(1<p<\infty)$ no satisfacen la propiedad $B$, resolviendo así un problema propuesto por $J$. Johnson y J. Wolfe en [24].

A la serie de resultados conocidos relativos a espacios concretos, hay que añadir algunas respuestas al problema considerando ciertas clases de espacios de operadores. En este sentido, J. Johnson y J. Wolfe ([24]) logran probar que el conjunto de los operadores compactos de $X$ en $Y$ que alcanzan la norma es denso en el espacio de los operadores compactos, siempre y cuando, alguno de los dos espacios $(X \circ Y)$ sea del tipo $C(K)$ o $L_{1}(\mu)$ (Teorema 5.28 y Teorema 5.29), y sugieren el siguiente problema:

¿Existe algún par de espacios de Banach $X$ e $Y$ para el cual los operadores compactos de $X$ en $Y$ que alcanzan su norma no forman un subconjunto denso del espacio de los operadores compactos?

No tenemos respuesta, ni siquiera en el caso en que $Y$ sea finito dimensional, es decir, no sabemos si hay algún espacio de dimensión finita que no tenga la propiedad $B$. La dificultad se ve acentuada aún más cuando comprobamos que únicamente contamos con una reducida gama 
de ejemplos de espacios finito dimensionales verifcando la propiedad $B$. Entre ellos se encuentran aquéllos que son reales y cuya bola unidad es un poliedro (Teorema 3.4). Así, y a modo de ejemplo llamativo,

\section{¿Verifica $\mathbf{R}^{2}$ con la norma euclídea la propiedad $B$ ?}

Otra cuestión que hoy por hoy se encuentra si resolver es la siguiente:

\section{$\dot{\delta}$ Tiene $l_{1}$ la propiedad $B$ ?}

Los problemas anteriores no son más que una pequeña muestra de los muchos que en la actualidad permanecen abiertos. Este hecho, junto con la profundidad de muchos de los resultados comentados anteriormente, pone de manifiesto la vitalidad y complejidad que el problema de los operadores que alcanzan su norma encierra.

Por último, quisiera expresar, en primer lugar, mi más sincero agradecimiento a la Directora de esta memoria, María Dolores Acosta Vigil, sin cuya dedicación y entusiasmo permanentes no habría sido posible la elaboración de la misma.

También deseo manifestar mi gratitud hacia los profesores Rafael Payá Albert y Antonio Martínez Cegarra por sus acertadas indicaciones, a todos los miembros del Departamento de Análisis Matemático de la Universidad de Granada, en especial a su Director, Pedro Jiménez Garijo, y a Juan Carlos Cabello Piñar, por su acogida y las enormes facilidades que me han brindado, a Victoriano Ramírez González, Director del Departamento de Matemática Aplicada de la Universidad de Granada, por el apoyo que me ha prestado, asi como a todos y cada uno de los que de una forma u otra han contribuido a la realización de esta memoria. 


\section{Capítulo 1}

\section{El Teorema de Bishop-Phelps.}

Este primer capítulo se dedica por entero al estudio del Teorema de Bishop-Phelps, resultado que dió origen al problema de la densidad de los operadores que alcanzan la norma. La demostración que damos usa como principal ingrediente el Principio Variacional de Ekeland. Más tarde, pondremos de manifiesto que una versión del Teorema de BishopPhelps para espacios de Banach complejos que involucra la estructura complejo-lineal del espacio permanece hoy en día planteada como una cuestión abierta.

En esta memoria seguiremos la notación usual: si $X$ es un espacio normado, representaremos por $X^{*}$ al dual topológico de $X$, es decir, el espacio de Banach de los funcionales (lineales y continuos) en $X . S_{X}$ y $B_{X}$ serán respectivamente la esfera unidad y la bola cerrada unidad de $X$. Diremos que un funcional $x^{*}$ alcanza la norma si el supremo que define su norma es un máximo, esto es, existe un vector $x_{0}$ en $X$ de norma uno tal que $x^{*}\left(x_{0}\right)=\left\|x^{*}\right\|$. 
Es claro que si $X$ es un espacio de Banach reflexivo, todo funcional alcanza la norma. B.C. James ([22]) prueba en 1.957 que, en caso separable, esta propiedad caracteriza la reflexividad. Es imposible, por tanto, pretender que en un espacio de Banach arbitrario, cualquier funcional alcance la norma, si bien el Teorema de Hahn-Banach garantiza la existencia de este tipo de funcionales. E. Bishop y R.R. Phelps se plantearon el problema de si, en general, tales funcionales abundan en un espacio de Banach. En un primer trabajo publicado en 1.961 ([4]) estos autores prueban que para cualquier espacio de Banach $X$, el conjunto de los funcionales que alcanzan la norma es denso en $X^{*}$. Como muestra [30], la complitud del espacio es esencial en este resultado.

En el Teorema de Bishop-Phelps juegan un papel fundamental los conceptos de funcional de soporte y punte de soporte, que motivamos a continuación. Detengámonos por un momento en la interpretación geométrica del hecho de que un funcional alcance su norma: si $x^{*}$ es un tal funcional, hay un elemento $x_{0}$ en $B_{X}$ verificando

$$
\operatorname{Re} x^{*}\left(x_{0}\right) \geq \operatorname{Re} x^{*}(x), \forall x \in B_{X}
$$

es decir, la función $\operatorname{Re} x^{*}$ definida en $B_{X}$ alcanza su máximo. Por tanto, el hiperplano

$$
\left\{x \in X: \operatorname{Re} x^{*}(x)=\operatorname{Re} x^{*}\left(x_{0}\right)\right\}
$$

contiene a $x_{0}$ y deja a $B_{X}$ incluida en uno de los dos semiespacios cerrados que determina.

1.1 Definición. Sea $C$ un subconjunto no vacio, convexo y cerrado de un espacio de Banach $X$. Un vector $x_{0} \in C$ es un punto de soporte de $C$ si existe un funcional no nulo $x^{*} \in X^{*}$ verificando

$$
\operatorname{Re} x^{*}\left(x_{0}\right) \geq \operatorname{Re} x^{*}(x), \forall x \in C,
$$


es decir, el funcional Re $x^{*}$ alcanza en $x_{0}$ el supremo de sus valores en $C$. Si esto ocurre, se dice que $x^{*}$ es un funcional de soporte de $C$ (en $\left.x_{0}\right)_{0}$ también que $x^{*}$ soporta a $C$ en $x_{0}$.

Como consecuencia del Teorema de separación de conjuntos convexos, si un subconjunto no vacío, convexo y cerrado $C$ de un espacio de Banach tiene interior no vacío, entonces todo punto de su frontera es un punto de soporte de $C$. Si no exigimos a $C$ la condición de interior no vacío, ¿se puede afirmar algo sobre la abundancia de tales puntos? iY la de funcionales? El Teorema de Bishop-Phelps da respuesta precisa a estas dos cuestiones.

La demostración que presentamos aquí no es la original de E. Bishop y R.R. Phelps; nosotros hemos seguido el texto de R.R. Phelps [32], donde el resultado se obtiene a partir del Teorema de Bronsted-Rockafellar, que a su vez es consecuencia del Principio Variacional de Ekeland. Por un lado, nos parece interesante ofrecer una demostración distinta de la original y por otro, tendremos la oportunidad de familiarizarnos con algunos resultados sobre optimización perturbada, de interés en sí mismos, y dentro de los cuales podemos enmarcar el problema de los operadores que alcanzan la norma.

Para probar el primero de los resultados (Teorema de Ekeland) haremos uso de las siguientes nociones:

1.2 Definición. Sea $f$ una función definida en un espacio topológico de Hausdorff $\Omega$ que toma valores en $\mathbb{R} \cup\{\infty\}$.

i) La epigráfica de $\mathrm{f}$ es el subconjunto de $\Omega \times \mathbb{R}$ definido por

$$
\operatorname{epi}(f)=\{(\omega, r) \in \Omega \times \mathbb{R}: r \geq f(x)\} .
$$


ii) La función $\mathrm{f}$ es inferiormente semicontinua si $\left.\left.f^{-1}(]-\infty, \alpha\right]\right)$ es cerrado, para cualquier número real $\alpha, o$, equivalentemente, si epi $(f)$ es cerrado en $\Omega \times \mathbb{R}$.

iii) El dominio efectivo de $f$ es el subconjunto de $\Omega$ dado por

$$
\operatorname{dom}(f)=\{\omega \in \Omega: f(\omega)<\infty\}
$$

y f es propia cuando $\operatorname{dom}(f) \neq \emptyset$.

Nótese que la suma de dos funciones inferiormente semicontinuas es obviamente inferiormente semicontinua.

1.3 Ejemplo. Sea $C$ un subconjunto de $\Omega$ y definamos la función indicadora de $C \delta_{C}$ como

$$
\delta_{C}(\omega)= \begin{cases}0, & \text { si } \omega \in C \\ \infty, & \text { en otro caso }\end{cases}
$$

Obsérvese que $\delta_{C}$ es inferiormente semicontinua si, y sólo si, el conjunto $C$ es cerrado.

Nuestro primer objetivo es demostrar el Teorema de Ekeland. Con este fin probaremos un lema previo que abstrae la misma idea geométrica de la prueba original que aparece en el Teorema de Bishop-Phelps [5, Lemma 1], aunque lo hemos enunciado en un ambiente más general que el de los espacios normados.

Sea $E$ un espacio métrico con distancia $d$ y consideremos un número real y positivo $\lambda$ y un elemento $(x, r)$ de $E \times \mathbb{R}$. Entonces representaremos por $K_{\lambda}(x, r)$ el conjunto

$$
K_{\lambda}(x, r)=\{(y, s) \in E \times \mathbb{R}: \lambda d(x, y) \leq r-s\} .
$$


Claramente $K_{\lambda}(x, r)$ es un subconjunto cerrado de $E \times \mathbb{R}$. Si la distancia $d$ procede de una norma, $K_{\lambda}(x, r)$ es un cono con origen en $(x, r)$.

1.4 Lema ([32, Lemma 3.12]). Sea $(E, d)$ un espacio métrico, $P_{2}: E \times \mathbb{R} \longrightarrow \mathbb{R}$ la proyección canónica y $A$ un subconjunto no vació y completo de $E \times \mathbb{R}$ tal que $P_{2}(A)$ está minorado. Entonces, para cada $\lambda>0$ y cada $\left(x_{0}, r_{0}\right) \in A$, existe $(z, r)$ en $A$ verificando las dos siguientes condiciones:

(i) $(z, r) \in A \cap K_{\lambda}\left(x_{0}, r_{0}\right)$

(ii) $\{(z, r)\}=A \cap K_{\lambda}(z, r)$.

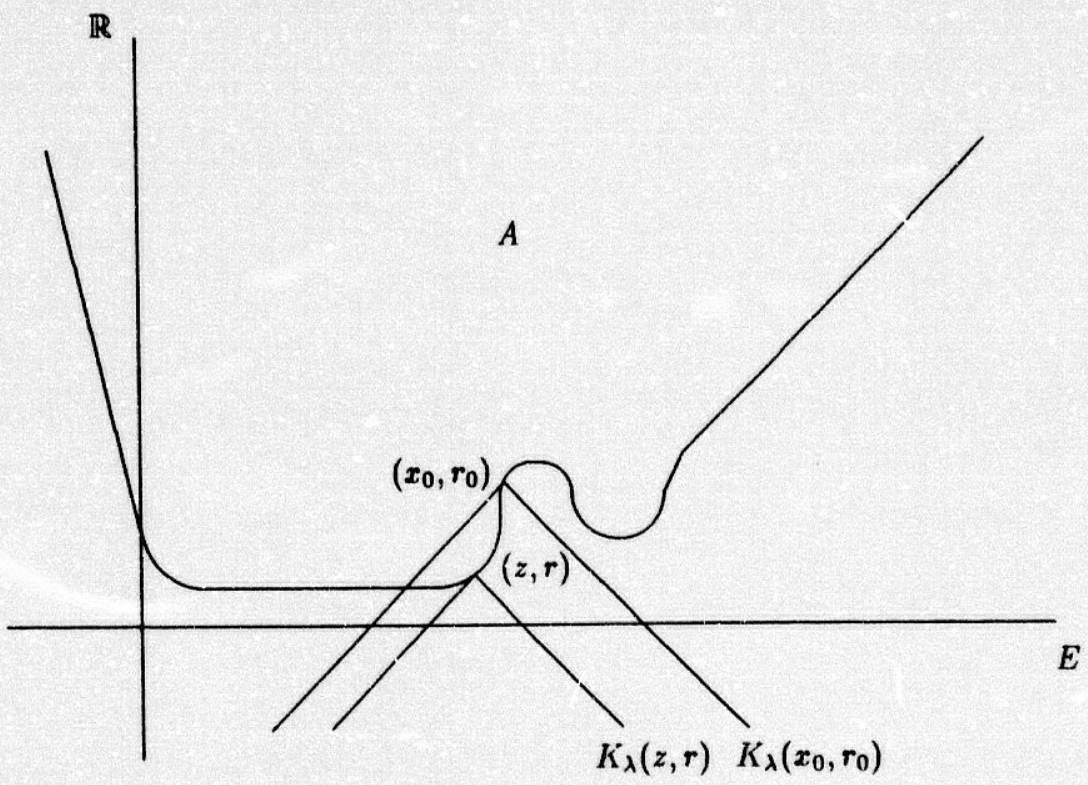




\section{Demostración. Definimos}

$$
A_{0}=A \cap K_{\lambda}\left(x_{0}, r_{0}\right)
$$

y elegimos un elemento $\left(x_{1}, r_{1}\right)$ de $A_{0}$ de modo que

$$
r_{1}<\inf P_{2}\left(A_{0}\right)+1 \text {. }
$$

Por recurrencia construimos una sucesión $\left\{A_{n}\right\}_{n \geq 0}$ de elementos de $A$ tal que para cada $n \in \mathbb{N} \cup\{0\}$

$$
\left(x_{n+1}, r_{n+1}\right) \in A_{n}:=A \cap K_{\lambda}\left(x_{n}, r_{n}\right)
$$

y

$$
r_{n+1}<\inf P_{2}\left(A_{n}\right)+\frac{1}{n+1}
$$

Nótese que, por construcción de los conjuntos $A_{n}$, para cada $n \geq 0$, $A_{n+1} \subseteq A_{n}$. Además, $\operatorname{diam}\left(A_{n+1}\right) \leq 2 / \lambda(n+1)$, ya que si $(y, s) \in A_{n+1}$ entonces, por un lado, $s \geq \inf \left(A_{n+1}\right)$ y

$$
\begin{gathered}
\lambda d\left(y, x_{n+1}\right) \leq r_{n+1}-s \leq \inf P_{2}\left(A_{n}\right)+\frac{1}{n+1}-s \leq \\
\leq \inf P_{2}\left(A_{n+1}\right)+\frac{1}{n+1}-s \leq \frac{1}{n+1}
\end{gathered}
$$

y por otro,

$$
\left|r_{n+1}-s\right|=r_{n+1}-s<\inf P_{2}\left(A_{n}\right)+\frac{1}{n+1}-s \leq \frac{1}{n+1} .
$$

El Teorema de Cantor asegura entonces la existencia de un elemento $(z, r)$ en $A$ tal que

$$
\bigcap_{n \geq 0} A_{n}=\{(z, r)\}
$$

Como $(z, r) \in A_{0}$ se tiene (i). Para comprobar (ii) observemos que si $(y, s)$ está en $A \cap K_{\lambda}(z, r)$, entonces, usando la desigualdad triangular, $(y, s) \in A_{n}, \forall n \in \mathbb{N} \cup\{0\}$ y por tanto

$$
(y, s)=(z, r)
$$


1.5 Lema (Principio Variacional de Ekeland [32, Lemma 3.13]). Sea $(E, d)$ un espacio métrico completo y $f: E \rightarrow \mathbb{R} \cup\{\infty\}$ una función propia, inferiormente semicontinua y minorada. Consideremos un número real $\varepsilon>0$ y un elemento $x_{0}$ de $E$ tales que

$$
f\left(x_{0}\right) \leq \inf f(E)+\varepsilon .
$$

Entonces, para cada $\lambda>0$ existe un elemento $z$ en $\operatorname{dom}(f)$ verificando:

(i) $\lambda d\left(z, x_{0}\right) \leq f\left(x_{0}\right)-f(z)$,

(ii) $d\left(z, x_{0}\right) \leq \varepsilon / \lambda \quad y$

(iii) $\lambda d(z, x)+f(x)>f(z)$, si $x \in E \backslash\{z\}$.

0 sea, si tomamos un elemento $x_{0} \in E$ de forma que $f\left(x_{0}\right)$ esté próximo a inf $f(E)$, entonces podemos perturbar $f$ mediante una función lipschitziana, con constante de Lipschitz arbitrariamente pequeña, para que la nueva función alcance su mínimo en un único punto $z$ cercano a $x_{0}$. Esta es la filosofía que subyace a todo principio de optimización perturbada: una función $f$ definida en un conjunto $C$ y mayorada (respectivamente minorada), no tiene que alcanzar su supremo (respectivamente ínfimo) en $C$ necesariamente. Se trata de imponer ciertas bondades a la función y al conjunto de modo que, sumando a $f$ otra conveniente función, pequeña en algún sentido, la nueva función sí alcance su supremo (respectivamente ínfimo) en $C$.

Demostración. Podemos suponer evidentemente que inf $f(E)=0$ y que $\lambda=1$. Sea pues $x_{0}$ un elemento de $E$ tal que $f\left(x_{0}\right) \leq \varepsilon$. Por ser $f$ inferiormente semicontinua, el subconjunto $A=\operatorname{epi}(f)$ de $E \times \mathbb{R}$ es cerrado. El lema anterior nos proporciona un elemento $(z, r) \in E \times \mathbb{R}$ 
satisfaciendo las dos condiciones siguientes:

$$
(z, r) \in A \cap K_{1}\left(x_{0}, f\left(x_{0}\right)\right)
$$

y

$$
\{(z, r)\}=A \cap K_{1}(z, r) .
$$

Obviamente $z \in \operatorname{dom}(f)$ por ser $f(z) \leq r$. Las afirmaciones (i) y (ii) se siguen de la siguiente cadena de desigualdades, la primera de las cuales es consecuencia de (1):

$$
d\left(x_{0}, z\right) \leq f\left(x_{0}\right)-f(z) \leq f\left(x_{0}\right) \leq \varepsilon .
$$

Para probar (iii), comprobamos antes que $f(z)=r$. En efecto; si $f(z)<r,(2)$ nos dice que $(z, f(z)) \notin K_{1}(z, r)$ y, por tanto, $f(z)>r$, lo que contradice el hecho de que $(z, r)$ esté en $A$. Tomemos ahora $x \in E \backslash\{z\}$; si $f(x)=\infty$, (iii) se satisface evidentemente. Supongamos entonces $f(x)<\infty$. La condición (2) nos asegura que

$$
(x, f(x)) \notin K_{1}(z, f(z)),
$$

es decir,

$$
d(x, z)>f(z)-f(x)
$$

A partir del Principio Variacional de Ekeland se obtiene con bastante facilidad el Teorema de Bronsted-Rockafellar, en cuyo enunciado aparece el concepto de subdiferencial de una función convexa, noción que necesita ser precisada.

1.6 Definición. Sea $C$ un subconjunto convexo de un espacio vectorial (real o complejo) $X$. Una función $f: C \rightarrow \mathbb{R} \cup\{\infty\}$ se dice convexa si

$$
f(t x+(1-t) y) \leq t f(x)+(1-t) f(y),
$$

cualesquiera que sean $x$ e $y$ en $C$ y $t \in] 0,1[$. 
Es inmediato que la convexidad de $f$ equivale a la de epi $(f)$. No menos inmediato resulta el hecho de que la función indicadora de un subconjunto convexo de un espacio vectorial es convexa.

1.7 Definición. Sea $X$ un espacio de Banach real, $C$ un subconjunto convexo de $X, f: C \longrightarrow \mathbb{R} \cup\{\infty\}$ una función convexa propia y $\varepsilon \geq 0$. La $\varepsilon$-subdiferencial de $f$ es la función multivaluada definida como sigue:

$$
\begin{gathered}
\partial_{\varepsilon} f: C \longrightarrow \mathcal{P}\left(X^{*}\right) \\
\partial_{\varepsilon} f(x)=\left\{x^{*} \in X^{*}: x^{*}(y-x) \leq f(y)-f(x)+\varepsilon, \forall y \in C\right\}
\end{gathered}
$$

si $x \in \operatorname{dom}(f)$ y

$$
\partial_{\varepsilon} f(x)=\emptyset
$$

si $x \notin \operatorname{dom}(f)$. La 0 -subdiferencial de $f$ se llama la subdiferencial y se nota $\partial f$.

Obsérvese que

$$
\partial f(x)=\bigcap_{\varepsilon>0} \partial_{\varepsilon} f(x), \forall x \in C
$$

Puede ocurrir que, aún estando $x$ en $\operatorname{dom}(f), \partial_{\varepsilon} f(x)=\emptyset$ : tómense $C=X$ espacio de Banach real infinito-dimensional, $f$ un funcional lineal en $X$ discontinuo, $x \in X$ y $\varepsilon \geq 0$; en tal caso

$$
\partial_{\varepsilon} f(x)=\emptyset \text {. }
$$

No obstante, si $C$ es un subconjunto convexo de un espacio de Banach real $X$ y $f: C \longrightarrow \mathbb{R}$ es una función inferiormente semicontinua y convexa, entonces, para cada $\varepsilon>0$ y cada $x \in C$,

$$
\partial_{\varepsilon} f(x) \neq \emptyset:
$$


epi $(f)$ es un subconjunto convexo y cerrado de $X \times \mathbf{R}$ y $(x, f(x)-\varepsilon) \notin$ epi $(f)$, por lo que, aplicando el Teorema de separación de conjuntos convexos, es inmediato obtener un funcional en $\partial_{\varepsilon} f(x)$. Sin embargo, bajo las mismas hipótesis, $\partial f(x)$ puede ser vacío, como muestra el siguiente ejemplo: se consideran el subconjunto convexo de $l_{2}$

$$
C=\left\{x \in l_{2}:|x(n)| \leq 2^{-n}, \quad \forall n \in \mathbb{N}\right\}
$$

y la función

$$
\begin{gathered}
f: C \longrightarrow \mathbb{R} \text { dada por } \\
f(x)=\sum_{n=1}^{\infty}-\left(x(n)+\frac{1}{2^{n}}\right)^{1 / 2} \quad(x \in C),
\end{gathered}
$$

que es convexa y continua. Entonces $\partial f(0)=\emptyset$, ya que si $x^{*} \in \partial f(0), n$ es un número natural y $0<t \leq 1 / 2^{n}$,

$$
-\left\|x^{*}\right\| \leq x^{*}\left(e_{n}\right) \leq \frac{1}{t}\left(f\left(t e_{n}\right)-f(0)\right) \rightarrow-\frac{1}{2}\left(2^{n}\right)^{1 / 2}(t \rightarrow 0),
$$

lo cual es imposible (tómese límite cuando $n \rightarrow \infty$ ).

El siguiente resultado afirma que, bajo ciertas hipótesis, la subdiferencial de una función convexa es no vacía en un conjunto denso de puntos.

1.8 Teorema (Teorema de Bronsted-Rockafellar [14, Chapter 3, p. 167]). Sea $C$ un subconjunto no vacio, convexo y cerrado de un espacio de Banach real $X$ y $f: C \longrightarrow \mathbf{R}$ una función inferiormente semicontinua $y$ convexa. Dados $\varepsilon>0, x_{0} \in C, x_{0}^{*} \in \partial_{\varepsilon} f\left(x_{0}\right)$ y $\lambda>0$, existen un elemento $x_{1} \in C$ y un funcional $x_{1}^{*} \in \partial f\left(x_{1}\right)$ tales que

$$
\left\|x_{1}-x_{0}\right\| \leq \varepsilon / \lambda \text { y }\left\|x_{0}^{*}-x_{1}^{*}\right\| \leq \lambda
$$

Demostración. Definimos la función

$$
\Psi: C \longrightarrow \mathbb{R}
$$


dada por

$$
\Psi(x)=f(x)-x_{0}^{*}(x)+\sup \left(x_{0}^{*}-f\right)(C) \quad(x \in C) .
$$

$\Psi$ está bien definida $\left(x_{0}^{*} \in \partial_{e} f\left(x_{0}\right)\right)$, es convexa e inferiormente semicontinua (es suma de una función inferiormente semicontinua y otra continua). Usando nuevamente que $x_{0}^{*} \in \partial_{e} f\left(x_{0}\right)$, es decir, la desigualdad

$$
x_{0}^{*}(y)-x_{0}^{*}\left(x_{0}\right) \leq f(y)-f\left(x_{0}\right)+\varepsilon, \forall y \in C,
$$

se tiene

$$
\Psi\left(x_{0}\right) \leq \Psi(y)+\varepsilon, \forall y \in C
$$

y por tanto

$$
\Psi\left(x_{0}\right) \leq \inf \Psi(C)+\varepsilon .
$$

Sea la función $\hat{\Psi}: X \longrightarrow \mathbb{R}$ definida por

$$
\hat{\Psi}(x)= \begin{cases}\Psi(x), & \text { si } x \in C \\ \infty, & \text { en otro caso }\end{cases}
$$

Como epi $(\hat{\Psi})=\operatorname{epi}(\Psi), \hat{\Psi}$ es también una función convexa e inferiormente semicontinua; además está acotada inferiormente. Si $\lambda$ es un número real positivo arbitrario, teniendo en cuenta (1), el Principio Variacional de Ekeland (Lema 1.5) nos proporciona un elemento $x_{1} \in C$ tal que

$$
\left\|x_{0}-x_{1}\right\| \leq \varepsilon / \lambda
$$

y

$$
\lambda\left\|x-x_{1}\right\|+\hat{\Psi}(x)>\hat{\Psi}\left(x_{1}\right), \quad \forall x \in X \backslash\left\{x_{1}\right\}
$$

es decir,

$$
\hat{\Psi}\left(x_{1}+y\right)-\hat{\Psi}\left(x_{1}\right)>-\lambda\|y\|, \quad \forall y \in X \backslash\{0\}
$$

Consideramos en $X \times \mathbf{R}$ los subconjuntos $E$ y $F$ dados por:

$$
E=\left\{(y, \alpha) \in X \times \mathbb{R}: \alpha \geq \hat{\Psi}\left(x_{1}+y\right)-\hat{\Psi}\left(x_{1}\right)\right\}
$$


y

$$
F=\{(z, \beta) \in X \times \mathbb{R}: \beta<-\lambda\|z\|\} .
$$

Evidentemente ambos conjuntos son no vacíos, disjuntos, cada uno de ellos es convexo ( $E$ es la epigráfica de una función convexa) y además, $F$ es abierto. Por tanto, uasndo el Teorema de separación de Hahn-Banach, sabemos que existen un funcional $x^{*} \in X^{*}$ y un número real $\rho$ tales que

$$
x^{*}(z)+\rho \beta<x^{*}(y)+\rho \alpha, \forall(y, \alpha) \in E,(z, \beta) \in F .
$$

Si $z \in X$ es un elemento no nulo, podemos aplicar la desigualdad anterior a los elementos $( \pm z,-2 \lambda\|z\|) \in F$ y $(0,0) \in E$, obteniendo

$$
\pm x^{*}(z)-2 \rho \lambda\|z\|<0
$$

$y$, por tanto, $\rho$ es positivo. Notando $y^{*}=-\frac{1}{\rho} x^{*}$ y usando (3) para $(y, \alpha)=(0,0)$ se tiene

$$
\beta<y^{*}(z), \forall(z, \beta) \in F,
$$

desigualdad válida, por tanto, siempre que $\beta<-\lambda\|z\|$, luego $-\lambda<y^{*}(z /\|z\|), \forall z \neq 0$, de donde

$$
\left\|y^{*}\right\| \leq \lambda \text {. }
$$

Particularizando (3) para $z=0$ y tomando límite $(\beta \rightarrow 0)$, podemos concluir que

$$
y^{*}(y) \leq \alpha, \forall(y, \alpha) \in E,
$$

y eligiendo $\alpha=\hat{\Psi}\left(x_{1}+y\right)-\hat{\Psi}\left(x_{1}\right)$, se tiene

$$
y^{*}(y) \leq \hat{\Psi}\left(x_{1}+y\right)-\hat{\Psi}\left(x_{1}\right), \forall y \in X .
$$

Usando la definición de $\Psi$, la expresión anterior se transforma en

$$
y^{*}(x)-y^{*}\left(x_{1}\right) \leq f(x)-f\left(x_{1}\right)-x_{0}^{*}\left(x-x_{1}\right), \forall x \in C .
$$


Así pues, hemos obtenido que el funcional $x_{1}^{*}:=y^{*}+x_{0}^{* *}$ está en el conjunto $\partial f\left(x_{1}\right)$ y, en vista de (4), satisface

$$
\left\|x_{0}^{*}-x_{1}^{*}\right\|=\left\|y^{*}\right\| \leq \lambda .
$$

Este hecho, junto con (2), permite dar por concluida la demostración.

El resultado anterior puede también verse como un principio de optimización perturbada; a partir de él es sencillo de obtener el siguiente:

1.9 Corolario. Si $X$ es un espacio de Banach real, $C$ un subconjunto no vacío, convexo y cerrado del mismo y $f: C \longrightarrow \mathbf{R}$ una función inferiormente semicontinua, convexa y minorada; entonces, dados un número real positivo $\varepsilon$ y un elemento $x_{0}$ de $C$ tales que

$$
f\left(x_{0}\right) \leq \inf f(C)+\varepsilon
$$

existe un funcional $x^{*} \in X^{*}$, con $\left\|x^{*}\right\| \leq \sqrt{\varepsilon}$ y de forma que $f+x^{*}$ alcanza su mínimo en un punto $x_{1} \in C$ que verifica

$$
\left\|x_{0}-x_{1}\right\| \leq \sqrt{\varepsilon} \text {. }
$$

Demostración. Podemos suponer sin pérdida de generalidad que inf $f(C)=0$. Por hipótesis, $f\left(x_{0}\right) \leq \varepsilon$, luego el funcional $0 \in \partial_{\varepsilon} f\left(x_{0}\right)$; entonces, el Teorema de Bronsted-Rockafellar nos proporciona un vector $x_{1} \in C$ y un funcional $x_{1}^{*} \in \partial f\left(x_{1}\right)$ tales que

$$
\left\|x_{0}-x_{1}\right\| \leq \sqrt{\varepsilon} \text { y }\left\|x_{1}^{*}\right\| \leq \sqrt{\varepsilon} .
$$

Tomando $x^{*}:=-x_{1}^{*}$, la función $f+x^{*}$ alcanza el mínimo en $C$, ya que por ser $x_{1}^{*} \in \partial f\left(x_{1}\right)$ se tiene

$$
f\left(x_{1}\right)-x_{1}^{*}\left(x_{1}\right) \leq f(y)-x_{1}^{*}(y), \forall y \in C .
$$


1.10 Corolario. Sea $C$ un subconjunto no vacio, convexo y cerrado de wn espacio de Banach $X$ (real o complejo). Supongamos que $x_{0}^{*} \in X^{*} \backslash\{0\}$ es un funcional con parte real mayorada en $C y$ consideremos $0<\varepsilon<\left\|x_{0}^{*}\right\| y x_{0} \in C$ tales que

$$
\operatorname{Re} x_{0}^{*}\left(x_{0}\right)>\operatorname{Sup} \operatorname{Re} x_{0}^{*}(C)-\varepsilon^{2} .
$$

Entonces, existen un elemento $x_{1} \in C$ y un funcional $x_{1}^{*} \in X^{*} q u e$ soporta a $C$ en $x_{1}$ verificando

$$
\left\|x_{0}-x_{1}\right\| \leq \varepsilon \quad \text { y }\left\|x_{0}^{*}-x_{i}^{*}\right\| \leq \varepsilon .
$$

Demostración. El caso complejo se reduce trivialmente al real. Sean pues $X$ un espacio de Banach real y $x_{0}^{*}, \varepsilon y x_{0}$ como en el enunciado. Definimos

$$
f: C \longrightarrow \mathbb{R}
$$

por

$$
f(x)=0, \forall x \in C \text {. }
$$

Entonces $x_{0}^{*} \in \partial_{\varepsilon^{2}} f\left(x_{0}\right)$ y el Teorema de Bronsted-Rockafellar proporciona un elemento $x_{1}$ de $C$ y un funcional $x_{i}^{*} \in \partial f\left(x_{1}\right)$, esto es, $x_{1}^{*}$ es un funcional de soporte de $C$ en $x_{1}$, tal que

$$
\left\|x_{0}-x_{1}\right\| \leq \varepsilon \text { y }\left\|x_{0}^{*}-x_{1}^{*}\right\| \leq \varepsilon
$$

(nótese que $x_{1}^{*} \neq 0$, puesto que $\left.\left\|x_{0}^{*}-x_{1}^{*}\right\| \leq \varepsilon<\left\|x_{0}^{*}\right\|\right)$.

Enunciemos ya el teorema estrella de este capítulo que no es más que una consecuencia directa del corolario anterior y el Teorema de separación de conjuntos convexos. Se trata nuevamente de un principio de optimización perturbada. 
1.11 Corolario (Teorema de Bishop y Phelps [5, Theorem 1, Corollary 4]). Sea $C$ un subconjunto no vacio, convexo y cerrado de un espacio de Banach $X$ (real o complejo). Se verifica entonces

(i) Los puntos de soporte forman un conjunto denso en la frontera de $C$.

(ii) El conjunto de los funcionales de soporte de $C$ es denso en el cono formado por los funcionales de parte real mayorada en $C$.

Demostración. El apartado (ii) es obvio a partir del corolario anterior. Para probar (i), sean $x_{0} \in F r(C)$ y $0<\varepsilon<1$. Elegimos $y \in X \backslash C$ verificando $\left\|x_{0}-y\right\|<\varepsilon^{2}$. El Teorema de separación de convexos nos da un funcional $x_{0}^{*}$, que podemos suponer en la esfera unidad, tal que

$$
\operatorname{Sup} \operatorname{Re} x_{0}^{*}(C)<\operatorname{Re} x_{0}^{*}(y)
$$

y de ahí,

$$
\text { Sup } \operatorname{Re} x_{0}^{*}(C)<\operatorname{Re} x_{0}^{*}\left(y-x_{0}\right)+\operatorname{Re} x_{0}^{*}\left(x_{0}\right)<\varepsilon^{2}+\operatorname{Re} x_{0}^{*}\left(x_{0}\right) .
$$

El Corolario 1.10 asegura la existencia de un punto de soporte $x_{1}$ de $C^{\prime}$ tal que

$$
\left\|x_{0}-x_{1}\right\| \leq
$$

Nótese que como consecuencia del resultado anterior, obtenemos que en cualquier espacio de Banach, el conjunto de los funcionales que alcanzan su norma es denso en el dual.

Si particularizamos la mejora que hemos presentado del Teorema de Bishop-Phelps (Corolario 1.10) al caso de la bola unidad, se obtiene el siguiente resultado probado por Bollobás en 1.970: 
1.12 Corolario (Teorema de Bishop-Phelps-Bollobás [6]). Sea $X$ un espacio de Banach. Supongamos que $x_{0}^{*} \in S_{X *,} \varepsilon>0$ y $x_{0} \in S_{X}$ verifican

$$
\operatorname{Re} x_{0}^{*}\left(x_{0}\right)>1-\varepsilon^{2} \text {. }
$$

Entonces, existen $x_{1} \in S_{X}$ y $x_{1}^{*} \in S_{X}$. tales que $x_{1}^{*}\left(x_{1}\right)=1$,

$$
\left\|x_{0}-x_{1}\right\| \leq \varepsilon y\left\|x_{0}^{*}-x_{1}^{*}\right\| \leq \varepsilon .
$$

En el Teorema de Bishop-Phelps la complitud es esencial: con respecto a la afirmación (i) Klee [26] encontró un subconjunto no vacío, convexo, y cerrado de un subespacio denso de un espacio de Hilbert sin puntos de soporte. En el apartado (ii) también es necesaria:

1.13 Proposición (Bishop y Phelps [5, Theorem 3]). Sea $X$ un espacio normado no completo. Entonces, existe un subconjunto $C$ no vacio, convexo, cerrado, acotado y con interior no vacio tal que el conjunto de los funcionales de soporte de $C$ no es denso en $X^{*}$.

Demostración. Podemos suponer, sin pérdida de generalidad, que $X$ es un espacio vectorial real. Notemos por $\hat{X}$ la completación de $X$ e identifiquemos de forma natural los duales de ambos espacios. Elegimos un vector $x \in \hat{X} \backslash X$ de norma uno. El Teorema de Hahn-Banach proporciona un funcional $x^{*}$ en $S_{\hat{X}}$. tal que $x^{*}(x)=1$. Consideremos los conjuntos $D$ y $C^{\prime}$ definidos por

$$
D=B_{\hat{X}} \cap \operatorname{ker}\left(x^{*}\right)
$$

y

$$
C^{\prime}=\operatorname{co}(D \cup\{x\})
$$


Es fácil comprobar que $C^{\prime}$ es cerrado y además, tiene interior no vacío ya que si $z \in \hat{X}$ y $\|z-x / 2\|<1 / 6$, tomando $t=x^{*}(z)$, es claro que

$$
1-t=x^{*}(x-z) \geq x^{*}(x / 2)-\left|x^{*}(x / 2-z)\right| \geq \frac{1}{3}
$$

y

$$
|t-1 / 2|=\left|x^{*}(z-x / 2)\right|<\frac{1}{6}
$$

en particular, $0<t<1$. El elemento $y=\frac{z-t x}{1-t}$ está en ker $x^{*}$ y en $B_{\hat{X}}$ en vista de que

$$
\|y\| \leq \frac{1}{1-t}(\|z-x / 2\|+\|x / 2-t x\|) \leq 1
$$

por tanto, $z=t x+(1-t) y \in C^{\prime}$.

Definimos ya $C$ como

$$
C=C^{\prime} \cap X
$$

Entonces $\mathrm{C}$ es no vacío, convexo, cerrado y tiene interior no vacío. Veamos que si $y^{*}$ es un funcional de $X^{*}$ que soporta a $C$, entonces $\left\|x^{*}-y^{*}\right\| \geq 1 / 2$. Sea pues $z \in C$ tal que $y^{*}(z)=\sup y^{*}(C)$; existen entonces un escalar $\lambda \in[0,1[y$ un elemento $y \in D$ tales que $z=\lambda x+(1-\lambda) y$. Como $C^{\prime}$ es convexo y tiene interior no vacío, $\sup y^{*}(C)=\sup y^{*}\left(C^{\prime}\right)$, luego

$$
y^{*}(x) \leq y^{*}(z)=(1-\lambda) y^{*}(y-x)+y^{*}(x)
$$

de donde

$$
y^{*}(y-x) \geq 0
$$

Pero

$$
1=x^{*}(x-y) \leq\|x-y\| \leq\|x\|+\|y\| \leq 2
$$


y por tanto,

$$
1 \leq\left(y^{*}-x^{*}\right)(y-x) \leq\left\|x^{*}-y^{*}\right\|\|x-y\| \leq 2\left\|x^{*}-y^{*}\right\|
$$

que es lo que queriamos probar.

En vista de que en el Teorema de Bishop-Phelps la complitud del espacio es indispensable, la naturaleza del problema general de la densidad de los operadores que alcanzan la norma nos obliga a considerar en el resto de la memoria únicamente espacios de Banach.

En un espacio de Banach complejo, es evidente que los puntos y funcionales de soporte de un subconjunto dependen únicamente dei espacio real subyacente. Por tanto, si bien formalmente el erinciado del Teorema de Bishop-Phelps (Corolario 1.11) es válido en caso real o complejo, hubiese bastado formularlo sólo para espacios de Banach reales. Sin duda alguna, una versión de dicho teorema que involucre el hecho de que el espacio vectorial sea complejo, debería poderse enunciar en términos de funcionales y puntos módulo-soporte de un subconjunto, conceptos que ahora presentamos.

1.14 Definición. Sea $C$ un subconjunto no vacío, convexo y cerrado de un espacio normado $X$. Un vector $x_{0} \in C$ se dice punto módulo-soporte de $C$ si existe un funcional $x^{*} \in X^{*} \backslash\{0\}$ cuyo módulo alcanza en $x_{0}$ su supremo en $C$; a $x^{*}$ se le llama funcional módulo-soporte de $C$ (en $x_{0}$ ).

A la luz del Teorema de Bishop-Phelps es natural plantearse la siguiente cuestión: 
1.15 Problema (Phelps [31]). Dado $C$ un subconjunto no vacio, convexo y cerrado de un espacio de Banach complejo,

(i) ¿Es denso en la frontera de $C$ el conjunto de los puntos módulosoporte?

(ii) ¿Es el conjunto de los funcionales módulo-soporte de $C$ denso en el subespacio formado por los funcionales cuyo módulo está acotado en $C$ ?

La pregunta (i) es resuelta negativamente por Phelps. Basta notar que, tomando $C=\operatorname{co}\left(B_{\mathrm{C}} \cup\{2\}\right) \subseteq \mathbb{C}$, no hay más puntos módulo-soporte que $x_{0}=2$.

Para reformular el apartado (ii) del problema, Phelps impone la hipótesis adicional de acotación para $C$. En este caso, $\mathbb{D} C$ es cerrado, donde $\mathbf{D}=\{\lambda \in \mathbb{C}:|\lambda| \leq 1\}$, y la pregunta planteada en (ii) se pone en equivalencia de forma inmediata con esta otra:

1.16 Problema. ¿Es denso en $\left(X_{\mathbf{R}}\right)^{*}$ el conjunto de los funcionales de $\left(X_{\mathbf{R}}\right)^{*}$ de soporte de $\mathbb{D} C$ ? ( $X_{\mathbf{R}}$ representa el espacio real subyacente a $X)$.

Así pues, si $C$ es un subconjunto no vacío, convexo, cerrado y acotado de $X$ tal que $D C$ es convexo, el Teorema de Bishop-Phelps nos da respuesta afirmativa. Como los funcionales que soportan a $\mathbf{D C}$ coinciden con los que soportan a $\operatorname{co}(\mathbb{D} C)$, si $C$ es un subconjunto no vacío, convexo, cerrado y acotado de modo que $\operatorname{co}(\mathbb{D} C)$ es cerrado, el Problema 
1.15.(ii) se responde afirmativamente. No siempre se tiene esta situación; el conjunto

$$
C=\left\{x \in c_{0}: x \geq 0, \sum_{n=1}^{\infty} x(n) \leq 1\right\}
$$

es obviamente convexo, cerrado y acotado. En este caso, $\operatorname{co}(\mathbb{D C})$ no es cerrado y, sin embargo, el Problema 1.15.(ii) tiene respuesta afirmativa, ya que $\overline{c o(D C)}=B_{l_{1}}, \mathrm{y}$, por tanto, cualquier funcional módulo-soporte de $\overline{c o(D C)}$ lo es también de $C$.

Si $X$ es un espacio de Banach real, R.R. Phelps prueba que la pregunta (ii) del Problema 1.15 tiene respuesta afirmativa ([31, Proposition 7]), mientras que (i) es claramente falso; tómese $C=\operatorname{co}\left(B_{\mathbf{R}^{2}} \cup\{2\}\right) \subseteq \mathbb{R}^{2}$ y -1 no está en el cierre de los puntos módulo-soporte de $\operatorname{Fr}(C)$.

Para finalizar el capítulo maticemos lo siguiente: en la literatura (y nosotros también a partir de este momento) por el Teorema de BishopPhelps suele entenderse el resultado primero de estos autores [4] que afirma la densidad de los funcionales que alcanzan su norma. 


\section{Capítulo 2}

\section{El Teorema de Lindenstrauss-Zizler.}

Ya en su primer artículo ([4]), E. Bishop y R.R. Phelps sugieren el problema de ia posible validez de su teorema cambiando el cuerpo base por un espacio de Banach. J. Lindenstrauss probó que la respuesta a este problema no es siempre afirmativa. Para concretarlo, fijaremos la siguiente notación. En lo sucesivo, si $X$ e $Y$ son espacios de Banach, $L(X, Y)$ representará el espacio de Banach de los operadores (lineales y continuos) de $X$ en $Y$ dotado de su norma usual, esto es,

$$
\|T\|=\sup \left\{\|T x\|: x \in B_{X}\right\} \quad(T \in L(X, Y)) .
$$

Para $T \in L(X, Y), T^{*}$ denotará el operador adjunto de $T$. Diremos que un operador $T \in L(X, Y)$ alcanza su norma si existe un elemento $x_{0} \in S_{X}$ tal que $\left\|T x_{0}\right\|=\|T\|$. Notaremos por $N A(X, Y)$ el conjunto de los operadores de $X$ en $Y$ que alcanzan su norma. 
El problema al que hernos hecho alusión se enuncia en estos términos:

2.1 Problema de Bishop-Phelps. Si $X$ e $Y$ son espacios de Banach, ipuede asegurarse que el conjunto $N A(X, Y)$ es denso en $L(X, Y)$ ?

Si $X$ es finito dimensional, la compacidad de $B_{X}$ nos asegura que para cualquier espacio de Banach $Y$, se verifica $N A(X, Y)=L(X, Y)$. El Teorema de Bishop-Phelps nos dice que $\overline{N A(X, \mathbb{K})}=L(X, \mathbb{K})$, para cualquier espacio de Banach $X$.

Sin embargo, el problema de Bishop-Phelps no tiene una respuesta afirmativa en general, como pone de manifiesto la siguiente observación debida a J. Lindenstrauss ([27]).

2.2 Lema. Sean $X$ e $Y$ dos espacios de Banach, tal que $Y$ es estrictamente convexo, y $T \in L(X, Y)$ un operador inyectivo. Entonces, si $T$ alcanza su norma, existe un punto extremo $x_{0}$ en $B_{X}$ de forma que $\left\|T x_{0}\right\|=\|T\|$.

Demostración. Sea $x_{0} \in S_{X}$ tal que $\left\|T x_{0}\right\|=\|T\|$. Si $x_{1}$ y $x_{2}$ son dos vectores de la esfera unidad de $X$ satisfaciendo la igualdad

$$
x_{0}=\frac{1}{2}\left(x_{1}+x_{2}\right)
$$

entonces $T x_{0}=\frac{1}{2}\left(T x_{1}+T x_{2}\right)$ y como $T$ alcanza su norma en $x_{0}$, se tiene

$$
\left\|T x_{0}\right\|=\left\|T x_{1}\right\|=\left\|T x_{2}\right\| .
$$


La convexidad estricta de $Y$ nos asegura que $T x_{1}=T x_{2}$, y la inyectividad de $T$ que $x_{1}=x_{2}$, por lo que $x_{0}$ es un punto extremo de $B_{X}$.

Así pues, si disponemos de dos espacios de Banach isomorfos $X$ e $Y$, taies que $B_{X}$ carece de puntos extremos e $Y$ es estrictamente convexo, entonces $\overline{N A(X, Y)} \neq L(X, Y)$. El siguiente resultado nos garantiza la existencia de un espacio $Y$ que verifique lo anterior tomando $X=c_{0}$.

2.3 Lema. Sean $X$ e $Y$ dos espacios de Banach, con $Y$ estrictamente convexo y supongamos que $T \in L(X, Y)$ es un operador inyectivo. Entonces, la norma en $X$ definida por

$$
\|x\|:=\|x\|+\|T x\| \quad(x \in X)
$$

es estrictamente convexa.

Demostración. Sean $x_{1}$ y $x_{2}$ dos vectores de $X$ tales que

$$
\left\|x_{1}\right\|=\left\|x_{2}\right\|=1, \quad \text { y }\left\|x_{1}+x_{2}\right\|=2 .
$$

Entonces, usando la definición de $\|$. $\|$ y la desigualdad triangular llegamos a que

$$
\left\|T x_{1}+T x_{2}\right\|=\left\|T x_{1}\right\|+\left\|T x_{2}\right\| .
$$

Por ser $Y$ estrictamente convexo, encontramos un número real $\rho \geq 0$ tal que

$$
T x_{1}=\rho T x_{2}
$$

y puesto que $T$ es inyectivo ha de ser $x_{1}=\rho x_{2}$. La condición (1) nos da inmediatamente $\rho=1$, luego $x_{1}=x_{2}$. 
Tomando en el lema anterior $X=c_{0}, Y=l_{2}$ y $T: c_{0} \longrightarrow l_{2}$ definido por

$$
(T x)(n):=\frac{x(n)}{2^{n / 2}} \quad\left(n \in \mathbb{N}, x \in c_{0}\right),
$$

donde $x(n)$ es el n-ésimo término de la sucesión $x$, obtenemos en $c_{0}$ una norma $\|\cdot \mid\|$ estrictamente convexa y equivalente a la usual $\|\cdot\|$ :

$$
\|x\|=\|x\|+\left(\sum_{n=1}^{\infty} \frac{|x(n)|^{2}}{2^{n}}\right)^{1 / 2} \quad\left(x \in c_{0}\right) .
$$

Entonces $\left(c_{0},\|\cdot\|\right)$ y $\left(c_{0},\|\cdot\| \|\right)$ son dos espacios de Banach isomorfos, el primero de los cuales tiene una bola desprovista de puntos extremos y el segundo estrictamente convexo, por lo que, en vista del Lema 2.2, el conjunto $N A\left(\left(c_{0},\|\cdot\|\right),\left(c_{0},\|\cdot\| \|\right)\right)$ no es denso en el correspondiente espacio de operadores.

El caso de $X=c_{0}$ permite afinar aún más el resultado anterior:

2.4 Proposición ([27, Proposition 4]). Consideremos un espacio de Banach $Y$ estrictamente convexo $y$ un operador $T \in N A\left(c_{0}, Y\right)$. Entonces, existe un número natural $\nu$ tal que

$$
T e_{n}=0, \quad \text { si } n \geq \nu,
$$

donde $\left\{e_{n}\right\}_{n \in \mathbb{N}}$ es la base usual de $c_{0}$. En particular, $T$ es un operador de rango finito y por tanto, si existe un operador no compacto de $c_{0}$ en $Y$, entonces $N A\left(c_{0}, Y\right)$ no es denso en $L\left(c_{0}, Y\right)$.

Demostración. Sean $T$ un operador de $c_{0}$ en $Y$ y $x \in S_{c_{0}}$ tales que $\|T x\|=\|T\|$. Por ser $x$ convergente a cero, existe un natural $\nu$ verificando

$$
\left\|x \pm \frac{1}{2} e_{n}\right\|=1, \quad \text { si } n \geq \nu
$$


de donde

$$
\left\|T x \pm \frac{1}{2} T e_{n}\right\| \leq\|T x\|
$$

y la convexidad estricta de $Y$ nos asegura que

$$
T e_{n}=0, \quad \forall n \geq \nu .
$$

Si bien no siempre está garantizada la densidad de $N A(X, Y)(X$ e $Y$ espacios de Banach arbitrarios) en $L(X, Y)$, a continuación vamos a definir otro subconjunto de dicho espacio, determinado por una propiedad similar. Previamente observemos que, como consecuencia del Teorema de Hahn-Banach, si un operador $T \in L(X, Y)$ alcanza su norma, también $T^{*}$ lo hace. Definimos los subconjuntos de $L(X, Y)$

$$
\begin{aligned}
& N A_{1}(X, Y):=\left\{T \in L(X, Y): T^{*} \in N A\left(Y^{*}, X^{*}\right)\right\} \text { y } \\
& N A_{2}(X, Y):=\left\{T \in L(X, Y): T^{* *} \in N A\left(X^{* *}, Y^{* *}\right)\right\} .
\end{aligned}
$$

El comentario anterior se traduce en la siguiente cadena de inclusiones:

$$
N A(X, Y) \subseteq N A_{1}(X, Y) \subseteq N A_{2}(X, Y) .
$$

Las inclusiones anteriores pueden ser estrictas como prueba el siguiente ejemplo: considérese el operador

$$
T: c_{0} \longrightarrow c_{0}
$$

dado por

$$
T x=\sum_{n=1}^{\infty}\left(1-\frac{1}{n}\right) x(n) e_{n}, \quad\left(x \in c_{0}\right) .
$$

Es inmediato que, usando la identificación canónica de $c_{0}^{*} \equiv l_{1}$, el operador $T^{*}$ adopta la expresión

$$
T^{*} x=\sum_{n=1}^{\infty}\left(1-\frac{1}{n}\right) x(n) e_{n}, \quad\left(x \in l_{1}\right)
$$


Claramente $T^{*}$ no alcanza su norma, pero $T^{* *} \in L\left(l_{\infty}\right)$ sí la alcanza en $u \in l_{\infty}$, siendo $u(n)=1, \forall n \in \mathbb{N}$.

J. Lindenstrauss ([27]) probó que $N A_{2}(X, Y)$ es siempre denso en $L(X, Y)$ y V. Zizler $([40])$, basándose en el trabajo de aquél, que, de hecho, $N A_{1}(X, Y)$ también lo es.

Previamente a la prueba del resultado, caractericemos de forma cómoda los elementos de $N A_{1}(X, Y)$.

2.5 Lema ([40, Lemma 1]). Dados dos espacios de Banach $X$ e $Y y$ un operador $T \in L(X, Y)$, es condición necesaria y suficiente para que $T \in N A_{1}(X, Y)$, que existan sucesiones $\left\{x_{n}\right\}_{n \in \mathbb{N}}$ en $S_{X}$ e $\left\{y_{n}^{*}\right\}_{n \in \mathbb{N}}$ en $S_{Y}$ tales que

$$
\left|y_{n}^{*}\left(T x_{m}\right)\right| \geq\|T\|-1 ; m, \quad \forall n, m \in \mathbb{N}, m<n
$$

Demostración. La condición es claramente necesaria: si $T^{*}$ alcanza la norma en $y^{*} \in S_{Y} \cdot$, tomamos $y_{n}^{*}=y^{*}$ para cada natural $n$ y como $\left\|T^{*} y^{*}\right\|=\|T\|$, basta elegir para cada $m, x_{m} \in S_{X}$ tal que

$$
\left|\left(T^{*} y^{*}\right)\left(x_{m}\right)\right| \geq\|T\|-1 / m .
$$

También es suficiente: puesto que $B_{Y} \cdot$ es $\omega^{*}$-compacta, sea $y^{*} \in B_{Y} \cdot$ un valor adherente de la sucesión $\left\{y_{n}^{*}\right\}_{n \in N}$. Por tanto, de la relación que liga a $T$ y las dos sucesiones, se sigue que

$$
\left\|T^{*} y^{*}\right\| \geq\left|y^{*}\left(T x_{m}\right)\right| \geq\|T\|-1 / m, \forall m \in \mathbb{N}
$$

de donde

$$
\left\|T^{*} y^{*}\right\|=\|T\|
$$


2.6 Teorema ([40, Proposition 4]). Para cualesquiera espacios de Banach $X$ e $Y$, el conjunto $N A_{Y}(X, Y)$ es denso en el espacio $L(X, Y)$.

Demostración. Sean $T$ un operador de $L(X, Y)$ con $\|T\|=1$ y $\varepsilon$ un número real tal que $0<\varepsilon<1 / 3$. A partir de $T$ vamos a construir un operador $S$ cuyo adjunto alcanza la norma y que verifica

$$
\|T-S\|<\varepsilon .
$$

Sea $\left\{\varepsilon_{n}\right\}_{n \in \mathbb{N}}$ una sucesión decreciente de números reales positivos satisfaciendo las siguientes tres condiciones:

$$
\sum_{i=1}^{\infty} \varepsilon_{i}<\varepsilon / 2, \sum_{i=n+1}^{\infty} \varepsilon_{i}<\varepsilon_{n}^{2} / 2, \varepsilon_{n}<\frac{1}{10(n+1)}, \quad \forall n \in \mathbb{N}
$$

Definimos por recurrencia una sucesión de operadores $\left\{T_{n}\right\}_{n \in \mathbb{N}}$ como sigue. Tomamos

$$
T_{1}=T
$$

y para cada natural $n$, supuesto construido $T_{n} \in L(X, Y)$, elegimos $y_{n}^{*} \in S_{Y} \cdot$ y $x_{n} \in S_{X}$ de modo que

$$
\left\|T_{n}^{*} y_{n}^{*}\right\| \geq\left\|\tilde{i}_{n}\right\|-\varepsilon_{n}^{2}
$$

e

$$
y_{n}^{*}\left(T_{n} x_{n}\right)=\left|y_{n}^{*}\left(T_{n} x_{n}\right)\right| \geq\left\|T_{n}^{*} y_{n}^{*}\right\|-\varepsilon_{n}^{2}
$$

Se define

$$
T_{n+1} x=T_{n} x+\varepsilon_{n} y_{n}^{*}\left(T_{n} x\right) T_{n} x_{n} \quad(x \in X) .
$$

Es fácil comprobar por inducción que para cada natural $n$ se verifica

$$
\left\|T_{n+1}\right\| \leq 1+\sum_{i=1}^{n} 2 \varepsilon_{i} \leq 4 / 3
$$


de donde

$$
\left\|T_{n+1}-T_{m}\right\| \leq \sum_{i=m}^{n} 2 \varepsilon_{i}, \text { si } m \leq n .
$$

Por tanto, la sucesión $\left\{T_{n}\right\}_{n \in N}$ converge en la topología de la norma hacia un operador $S \in L(X, Y)$ que, a la luz de (4), satisface

$$
\left\|T_{m}-S\right\| \leq 2 \sum_{i=m}^{\infty} \varepsilon_{i}<\varepsilon_{m-1}, \quad \forall m \in \mathbb{N}
$$

y, en particular, $\|S-T\|<\varepsilon . S$ es el operador buscado, ya que como probamos a continuación, $S^{*}$ alcanza la norma. Es claro que

$$
T_{n+1}^{*} y^{*}=T_{n}^{*} y^{*}+\varepsilon_{n}\left[\left(T_{n}^{*} y^{*}\right)\left(x_{n}\right)\right] T_{n}^{*} y_{n}^{*} \quad\left(y^{*} \in Y^{*}\right),
$$

de donde, usando (1), (2), (3) y la mayoración de $\left\|T_{n}\right\|$, obtenemos

$$
\begin{gathered}
\left\|T_{n+1}\right\| \geq\left\|T_{n+1}^{*} y_{n}^{*}\right\|=\left\|T_{n}^{*} y_{n}^{*}\right\|\left(1+\varepsilon_{n}\left(T_{n}^{*} y_{n}^{*}\right)\left(x_{n}\right)\right) \geq \\
\geq\left(\left\|T_{n}\right\|-\varepsilon_{n}^{2}\right)\left(1+\varepsilon_{n}\left\|T_{n}\right\|-2 \varepsilon_{n}^{3}\right) \geq \\
\geq\left\|T_{n}\right\|+\varepsilon_{n}\left\|T_{n}\right\|^{2}-\varepsilon_{n}^{2}\left(1+3 \varepsilon_{n}\left\|T_{n}\right\|\right) \geq \\
\geq\left\|T_{n}\right\|+\varepsilon_{n}\left\|T_{n}\right\|^{2}-5 \varepsilon_{n}^{2} .
\end{gathered}
$$

Como consecuencia, la sucesión $\left\{\left\|T_{n}\right\|\right\}_{n \in N}$ es creciente. Este hecho, junto con (2) y (4), nos permite deducir, para $m<n$,

$$
\begin{gathered}
\left\|T_{m+1}^{*} y_{n}^{*}\right\| \geq\left\|T_{n}^{*} y_{n}^{*}\right\|-\left\|T_{n}-T_{m+1}\right\| \geq \\
\geq\left\|T_{n}\right\|-\varepsilon_{n}^{2}-\varepsilon_{m}^{2} \geq\left\|T_{n}\right\|-2 \varepsilon_{m}^{2} \geq\left\|T_{m+1}\right\|-2 \varepsilon_{m}^{2} .
\end{gathered}
$$

Haciendo uso de (6) y (7) conseguimos, si $m<n$,

$$
\begin{aligned}
& \varepsilon_{m}\left|\left(T_{m}^{*} y_{n}^{*}\right)\left(x_{m}\right)\right|\left\|T_{m}\right\|+\left\|T_{m}\right\| \geq\left\|T_{m+1}^{*} y_{n}^{*}\right\| \geq \\
& \geq\left\|T_{m+1}\right\|-2 \varepsilon_{m}^{2} \geq\left\|T_{m}\right\|+\varepsilon_{m}\left\|T_{m}\right\|^{2}-7 \varepsilon_{m}^{2}
\end{aligned}
$$


y basta dividir por $\varepsilon_{m}\left\|T_{m}\right\|$ para cener

$$
\left|y_{n}^{*}\left(T_{m} x_{m}\right)\right| \geq\left\|T_{m}\right\|-\frac{7 \varepsilon_{m}^{2}}{\varepsilon_{m}\left\|T_{m}\right\|} \geq\left\|T_{m}\right\|-7 \varepsilon_{m} .
$$

Para aplicar el Lema 2.5, cambiamos en la desigualdad anterior $T_{m}$ por $S$, usando la estimación de la norma dada en (5); así, tenemos

$$
\begin{gathered}
\left|y_{n}^{*}\left(S x_{m}\right)\right| \geq\left|y_{n}^{*}\left(T_{m} x_{m}\right)\right|-\left|y_{n}^{*}\left(T_{m} x_{m}\right)-y_{n}^{*}\left(S x_{m}\right)\right| \geq \\
\geq\left\|T_{m}\right\|-7 \varepsilon_{m}-\left\|T_{m}-S\right\| \geq\left\|T_{m}\right\|-7 \varepsilon_{m}-\varepsilon_{m-1}^{2} \geq \\
\geq\|S\|-8 \varepsilon_{m}-2 \varepsilon_{m-1}^{2} \geq\|S\|-1 / m, \quad \forall m<n .
\end{gathered}
$$

El lema anterior resuelve entonces la prueba.

La demostración que hemos ofrecido presenta una pequeña modifcación con respecto a la de Zizler: él trabaja en el espacio de operadores entre dos espacios de Banach duales y partiendo de un operador $\omega^{*}$-continuo (en nuestro caso $T^{*} \in L\left(Y^{*}, X^{*}\right)$ ) obtiene otro próximo que alcanza su norma; para terminar ha de probar que el operador que obtiene es $\omega^{*}$-continuo, lo que para nosotros es evidente porque es un operador adjunto.

Señalemos también que hay un resultado de R.A. Poliquin y V.E. Zizler ([33, Theorem 1]) que permite refinar el Teorema 2.6: podemos obtener, de hecho, un operador cuyo adjunto alcanza su norma próximo a un operador dado de forma que la diferencia de ambos operadores sea de rango uno (usando el unismo tipo de construcción que aparece en el Corolario 4.12 de esta memoria).

Por último observemos que si $X$ es un espacio de Banach reflexivo, entonces $N A(X, Y)=N A_{2}(X, Y)$, para cualquier espacio de Banach $Y$. Obtenernos así el siguiente resultado, debido a J. Lindenstrauss: 
2.7 Corolario ([27]). Si $X$ es un espacio de Banach reflexivo, entonces para cualquier espacio de Banach $Y$, el conjunto $N A(X, Y)$ es denso en el espacio $L(X, Y)$. 


\section{Capítulo 3}

\section{Las propiedades " $A$ " y " $B$ " de Lindenstrauss.}

El propósito del presente capítulo es ofrecer un tratamiento parcial del problema de Bishop-Phelps (Problema 2.1) con objeto de facilitar el análisis del mismo. Para tal fin, usaremos las propiedades $A$ y $B$ definidas por J. Lindenstrauss. Probaremos que dos condiciones de naturaleza geométrica, las propiedades $\alpha$ y $\beta$, son suficientes para que el espacio verifique $A$ y $B$ respectivamente. La ventaja de estas propiedades radica en que se definen de manera intrínseca, es decir, aparentemente no involucran el conjunto de operadores que alcanzan la norma y son muy generales desde el punto de vista isomórfico: J.R. Partington demosiró que todo espacio de Banach puede ser renormado equivalentemente con la propiedad $\beta$ y W. Schachermayer que todo espacio de Banach débilmente compactamente generado es isomorfo a otro espacio de Banach satisfaciendo la propiedad $\alpha$. 


\section{A. El Teorema de renormación de Partington.}

Vamos a concretar las propiedades que, como ya se ha dicho, harán más abordable el problema de Bishop-Phelps:

3.1 Definición ([27]). Un espacio de Banach $X$ (resp. $Y$ ) verifica la propiedad $A$ (resp. $B$ ) si el conjunto $N A(X, Y)$ es denso en el espacio $L(X, Y)$, para cualquier espacio de Banach $Y$ (resp. $X)$.

Todo espacio de Banach reflexivo tiene la propiedad $A$ (Corolario 2.7) y en virtud del Teorema de Bishop-Phelps el cuerpo cumple la $B$. Además, como se puso de manifiesto en el Capítulo II, $c_{0}$ no verifica la propiedad $A$.

3.2 Definición ([34, Definition 1.2.(b)]). Se dice que un espacio de Banach $Y$ verifica la propiedad $\beta$ si existen un conjunto $I$, un subconjunto $\left\{\left(y_{i}, y_{i}^{*}\right): i \in I\right\}$ de $S_{Y} \times S_{Y} \cdot$ y un número real $\lambda, 0 \leq \lambda<1$, satisfaciendo las tres condiciones siguientes:

(a) $y_{i}^{*}\left(y_{i}\right)=1, \forall i \in I$,

(b) $\left|y_{i}^{*}\left(y_{j}\right)\right| \leq \lambda, \forall i, j \in I, i \neq j$,

(c) $\|y\|=\sup \left\{\left|y_{i}^{*}(y)\right|: i \in I\right\}, \forall y \in Y$.

Si $Y$ es un espacio de Banach con la propiedad $\beta, X$ es otro espacio de Banach arbitrario y $T$ es un elemento de $L(X, Y)$, usando el apartado 
(c) de la definición anterior, es inmediato que la norma de $T$ viene dada por

$$
\|T\|=\sup \left\{\left\|T^{*} y_{i}^{*}\right\|: i \in I\right\} .
$$

3.3 Ejemplos. (i) $l_{\infty}, c_{0}$ y $c$ tienen la propiedad $\beta$. Para el espacio $l_{\infty}$ basta tomar $I=\mathbb{N}$ y los conjuntos $\left\{e_{n}: n \in \mathbb{N}\right\}$ y $\left\{e_{n}^{*}: n \in \mathbb{N}\right\}$, siendo

$$
\begin{gathered}
e_{n}(m)=0, \text { si } n \neq m \text { y } e_{n}(n)=1, \quad n, m \in \mathbb{N}, \\
e_{n}^{*}(x)=x(n), x \in l_{\infty}, n \in \mathbb{N}
\end{gathered}
$$

y $\lambda=0$. Como de hecho $e_{n} \in c_{0}$, para cada $n$, basta tomar como funcionales la restricción de los anteriores a $c_{0}$ y $c$ para comprobar que ambos espacios también verifican $\beta$.

(ii) Un espacio de Banach real de dimensión finita satisface la propiedad $\beta$ cuando, y sólo cuando, su bola unidad es un poliedro, i.e., intersección finita de semiespacios.

(iii) Si $\Omega$ es un espacio topológico con un subconjunto denso de puntos aislados, entonces el espacio de las funciones continuas y acotadas definidas en $\Omega, C_{b}(\Omega)$, con su norma usual, cumple la propiedad $\beta$. Para comprobarlo, notaremos por $\left\{\omega_{i}: i \in I\right\}$ al subconjunto de $\Omega$ denso de puntos aislados. Definimos entonces las funciones

$$
y_{i}(\omega)=\left\{\begin{array}{ll}
1, & \text { si } \omega=\omega_{i} \\
0, & \text { si } \omega \neq \omega_{i}
\end{array} \quad(\omega \in \Omega, i \in I)\right.
$$

y los funcionales de evaluación

$$
y_{i}^{*}(y)=y\left(\omega_{i}\right) \quad\left(y \in C_{b}(\Omega), i \in I\right) .
$$

Entonces, los conjuntos $\left\{y_{i}: i \in I\right\}$ y $\left\{y_{i}^{*}: i \in I\right\}$ satisfacen las condiciones de la propiedad $\beta$ para $\lambda=0$. 
3.4 Teorema ([27, Proposition 3]). Si un espacio de Banach $Y$ tiene la propiedad $\beta$, entonces también tiene la $B$. Es decir, si $X$ es cualquier espacio de Banach, entonces $N A(X, Y)$ es denso en $L(X, Y)$.

Demostración. Consideremos un operador $T \in L(X, Y)$ con $\|T\|=1$ y $\varepsilon \in \mathbb{R}^{+}$. Elegimos un número real positivo $0<\eta<\varepsilon(1-\lambda) / 2$ y un elemento $i_{0} \in I$ tal que

$$
1-\eta<\left\|T^{*} y_{i_{0}}^{*}\right\|
$$

El Teorema de Bishop-Phelps nos permite encontrar un funcional $x^{*} \in X^{*}$ que alcanza la norma de forma que

$$
\left\|x^{*}-T^{*} y_{i_{0}}^{*}\right\|<\eta \quad \text { y }\left\|x^{*}\right\|=\left\|T^{*} y_{i_{0}}^{*}\right\| .
$$

Definimos el elemento $S \in L(X, Y)$ por

$$
S x:=T x+\left[\left(1+\frac{\varepsilon}{2}\right) x^{*}(x)-\left(T^{*} y_{i_{0}}^{*}\right)(x)\right] y_{i_{0}}(x \in X) .
$$

Entonces, la elección de $\eta$ y $x^{*}$ hace que

$$
\|S-T\| \leq \frac{\varepsilon}{2}\left\|x^{*}\right\|+\left\|T^{*} y_{i_{0}}^{*}-x^{*}\right\|<\frac{\varepsilon}{2}+\eta<\varepsilon .
$$

Veamos que $S$ alcanza su norma; si $i \neq i_{0}$, entonces

$$
\begin{gathered}
\left\|S^{*} y_{i}^{*}\right\| \leq\|T\|+\left|y_{i}^{*}\left(y_{i_{0}}\right)\right|\left(\frac{\varepsilon}{2}\left\|x^{*}\right\|+\left\|T^{*} y_{i_{0}}^{*}-x^{*}\right\|\right) \leq \\
\leq 1+\lambda\left(\frac{\varepsilon}{2}+\eta\right),
\end{gathered}
$$

mientras que la elección de $x^{*}$ y de $y_{i_{0}}^{*}$ permite obtener

$$
\left\|S^{*} y_{i_{0}}^{*}\right\|=\left(1+\frac{\varepsilon}{2}\right)\left\|x^{*}\right\| \geq\left(1+\frac{\varepsilon}{2}\right)(1-\eta)
$$

De la elección de $\eta$ se sigue que

$$
1+\lambda\left(\frac{\varepsilon}{2}+\eta\right) \leq\left(1+\frac{\varepsilon}{2}\right)(1-\eta)
$$


Como $Y$ tiene la propiedad $\beta$, entorices

$$
\|S\|=\sup \left\{\left\|S^{*} y_{i}^{*}\right\|: i \in I\right\}
$$

y en vista de (1) y (2), tenemos

$$
\|S\|=\left\|S^{*} y_{i_{0}}^{*}\right\|
$$

y por alcanzar $S^{*} y_{i_{0}}^{*}$ la norma, ya que $S^{*} y_{i_{0}}^{*}=\left(1+\frac{\varepsilon}{2}\right) x^{*}, S$ también la alcanza.

El recíproco del resultado anterior no es cierto:

3.5 Ejemplo. A continuación ofrecemos un ejemplo que aparece en [3] de un espacio finito dimensional que no tiene la propiedad $\beta$ pero sí la $B$. De hecho, este espacio posee una debilitación de la propiedad $\beta$, llamada casi- $\beta$, que también implica la $B$ (véase [3]). Todos los espacios de Banach que hasta ahora se conocen con la propiedad $B$ tienen la mencionada propiedad casi- $\beta$. El espacio en cuestión es $Y=\mathbb{R}^{3}$ con la norma que hace que $B_{Y^{*}}=\operatorname{co}\left(A^{*} \cup\left(-A^{*}\right)\right)$, donde

$$
A^{*}=\left\{\left(\operatorname{sen} \frac{\pi}{2^{n}}, \cos \frac{\pi}{2^{n}}, 0\right): n \in \mathbb{N}\right\} \cup\{(0,1,1),(0,1,-1)\}
$$

Es claro que $Y$ no tiene la propiedad $\beta$ (su bola no es un poliedro). No obstante, sí verifica la propiedad $B$. Antes de comprobar esto, definimos la aplicación $\sigma: A^{*} \longrightarrow S_{Y}$ por

$$
\begin{gathered}
\sigma\left(\operatorname{sen} \frac{\pi}{2^{n}}, \cos \frac{\pi}{2^{n}}, 0\right)=\left(\operatorname{sen} \frac{\pi}{2^{n}}, \cos \frac{\pi}{2^{n}}, 0\right) \quad(n \in \mathbb{N}) \\
\sigma(0,1, \pm 1)=(0,1 / 2, \pm 1 / 2)
\end{gathered}
$$

y $\rho: A^{*} \longrightarrow \mathbb{R}_{0}^{+}$como

$$
\rho\left(\operatorname{sen} \frac{\pi}{2^{n}}, \cos \frac{\pi}{2^{n}}, 0\right)=\cos \frac{\pi}{2^{n}} \quad(n \in \mathbb{N})
$$


y

Es claro que

$$
\rho(0,0, \pm 1)=1 / 2
$$

$$
a^{*}\left(\sigma\left(a^{*}\right)\right)=1, \forall a^{*} \in A^{*}
$$

y

$$
\left|b^{*}\left(\sigma\left(a^{*}\right)\right)\right| \leq \rho\left(a^{*}\right)<1, \forall a^{*}, b^{*} \in A, a^{*} \neq b^{*} .
$$

Sea pues $X$ un espacio de Banach, $T \in L(X, Y)$ con $\|T\|=1$, y $\varepsilon>0$. $T^{*}: Y^{*} \rightarrow X^{*}$ alcanza su norma en un vector $a^{*} \in A^{*}$, esto es, $\left\|T^{*} a^{*}\right\|=1$. Dado $\eta>0$, el Teorema de Bishop-Phelps nos permite disponer de un funcional $x^{*} \in X^{*}$ que alcanza la norma y de forma que

$$
\left\|x^{*}-T^{*} a^{*}\right\|<\eta \text { y }\left\|x^{*}\right\|=1 \text {. }
$$

Definimos

$$
S x=T x+\left[\left(1+\frac{\varepsilon}{2}\right) x^{*}(x)-\left(T^{*} a^{*}\right)(x)\right] a \quad(x \in X),
$$

donde $a=\sigma\left(a^{*}\right)$. Claramente, haciendo el mismo tipo de estimaciones que en el Teorema 3.4, $\|T-S\|<\varepsilon$, y $S$ alcanza su norma en a (al menos, tomando $\eta$ suficientemente pequeño).

La propiedad $\beta$ no es estable por isomorfismos (piénsese en $\mathbf{R}^{2}$ ). Es máe, Partington probó en 1.982 que todo espacio de Banach es isomorfo a otro con la propiedad $\beta$ y, por tanto, con la $B$. En el curso de la demostración de este resultado haremos una construcción inductivotransfinita basada en la siguiente proposición.

Si $(\Gamma,<)$ es un conjunto bien ordenado, 1 representará su primer elemento. Además, dado $\gamma \in \Gamma$, notaremos por $\Gamma(\gamma)$ el subconjunto de $\Gamma$

$$
\Gamma(\gamma)=\{\delta \in \Gamma: \delta<\gamma, \delta \neq \gamma\} \text {. }
$$


3.6 Proposición (Principio de Inducción Transfinita). Sea $(\Gamma,<)$ un conjunto bien ordenado y $A$ un subconjunto suyo. Si $\gamma \in A$, para cada $\gamma \in \Gamma$ tal que $\Gamma(\gamma) \subseteq A$, entonces $\Gamma=A$.

Demostración. Si $\Gamma \neq A$, sea

$$
\gamma_{0}=\min \Gamma \backslash A .
$$

Entonces, $\Gamma\left(\gamma_{0}\right) \subseteq A$ y, por tanto, $\gamma_{0} \in A$, lo cual es absurdo.

El siguiente resultado nos permite construir, dados un conjunto bien ordenado $(\Gamma,<)$, un conjunto $\Lambda$ y un conjunto de proposiciones $\{P(\gamma): \gamma \in \Gamma\}$, para cada $\gamma \in \Gamma$ un elemento $\lambda_{\gamma} \in \Lambda$ satisfaciendo la proposición $P(\gamma)$ (véase una demostración en [12, Theorem II.5.2]).

3.7 Proposición (Principio de Construcción Transfinita). Sea $(\Gamma,<)$ un conjunto bien ordenado, $\Lambda$ un conjunto y $P: \Gamma \longrightarrow\{0,1\}^{\wedge}$ una aplicación. Supongamos que, dado $\gamma \in \Gamma \backslash\{1\}, y$ notando

$$
A_{\gamma}=\left\{f \in \Lambda^{\Gamma(\gamma)}: P(\delta)(f(\delta))=1, \quad \forall \delta \in \Gamma(\gamma)\right\},
$$

tenemos definida una aplicación

$$
\varphi_{\gamma}: A_{\gamma} \longrightarrow \Lambda
$$

tal que

$$
P(\gamma)\left(\varphi_{\gamma}(f)\right)=1, \quad \forall f \in A_{\gamma} .
$$

Entonces, si $\lambda_{1} \in \Lambda$ verifica $P(1)\left(\lambda_{1}\right)=1$, existe una única aplicación

$$
F: \Gamma \longrightarrow \Lambda
$$

de forma que

$$
F(1)=\lambda_{1}
$$


$y$

$$
F(\gamma)=\varphi_{\gamma}\left(F_{\mid \Gamma(\gamma)}\right), \quad \forall \gamma \in \Gamma \backslash\{1\}
$$

En particular,

$$
P(\gamma)(F(\gamma))=1, \quad \forall \gamma \in \Gamma
$$

En lo sucesivo, también será útil la siguiente

3.8 Definición. El carácter de densidad de un espacio de Banach $X$, que notaremos por dens $(X)$, es el menor ordinal $\nu$ verificando que existe un subconjunto $\left\{u_{\gamma}: \gamma<\nu\right\}$ de la esfera unidad denso en ésta.

3.9 Teorema ([29, Theorem 1]). Sea $(Y,\|\cdot\|)$ un espacio de Banach. Para cada $K>3$, existe una norma $\|$.$\| en Y$ tal que $(Y,\|\| \|)$ verifica la propiedad $\beta$ y

$$
\|y\| \leq\|y\| \leq K\|y\|, \quad \forall y \in Y .
$$

Demostración. Sea $\left\{u_{\gamma}: \gamma<\nu\right\}$ un subconjunto denso de $S_{Y}$, donde $\nu=\operatorname{dens}(Y)$. Para cada $\gamma<\nu$ elegimos un funcional $u_{\gamma}^{*} \in S_{Y} \cdot$ tal que

$$
u_{\gamma}^{*}\left(u_{\gamma}\right)=1 \text {. }
$$

A continuación cambiamos el conjunto $\left\{\left(u_{\gamma}, u_{\gamma}^{*}\right): \gamma<\nu\right\}$ por otro que satisfaga condiciones más próximas a las necesarias para la propiedad $\beta$. Sea $s$ un número real, con $2 /(K-1)<s<1$. En primer lugar, aplicamos el Principio de Construcción Transfinita, tomando $\Gamma=\nu$ y $\Lambda=S_{Y} \times S_{Y}$. Supongamos que, dado $\gamma \in \Gamma$, existe un subconjunto $\left\{\left(x_{\delta}, x_{\delta}^{*}\right): \delta<\gamma\right\} \subseteq \Lambda$ tal que para todo $\delta<\gamma$

$$
\operatorname{Re} x_{\delta}^{*}\left(x_{\delta}\right)=x_{\delta}^{*}\left(x_{\delta}\right)>s
$$


y

$$
x_{\delta}^{*}\left(x_{\alpha}\right)=x_{\delta}^{*}\left(u_{\alpha}\right)=0, \text { si } \alpha<\delta .
$$

Entonces, como el subespacio de $Y$

$$
Y_{\gamma}:=\varlimsup \operatorname{lin}\left\{u_{\delta}, x_{\delta}: \delta<\gamma\right\}
$$

es propio, el Lema de Riesz nos proporciona un elemento $x_{\gamma} \in S_{Y}$ con $d\left(x_{\gamma}, Y_{\gamma}\right)>s$ y, gracias al Teorema de Hahn-Banach, podemos elegir un funcional $x_{\gamma}^{*} \in S_{Y} \cap \cap Y_{\gamma}^{\circ}$ verificando $x_{\gamma}^{*}\left(x_{\gamma}\right)>s$. El Corolario 3.7 asegura la existencia de un subconjunto $\left\{\left(v_{\gamma}, v_{\gamma}^{*}\right): \gamma<\nu\right\} \subseteq S_{Y} \times S_{Y}$. que satisface

$$
\operatorname{Re} v_{\gamma}^{*}\left(v_{\gamma}\right)=v_{\gamma}^{*}\left(v_{\gamma}\right)>s
$$

y

$$
v_{\gamma}^{*}\left(v_{\delta}\right)=v_{\gamma}^{*}\left(u_{\delta}\right)=0, \text { si } \delta<\gamma .
$$

Sea $M$ una constante tal que $K-1>M>2 / s$. Como

$$
\left(u_{\gamma}^{*}+M v_{\gamma}^{*}\right)\left(u_{\gamma}\right)+\left(u_{\gamma}^{*}-M v_{\gamma}^{*}\right)\left(u_{\gamma}\right)=2
$$

si $\gamma<\nu$, podemos elegir $\varepsilon_{\gamma} \in\{-1,1\}$ tal que el funcional $z_{\gamma}^{*}=u_{\gamma}^{*}+\varepsilon_{\gamma} M v_{\gamma}^{*}$ cumpla $\left|z_{\gamma}^{*}\left(u_{\gamma}\right)\right| \geq 1$. Como no tenemos aún garantizada la existencia de una constante menor que uno que mayore a cada uno de los valores $\left|z_{\gamma}^{*}\left(u_{\delta}\right)\right|$ para $\gamma \neq \delta$ (hecho que perseguimos para que se tenga la propiedad $\beta$ ), intentaremos conseguir esa situación suprimiendo algunos de los funcionales. Para ello, fijamos un número real $r$, con $(M+1) / K<r<1$ y consideramos la familia $\mathcal{F}$ de subconjuntos de la forma

$$
B=\{1\} \cup\left\{\gamma<\nu:\left|z_{\delta}^{*}\left(u_{\gamma}\right)\right| \leq r, \text { si } \delta<\gamma, \delta \in B\right\} .
$$

Evidentemente $\mathcal{F}$ es no vacía y resulta inmediato comprobar que, ordenándola por inclusión, se convierte en un conjunto inductivo. Ei Lema 
de Zorn nos asegura la existencia de un elemento maximal $A$ de $\mathcal{F}$. Tomamos un número real

$$
D>\frac{2+M(1-s)}{1-r}
$$

y, para cada $\gamma \in A$, un escalar $\eta_{\gamma}$ tal que

$$
\left|\eta_{\gamma}\right|=1 \quad \text { y } \quad \varepsilon_{\gamma}\left|z_{\gamma}^{*}\left(u_{\gamma}\right)\right|=\eta_{\gamma} z_{\gamma}^{*}\left(u_{\gamma}\right) .
$$

Si definimos $z_{\gamma}:=v_{\gamma}+\eta_{\gamma} D u_{\gamma}$, para $\gamma \in A$, se verifica para $\delta, \gamma \in A$, con $\delta<\gamma$

$$
\begin{gathered}
\left|z_{\delta}^{*}\left(z_{\gamma}\right)\right|=\left|u_{\delta}^{*}\left(v_{\gamma}\right)+\varepsilon_{\delta} M v_{\delta}^{*}\left(v_{\gamma}\right)+\eta_{\gamma} D z_{\delta}^{*}\left(u_{\gamma}\right)\right| \leq \\
\leq 1+M+r D
\end{gathered}
$$

y

$$
\left|z_{\gamma}^{*}\left(z_{\delta}\right)\right|=\left|u_{\gamma}^{*}\left(v_{\delta}+\eta_{\delta} D u_{\delta}\right)\right| \leq 1+D,
$$

mientras que de (1) y (2) se tiene

$$
\begin{gathered}
\left|z_{\gamma}^{*}\left(z_{\gamma}\right)\right|=\left|u_{\gamma}^{*}\left(v_{\gamma}\right)+\varepsilon_{\gamma} M v_{\gamma}^{*}\left(v_{\gamma}\right)+\eta_{\gamma} D z_{\gamma}^{*}\left(u_{\gamma}\right)\right| \geq \\
\geq\left|\varepsilon_{\gamma} M v_{\gamma}^{*}\left(v_{\gamma}\right)+\eta_{\gamma} D z_{\gamma}^{*}\left(u_{\gamma}\right)\right|-\left|u_{\gamma}^{*}\left(v_{\gamma}\right)\right| \geq \\
\geq M s+D-1 .
\end{gathered}
$$

Los funcionales $y_{\gamma}^{*}:=\frac{1}{r} z_{\gamma}^{*}$, con $\gamma \in A$ serán los que verifiquen las condiciones de la propiedad $\beta$. Definimos

$$
\|y\|:=\sup \left\{\left|y_{\gamma}^{*}(y)\right|: \gamma \in A\right\} \quad(y \in Y),
$$

que es claramerite una seminorma en $Y$. Además, como

$$
\left\|y_{\gamma}^{*}\right\| \leq \frac{1+M}{r} \leq K
$$

entonces

$\|y\| \leq K\|y\|, \quad \forall y \in Y$ 
En particular, \|\|$\cdot \|$ es continua en $Y$ considerando la topología asociada a la norma de partida. De ahí que, para probar la desigualdad $\|y\| \leq\|y\|, \quad \forall y \in Y$, baste con demostrar que $\left\|u_{\gamma}\right\| \geq 1, \quad \forall \gamma<\nu$. Elijamos pues $\gamma<\nu$; si $\gamma \in A$, entonces

$$
\left\|u_{r}\right\| \geq \frac{1}{r} \geq 1
$$

mientras que si $\gamma \notin A$, debe existir $\delta \in A$ con $\delta<\gamma$ tal que

$$
\left|y_{\delta}^{*}\left(u_{\gamma}\right)\right| \geq 1
$$

con lo que también $\left\|u_{\gamma}\right\| \geq 1$. Así pues, $\|\cdot\|$ es una norma en $Y$ equivalente a la de partida. Tomando

y

$$
y_{\gamma}:=\frac{z_{\gamma}}{y_{\gamma}^{*}\left(z_{\gamma}\right)} \quad(\gamma \in A)
$$

$$
\lambda:=\frac{\max \{1+M+r D, 1+D\}}{M s+D-1}<1
$$

gracias a las condiciones (3), (4) y (5) se comprueba inmediatamente que $(Y,\|\cdot\| l i)$ verifica la propiedad $\beta$ para el conjunto $\left\{\left(y_{\gamma}, y_{\gamma}^{*}\right): \gamma \in A\right\}$ y la constante $\lambda$.

La única novedad de la demostración que hemos presentado con respecto a la de Partington consiste en hacer uso del Lema de Zorn para comprobar la existencia del conjunto de índices (que hemos llamado $A$ ), mientras que en la demostración original, se vuelve a usar el Principio de construcción transfinita.

Merece la pena mencionar que para espacios de Banach superreflexivos (admiten una norma equivalente que es uniformernente convexa) Partington mejora el resultado, consiguiendo probar el Teorema 3.9 para cada $K>1$. En vista de este hecho, consideramos la siguiente cuestión: 
3.10 Problema. iPuede asegurarse que si $(Y,\|\cdot\|)$ es un espacio de Banach y $K>1$, existe una norma $\|$.$\| en Y$ de forma que $(Y,\|\| \|$.$) verifica$ la propiedad $\beta$ y

$$
\|y\| \leq\|y\| \leq K\|y\|, \forall y \in Y ?
$$

Por supuesto, como sabemos -de acuerdo con el resultado de Lindenstrauss (Proposición 3.4)- que la propiedad $\beta$ es suficiente para la $B$, se tiene:

3.11 Corolario. Todo espacio de Banach es isomorfo a otro con la propiedad $B$.

Es fácil comprobar que si $Y$ es un espacio de Banach verificando la propiedad $\beta$ y de dimensión al menos dos, entonces no es estrictamente convexo. De hecho, no se conoce ni un sólo ejemplo de espacio de $\mathrm{Ba}$ nach de dimensión mayor que uno y estrictamente convexo que tenga la propiedad $B$. Surge así de manera natural el interrogante:

3.12 Problema. ¿Existe algún espacio de Banach estrictamente convexo y de dimensión mayor que uno que tenga la propiedad $B$ ?

Esta cuestión se ve acentuada aún más si cabe a la luz de esta otra:

3.13 Problema ([24, Question 6]). ¿Tiene $\mathbf{R}^{2}$ con la norma euclídea la propiedad $B$ ? 


\section{B. El Teorema de renormación de Schachermayer.}

En correspondencia con los a vtenidos de la sección anterior relativos a la propiedad $B$, daremos una condición de naturaleza geométrica, suficiente para la $A$ y un resultado que permite, bajo hipótesis muy suaves, renormar un espacio para que verifique la mencionada propiedad geométrica (y por tanto $A$ ).

Comenzamos introduciendo una nueva noción:

3.14 Definición ([27]). Sea $X$ un espacio de Banach. Se dice que un subconjunto $\left\{x_{i}: i \in I\right\}$ de $S_{X}$ es uniformemente fuertemente expuesto si existe un subconjunto $\left\{x_{i}^{*}: i \in I\right\}$ de $S_{X}$. verificando:

(a) $x_{i}^{*}\left(x_{i}\right)=1, \forall i \in I$.

(b) Para cada $\varepsilon>0$ existe $\delta>0$ de forma que si $i \in I$ y $x \in B_{X}$ verifica $\operatorname{Re} x_{i}^{*}(x)>1-\delta$, entonces se tiene que $\left\|x-x_{i}\right\|<\varepsilon$.

3.15 Ejemplo. Si $X$ es uniformemente convexo, entonces $S_{X}$ es un conjunto de puntos uniformemente fuertemente expuesto. Basta considerar, para cada $x \in S_{X}, x^{*} \in S_{X}$. tal que $x^{*}(x)=1$.

En primer lugar vamos a probar que si la bola unidad de un espacio de Banach $X$ se obtiene como el cierre de la envolvente absolutamente convexa de un conjunto uniformemente fuertemente expuesto, entonces $X$ tiene la propiedad $A$. Para tal fin, nos será de utilidad el siguiente lema técnico: 
3.16 Lema ([1, Lema 3.3]). Sea $\left\{x_{n}: n \in \mathbb{N}\right\}$ un subconjunto de la esfera unidad de un espacio de Banach $X$, que es uniformemente fuertemente expuesto por la familia de funcionales $\left\{x_{n}^{*}: n \in \mathbb{N}\right\}$ de $S_{X} \cdot y$ verifica

$$
\left|x_{m}^{*}\left(x_{n}\right)\right| \geq 1-\frac{1}{m}, \quad m<n .
$$

Entonces, la sucesión $\left\{x_{n}\right\}_{n \in \mathbb{N}}$ admite una parcial convergente (en norma).

Demostración. Para cada natural $n$, notamos

$$
\gamma_{n}=\inf _{k>n}\left|x_{n}^{*}\left(x_{k}\right)\right|
$$

por hipótesis la sucesión $\left\{\gamma_{n}\right\}_{n \in N}$ converge a uno.

Consideremos, ahora, para cada natural $k$, la sucesión $\alpha_{k}$ definida por

$$
\alpha_{k}(n)=x_{n}^{*}\left(x_{k}\right) \quad(n \in \mathbb{N})
$$

Por ser $\left\|x_{n}\right\|=\left\|x_{n}^{*}\right\|=1$ para todo natural $n,\left\{\alpha_{k}\right\}_{k \in N}$ es una sucesión de elementos de la bola unidad de $\ell_{\infty}$. Usando ahora que la topología $\omega^{*}$ de la bola unidad de $l_{\infty}$ es metrizable [23, Proposition 27.8], se consigue una sucesión parcial $\left\{\alpha_{\sigma(k)}\right\}_{k \in N}$ convergente en la topología $\omega^{*}$. Por tanto, para cada natural $n$, la sucesión $\left\{\alpha_{\sigma(k)}(n)\right\}_{k \in \mathbb{N}}=\left\{x_{n}^{*}\left(x_{\sigma(k)}\right)\right\}_{k \in \mathbb{N}}$ converge a un escalar $\lambda_{n}$. Para $n$ fijo y $k>n$, por ser $\sigma(k)>n$ se tiene

$$
1 \geq\left|x_{n}^{*}\left(x_{\sigma(k)}\right)\right| \geq \gamma_{n},
$$

de donde, haciendo $k \rightarrow \infty$ se obtiene

$$
1 \geq\left|\lambda_{n}\right| \geq \gamma_{n},
$$

y, por tanto, $\lim _{n \rightarrow \infty}\left|\lambda_{n}\right|=1$. 
Extraemos una parcial $\left\{\lambda_{\sigma(\tau(n))}\right\}_{n \in N}$ de la sucesión $\left\{\lambda_{\sigma(n)}\right\}_{n \in N}$ convergente a un escalar $\lambda$, que ha de tener módulo uno. Tomamos ahora

$$
y_{n}=x_{\sigma(\tau(n))}, y_{n}^{*}=x_{\sigma(\tau(n))}^{*}, \mu_{n}=\lambda_{\sigma(\tau(n))} \quad(n \in \mathbb{N}) .
$$

Por tenerse, para cada $n$ natural

$$
\lim _{k} x_{n}^{*}\left(x_{\sigma(k)}\right)=\lambda_{n},
$$

en particular se verifica

$$
\lim _{k} x_{\sigma(\tau(n))}^{*}\left(x_{\sigma(k)}\right)=\lambda_{\sigma(\tau(n))}=\mu_{n},
$$

y por tanto

$$
\lim _{k} x_{\sigma(\tau(n))}^{*}\left(x_{\sigma(\tau(k)))}\right)=\mu_{n}
$$

esto es,

$$
\lim _{k} y_{n}^{*}\left(y_{k}\right)=\mu_{n}, \quad \text { para cada natural } n,
$$

y evidentemente se tiene además $\lim _{n} \mu_{n}=\lambda$.

Probaremos ahora que $\left\{y_{n}\right\}_{n \in N}$ (sucesión parcial de $\left\{x_{n}\right\}_{n \in N}$ ) es convergente en norma.

Por ser $\left\{x_{n}: n \in \mathbb{N}\right\}$ un conjunto uniformemente fuertemente expuesto por los funcionales $\left\{x_{n}^{*}: n \in \mathbb{N}\right\}$, es claro que los conjuntos $\left\{y_{n}: n \in \mathbb{N}\right\}$ y $\left\{y_{n}^{*}: n \in \mathbb{N}\right\}$ también cumplen esta condición. Entonces, fijado $\varepsilon>0$, puede encontrarse un positivo $\delta$, verificando

$$
x \in B_{X}, \quad n \in \mathbb{N}, \quad \operatorname{Re} y_{n}^{*}(x)>1-\delta \Rightarrow\left\|x-y_{n}\right\|<\frac{\varepsilon}{2} .
$$

Por ser $\lim _{n} \mu_{n}=\lambda$, se tiene

$$
\exists n_{0} \in \mathbb{N}: n \geq n_{0} \Rightarrow\left|\mu_{n}-\lambda\right|<\frac{\delta}{2}
$$


Fijados ahora dos naturales $p$ y $q$ posteriores a $n_{0}$, usando que

$$
\left\{y_{p}^{*}\left(y_{k}\right)\right\}_{k \in N} \rightarrow \mu_{p} \text { y }\left\{y_{q}^{*}\left(y_{k}\right)\right\}_{k \in N} \rightarrow \mu_{q}
$$

tenemos

$$
\left|y_{p}^{*}\left(y_{k}\right)-\mu_{p}\right|<\frac{\delta}{2} \quad \text { y } \quad\left|y_{q}^{*}\left(y_{k}\right)-\mu_{q}\right|<\frac{\delta}{2}
$$

para $k$ suficientemente grande, $\mathrm{y}$, por tanto,

$$
\left|y_{p}^{*}\left(y_{k}\right)-\lambda\right|<\delta \text { y }\left|y_{q}^{*}\left(y_{k}\right)-\lambda\right|<\delta .
$$

Teniendo en cuenta que $\lambda$ tiene módulo uno, las expresiones anteriores se pueden escribir de la forma

$$
\left|y_{p}^{*}\left(\lambda^{-1} y_{k}\right)-1\right|<\delta \quad \text { y } \quad\left|y_{q}^{*}\left(\lambda^{-1} y_{k}\right)-1\right|<\delta
$$

luego

$$
\operatorname{Re} y_{p}^{*}\left(\lambda^{-1} y_{k}\right)>1-\delta \quad \text { y } \quad \operatorname{Re} y_{q}^{*}\left(\lambda^{-1} y_{k}\right)>1-\delta
$$

y usando ahora el hecho de que $\left\{y_{n}: n \in \mathbb{N}\right\}$ es una familia uniformemente fuertemente expuesta se tiene

$$
\left\|\left(\lambda^{-1} y_{k}\right)-y_{p}\right\|<\frac{\varepsilon}{2} \quad \text { y } \quad\left\|\left(\lambda^{-1} y_{k}\right)-y_{q}\right\|<\frac{\varepsilon}{2},
$$

de donde $\left\|y_{p}-y_{q}\right\|<\varepsilon$. Se ha probado que la sucesión $\left\{y_{n}\right\}_{n \in N}$ es de Cauchy, lo que concluye la demostración.

3.17 Proposición ([27, Proposition 1]). Sea $X$ un espacio de Banach para el cual existe un subconjunto de la esfera unidad $\left\{x_{i}: i \in I\right\}$ uniformemente fuertemente expuesto tal que el cierre de su envolvente absolutamente convexa coincide con la bola unidad de X. Entonces, $X$ tiene la propiedad $A$. 
Demostración. Al igual que en la demostración del Teorema 2.6, a partir de un operador construimos una sucesión de operadores que converge hacia otro, próximo al primero y que alcanza su norma. Sea pues $Y$ un espacio de Banach, $T$ un operador en $L(X, Y)$ con $\|T\|=1$ y $\varepsilon$ un número real y positivo con $0<\varepsilon<1$. Elegimos una sucesión decreciente $\left\{\varepsilon_{n}\right\}_{n \in N}$ de números reales positivos tal que

$$
\sum_{i=1}^{\infty} \varepsilon_{i}<\varepsilon / 2, \quad \sum_{i=n+1}^{\infty} \varepsilon_{i}<\varepsilon_{n}^{2} \quad \text { y } \quad \varepsilon_{n}<\frac{1}{10 n}
$$

para cada natural $n$. Tomamos $T_{1}=T \mathrm{y}$, supuesto definido $T_{n} \in L(X, Y)$, como $B_{X}=\overline{\operatorname{aco}}\left\{x_{i}: i \in 1\right\}$, elegimos un elemento $x_{n} \in\left\{x_{i}: i \in I\right\}$ tal que

$$
\left\|T_{n} x_{n}\right\|>\left\|T_{n}\right\|-\varepsilon_{n}^{2}
$$

y definimos

$$
T_{n+i}(x)=T_{n}(x)+\varepsilon_{n} x_{n}^{*}(x) T_{n} x_{n} \quad(x \in X),
$$

siendo $x_{n}^{*}$ el funcional asociado a $x_{n}$ que existe por ser $\left\{x_{i}: i \in I\right\}$ una familia uniformemente fuertemente expuesta. Es sencillo comprobar por inducción que

$$
\left\|T_{n+1}\right\| \leq 1+2 \sum_{i=1}^{n} \varepsilon_{i} \leq 2, \forall n \in \mathbb{N}
$$

de donde

$$
\left\|T_{n+1}-T_{m}\right\| \leq 2 \sum_{i=m}^{n} \varepsilon_{\imath}, \text { si } m \leq n .
$$

Por tanto, la sucesión $\left\{T_{n}\right\}_{n \in \mathbb{N}}$ converge hacia un operador $S \in L(X, Y)$ satisfaciendo

$$
\left\|S-T_{m}\right\| \leq 2 \sum_{i=m}^{\infty} \varepsilon_{i}
$$


en particular $\|S-T\|<\varepsilon$. Si $m<n$, entonces, usando (1) y (2),

$$
\begin{gathered}
\left\|T_{m+1} x_{n}\right\| \geq\left\|T_{n} x_{n}\right\|-\left\|T_{m+1}-T_{n}\right\| \geq \\
\geq\left\|T_{n}\right\|-\varepsilon_{n}^{2}-2 \sum_{i=m+1}^{n-1} \varepsilon_{i} \geq \\
\geq\left\|T_{m+1}\right\|-\varepsilon_{n}^{2}-4 \sum_{i=m+1}^{n-1} \varepsilon_{i} \geq \\
\geq\left\|T_{m+1}\right\|-5 \varepsilon_{m}^{2} \geq\left\|T_{m+1}\left(x_{m}\right)\right\|-5 \varepsilon_{m}^{2} \geq \\
\geq\left(1+\varepsilon_{m}\right)\left(\left\|T_{m}\right\|-\varepsilon_{m}^{2}\right)-5 \varepsilon_{m}^{2},
\end{gathered}
$$

de donde

$$
\begin{gathered}
\left\|T_{m}\right\|+\varepsilon_{m}\left\|T_{m}\right\|-7 \varepsilon_{m}^{2} \leq\left\|T_{m+1} x_{n}\right\| \leq \\
\leq\left\|T_{m}\right\|+\varepsilon_{m} \mid x_{m}^{*}\left(x_{n}\right)\left\|T_{m}\right\|
\end{gathered}
$$

y como de (2), $\left\|T_{m}\right\| \neq 0$, para cada $m$, entonces,

$$
\left|x_{m}^{*}\left(x_{n}\right)\right| \geq 1-7 \varepsilon_{m} \geq 1-\frac{1}{m}, \text { si } m<n .
$$

El Lema 3.16 nos asegura pues que la sucesión $\left\{x_{n}\right\}_{n \in N}$ admite una parcial convergente en la topología de la norma, hacia un vector $x \in S_{X}$. Como

$$
\begin{gathered}
\left\|S x_{n}\right\| \geq\left\|T_{n} x_{n}\right\|-\left\|S-T_{n}\right\| \geq \\
\geq\left\|T_{n}\right\|-\varepsilon_{n}^{2}-\left\|S-T_{n}\right\| \geq \\
\geq\|S\|-\varepsilon_{n}^{2}-2\left\|S-T_{n}\right\|,
\end{gathered}
$$

es claro que $\|S x\|=\|S\|$, y, por tanto, el operador $S$ alcanza la norma.

Si bien el resultado anterior da una condición suficiente para la propiedad $A$, es fácil dar un ejemplo de un espacio finito dimensional que no verifica la hipótesis: 
3.18 Ejemplo. Considérese el espacio $X=\mathbb{R}^{2}$ con la norma $\|\cdot\|$ cuya bola unidad es el conjunto $A=B_{2}+B_{\infty} \subseteq \mathbb{R}^{2}$, siendo $B_{2}$ y $B_{\infty}$ las bolas unidad para la norma euclídea y la norma del máximo, respectivamente.

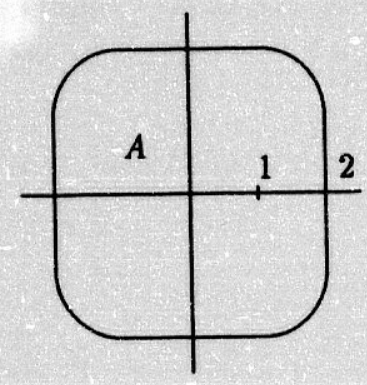

Inmediatamente se calcula la norma dual de $X:$ si $x^{*} \in X^{*}$,

$$
\begin{gathered}
\left\|x^{*}\right\|=\sup \left\{\left|x^{*}(u+v)\right|: u \in B_{2}, v \in B_{\infty}\right\}= \\
=\sup \left\{\left|x^{*}(u)\right|: u \in B_{2}\right\}+\sup \left\{\left|x^{*}(v)\right|: v \in B_{\infty}\right\}= \\
=\left\|x^{*}\right\|_{2}+\left\|x^{*}\right\|_{1} .
\end{gathered}
$$

Además, es claro que si $x \in S_{X}$ y $x^{*} \in S_{X}$. es un funcional tal que $x^{*}(x)=1$, poniendo $x=u+v$, con $u \in B_{2}$ y $v \in B_{\infty}$, ha de ser

$$
x^{*}(u)=\left\|x^{*}\right\|_{2} \text { y } \quad x^{*}(v)=\left\|x^{*}\right\|_{1} .
$$

Si $E \subseteq S_{X}$ es un conjunto uniformemente fuertemente expuesto, únicamente contiene puntos extremos de $B_{X}$, por tanto,

$$
E \subseteq\{(\cos \theta, \operatorname{sen} \theta)+( \pm 1, \pm 1): 0 \leq \theta \leq 2 \pi\} .
$$

Como además $E \cup-E \subseteq S_{X}$ es uniformemente fuertemente expuesto, puede suponerse $E=-E$. Si el elemento $(2,0) \in B_{X}$ estuviese en el 
cierre de la envolvente absolutamente convexa de $E$, entonces existe una sucesión $\left\{\theta_{n}\right\}_{n \in \mathbb{N}} \rightarrow 0$, con $0 \leq \theta_{n} \leq \pi / 2, \forall n \in \mathbb{N}$, tal que

$$
y_{n}:=(\cos \theta, \operatorname{sen} \theta)+(1,1) \in E, \quad \forall n \in \mathbb{N} .
$$

Si $\left\{y_{n}^{*}: n \in \mathbb{N}\right\}$ es la familia de funcionales que expone uniformemente a $\left\{y_{n}: n \in \mathbb{N}\right\}$, necesariamente debe ser

$$
y_{n}^{*}=\left(\frac{\cos \theta_{n}}{1+\operatorname{sen} \theta_{n}+\cos \theta_{n}}, \frac{\operatorname{sen} \theta_{n}}{1+\operatorname{sen} \theta_{n}+\cos \theta_{n}}\right) \quad(n \in \mathbb{N})
$$

y, por tanto,

$$
y_{n}^{*}(2,0)=\frac{2 \cos \theta_{n}}{1+\operatorname{sen} \theta_{n}+\cos \theta_{n}} \rightarrow 1
$$

mientras que

$$
\left\|(2,0)-y_{n}\right\|=\left\|\left(-1+\cos \theta_{n}, 1+\operatorname{sen} \theta_{n}\right)\right\| \rightarrow 1 .
$$

Así pues, $E$ no es un conjunto uniformemente fuertemente expuesto y $X$ no verifica la hipótesis de la Proposición 3.17.

A continuación presentamos una nueva propiedad de carácter geométrico, llamada propiedad $\alpha$, istroducida por W. Schachermayer, que es más restrictiva que la hipótesis de la Proposición 3.17 y, por tanto, también implica la $A$. Además, si un espacio de Banach $X$ tiene la propiedad $\alpha$, entonces $X^{*}$ tiene la $\beta$. Más adelante presentaremos un resultado de renormación para conseguir que se verifique $\alpha$.

3.19 Definición ([34, Definition 1.2.(a)]). Un espacio de Banach $X$ verifica la propiedad $\alpha$ si existe un conjunto $I$, un subconjunto $\left\{\left(x_{i}, x_{i}^{*}\right): i \in I\right\}$ de $S_{X} \times S_{X} \cdot$ y un número real $\lambda$, con $0 \leq \lambda<1$, tales que 
(a) $x_{i}^{*}\left(x_{i}\right)=1, \forall i \in I$.

(b) $\left|x_{i}^{*}\left(x_{j}\right)\right| \leq \lambda, \quad i \neq j, i, j \in I$.

(c) $B_{X}$ es el cierre de la envolvente absolutamente convexa del conjunto $\left\{x_{i}: i \in I\right\}$.

3.20 Ejemplo. El espacio de sucesiones $l_{1}$ tiene la propiedad $\alpha$ : basta considerar como conjunto de índices $\mathbb{N}$, constante $\lambda=0$ y el conjunto

$$
\left\{\left(e_{n}, e_{n}^{*}\right): n \in \mathbb{N}\right\} \subseteq S_{l_{1}} \times S_{l_{\infty}},
$$

siendo $\left\{e_{n}: n \in \mathbb{N}\right\}$ la base canónica de $l_{1}$ y $\left\{e_{n}^{*}: n \in \mathbb{N}\right\}$ el conjunto de funcionales asociados a dicha base.

3.21 Proposición ([34, Proposition 1.3]). Si un espacio de Banach satisface la propiedad $\alpha$, entonces también verifica la propiedad $A$.

Demostración. Al igual que W. Schachermayer, vamos a ofrecer dos demostraciones, una que usa la Proposición 3.17 y otra directa.

La primera demostración consiste en probar que el conjunto de puntos $\left\{x_{i}: i \in I\right\}$ está uniformemente fuertemente expuesto por el conjunto de funcionales $\left\{x_{i}^{*}: i \in I\right\}$, siendo ambos conjuntos los dados en la definición de la propiedad $\alpha$. Sea pues $\varepsilon>0, x \in B_{X} e i \in I$. Si $\eta>0$, de la definición de esta propiedad, se sigue la existencia de un natural $n$, escalares $\mu_{1}, \mu_{2}, \ldots, \mu_{n} \in \mathbb{K}$, con $\sum_{j=1}^{n}\left|\mu_{j}\right| \leq 1$ y $n$ vectores distintos $x_{1}, x_{2}, \ldots, x_{n} \in\left\{x_{i}: i \in I\right\}$ verificando

$$
x_{0}=\sum_{j=1}^{n} \mu_{j} x_{j} \quad \text { y } \quad\left\|x-x_{0}\right\|<\eta
$$


Podemos suponer sin pérdidad de generalidad que $x_{1}=x_{i}$. Entonces,

$$
\begin{gathered}
\left\|x_{0}-x_{i}\right\|=\left\|\left(\mu_{1}-1\right) x_{i}+\sum_{j=2}^{n} \mu_{j} x_{j}\right\| \leq \\
\leq\left|1-\mu_{1}\right|+1-\left|\mu_{1}\right| \leq 2\left|1-\mu_{1}\right| .
\end{gathered}
$$

Queremos probar que existe un positivo $\delta>0$ (independiente de $i$ ) que cumple

$$
\left\|x-x_{i}\right\|<\varepsilon, \text { si } \operatorname{Re} x_{i}^{*}(x)>1-\delta .
$$

Supongamos pues que $\delta>0$ verifica Re $x_{i}^{*}(x)>1-\delta$; entonces,

$$
\operatorname{Re} x_{i}^{*}\left(x_{0}\right)=\operatorname{Re} x_{i}^{*}(x)-\operatorname{Re} x_{i}^{*}\left(x-x_{0}\right)>1-\delta-\eta
$$

y por tanto,

$$
\begin{gathered}
1-\delta-\eta<\operatorname{Re} x_{i}^{*}\left(x_{0}\right)=\operatorname{Re} x_{i}^{*}\left(\mu_{1} x_{i}+\sum_{j=2}^{n} \mu_{j} x_{i}^{*}\left(x_{j}\right)\right) \leq \\
\leq \operatorname{Re} x_{i}^{*}\left(\mu_{1} x_{i}\right)+\sum_{j=2}^{n}\left|\mu_{j}\right| \lambda \leq \operatorname{Re}\left(\mu_{1}\right)+\left(1-\left|\mu_{1}\right|\right) \lambda \leq \\
\leq \operatorname{Re}\left(\mu_{1}\right)+\left(1-\operatorname{Re}\left(\mu_{1}\right)\right) \lambda
\end{gathered}
$$

siendo $\lambda$ la constante que proporciona la definición de la propiedad $\alpha$. Así pues, la desigualdad anterior nos da

$$
1-\operatorname{Re}\left(\mu_{1}\right) \leq \frac{\delta+\eta}{1-\lambda}
$$

de donde

$$
\left|1-\mu_{1}\right|^{2}=1+\left|\mu_{1}\right|^{2}-2 \operatorname{Re}\left(\mu_{1}\right) \leq 2-2\left(1-\frac{\delta+\eta}{1-\lambda}\right) .
$$


De (1), (2) y (3), podemos obtener una estimación de la distancia de $x$ a $x_{i}$ :

$$
\begin{gathered}
\left\|x-x_{i}\right\| \leq\left\|x-x_{0}\right\|+\left\|x_{0}-x_{i}\right\| \leq \\
\leq \eta+2\left|1-\mu_{1}\right| \leq \eta+2\left(2 \frac{\delta+\eta}{1-\lambda}\right)^{1 / 2} .
\end{gathered}
$$

Sin más que toinar $\eta$ y $\delta$ tales que

$$
\eta+2\left(2 \frac{\delta+\eta}{1-\lambda}\right)^{1 / 2}<\varepsilon
$$

se tiene

$$
\left\|x-x_{i}\right\|<\varepsilon .
$$

Daremos ahora la demostración directa. Sea $X$ un espacio de Banach con la propiedad $\alpha, Y$ un espacio de Banach cualquiera, $T \in L(X, Y)$ con $\|T\|=1$ y $\varepsilon>0$. Puesto que de la condición (c) en la Definición 3.19 se tiene

$$
\|T\|=\sup \left\{\left\|T x_{i}\right\|: i \in I\right\},
$$

si elegimos un número real $\eta$ verificando $0<\eta<1$, existe $i \in I$ tal que $\left\|T x_{i}\right\|>1-\eta$. Definimos entonces

$$
S x:=T x+\varepsilon x_{i}^{*}(x) T x_{i} \quad(x \in X) .
$$

Es claro que $S \in L(X, Y),\|S-T\|<\varepsilon$ y además,

$$
\left\|S x_{i}\right\|=(1+\varepsilon)\left\|T x_{i}\right\|>(1+\varepsilon)(1-\eta)
$$

mientras que si $j \neq i$, entonces

$$
\left\|S x_{j}\right\| \leq 1+\varepsilon \lambda .
$$

Así pues, tomando $\eta$ de forma que $(1+\varepsilon)(1-\eta) \geq 1+\varepsilon \lambda, S$ alcanza su norma en $x_{i}$. 
La primera de las dos demostraciones pone de manifiesto que la propiedad $\alpha$ no es más que una condición suficiente para que la bola unidad del espacio sea el cierre de la envolvente absolutamente convexa de un conjunto uniformemente fuertemente expuesto. Obsérvese que en la segunda se consigue, de hecho, a partir de un operador $T \in L(X, Y)$ otro operador $S \in L(X, Y)$ que alcanza su norma, está próximo a $T$ y $S-T$ es un operador de rango uno.

Estudiemos ahora la relación existente entre las propiedades $\alpha$ y $\beta$.

3.22 Definición ([34]). Se dice que un espacio de Banach $Y$ tiene la propiedad $\beta$ inducida por un subespacio $Z$ de $Y^{*}$ si $Y$ verifica la propiedad $\beta$ para un conjunto $\left\{\left(y_{i}, y_{i}^{*}\right) \in S_{Y} \times S_{Y} \bullet: i \in I\right\}$ tal que el subconjunto $\left\{y_{i}^{*}: i \in I\right\}$ está contenido en $Z$.

3.23 Proposición ([34, Proposition 1.4]). Un espacio de Banach $X$ tiene la propiedad $\alpha$ si, $y$ sólo si, $X^{*}$ verifica la propiedad $\beta$ inducida por $X$.

Demostración. Si $X$ tiene la propiedad $\alpha$ para la familia

$$
\left\{\left(x_{i}, x_{i}^{*}\right): i \in I\right\}
$$

y $0 \leq \lambda<1$ (Definición 3.19), entonces, por ser

$$
B_{X}=\overline{\operatorname{aco}}\left\{x_{i}: i \in I\right\} \text {, }
$$

se tiene

$$
\left\|x^{*}\right\|=\sup \left\{\left|x^{*}\left(x_{i}\right)\right|: i \in I\right\}, \forall x^{*} \in X^{*},
$$

con lo que $X^{*}$ satisface la propiedad $\beta$ inducida por $X$ para el conjunto $\left\{\left(x_{i}^{*}, x_{i}\right): i \in I\right\}$ y la misma constante. 
Recíprocamente, si $X^{*}$ verifica la propiedad $\beta$ inducida por $X$, existe un subconjunto $\left\{\left(x_{i}^{*}, x_{i}\right): i \in I\right\} \subseteq X^{*} \times X$ satisfaciendo las condiciones de la Definición 3.2. Sabemos entonces que

$$
\left\|x^{*}\right\|=\sup \left\{\left|x^{*}\left(x_{i}\right)\right|: i \in I\right\}, \forall x^{*} \in X^{*}
$$

por lo que

$$
B_{X} \cdot=\left(\left\{x_{i}: i \in I\right\}\right)^{\circ}
$$

luego, en virtud del Teorema del bipolar, se tiene

$$
B_{X}=\overline{\operatorname{aco}}\left\{x_{i}: i \in I\right\} \text {, }
$$

$y$, por tanto, $X$ tiene la propiedad $\alpha$.

3.24 Corolario. Un espacio de Banach reflexivo $X$ tiene la propiedad $\alpha$ (resp. $\beta$ ) si, y sólo si, $X^{*}$ tiene la $\beta$ (resp. $\alpha$ ).

Consecuentemente, el espacio euclídeo de dimensión dos no tiene la propiedad $\alpha \mathrm{y}$, sin embargo, como es uniformemente convexo, su bola unidad se obtiene como el cierre de la envolvente absolutamente convexa de un subconjunto uniformemente fuertemente expuesto. Además, con respecto a la otra condición suficiente para la propiedad $A$ que conocemos, la reflexividad, la hipótesis usada en la Proposición 3.17 nc guarda relación alguna: como hemos probado, hay espacios reflexivos (finito dimensionales) sin esa propiedad; por otro lado, $l_{1}$ satisface la propiedad $\alpha$ y no es reflexivo.

Si bien el problema de renormar un espacio de Banach con la propiedad $B$ (de hecho la $\beta$ ) fue resuelto afirmativamente por Partington, para el problema análogo con la propiedad $A$ únicamente disponemos de un resultado parcial, debido a $W$. Schachermayer. 
El Teorema de Schachermayer resuelve afirmativamente enormación con la propiedad $\alpha$ de una amplia clase de espacios de Banach. Para precisar cuáles son dichos espacios se necesita introducir el siguiente concepto.

3.25 Definición ([9, Definition VI.1.1]). Sea $X$ un espacio de Banarh infinito dimensional y $\mu$ un ordinal tal que $|\mu|=\operatorname{dens}(X)$. Una resolución proyectiva de la identidad en $X$ es una familia de proyecciones en $X\left\{P_{\alpha}: \omega_{0} \leq \alpha \leq \mu\right\}$ de forma que

(i) $\left\|P_{\alpha}\right\|=1, \quad \forall \omega_{0} \leq \alpha \leq \mu$.

(ii) $P_{\alpha} P_{\beta}=P_{\beta} P_{\alpha}=P_{\alpha}$, si $\omega_{0} \leq \alpha \leq \beta \leq \mu$.

(iii) $\operatorname{dens}\left(P_{\alpha}(X)\right) \leq|\alpha|$.

(iv) $U\left\{P_{\beta+1}(X): \beta<\alpha\right\}$ es denso (en norma) en $P_{\alpha}(X)$.

(v) $P_{\mu}=I_{X}$.

El resultado sobre renormación que vamos a probar asegura que todo espacio de Banach con una resolución proyectiva de la identidad, es isomorfo a otro espacio de Banach que verifica la propiedad $\alpha$. Presentemos algunos ejemplos de este tipo de espacios.

3.26 Definición. Se dice que un espacio de Banach $X$ es débilmente compactamente generado (W.C.G.) si existe un subconjunto $K$ de $X$ débilmente compacto tal que

$$
X=\longdiv { \operatorname { l i n } ( K ) }
$$


Es claro que si $X$ es reflexivo o separable, entonces es W.C.G.

En el texto [9] se prueba que todo espacio de Banach W.C.G. admite una resolución proyectiva de la identidad.

Si $X$ es un espacio de Banach con una resolución proyectiva de la identidad, necesitamos conseguir un conjunto de vectores y funcionales con ciertas propiedades para renormar $\operatorname{con} \alpha$.

Comenzamos probando la existencia de sucesiones básicas en cualquier espacio de Banach.

3.27 Lema ([10, Lemma V.2]). Sea $X$ un espacio de Banach infinito dimensional, $F$ un subespacio suyo finito dimensional $y \varepsilon>0$. Entonces, existe un vector $x \in X \backslash F$ tal que $\|x\|=1 y$

$$
\|y\| \leq(1+\varepsilon)\|y+\lambda x\|, \quad \forall y \in F, \lambda \in \mathbb{K}
$$

Demostración. Tomemos $\varepsilon \leq 1$. Sea $\left\{y_{1}, \ldots, y_{n}\right\} \subseteq S_{F}$ tal que

$$
\min \left\{\left\|y-y_{i}\right\|: i=1,2, \ldots, n\right\} \leq \frac{\varepsilon}{2}, \forall y \in S_{F}
$$

e $\left\{y_{1}^{*}, \ldots, y_{n}^{*}\right\} \subseteq S_{F^{*}}$ de modo que $y_{i}^{*}\left(y_{i}\right)=1, i=1,2, \ldots, n$. Basta tomar $x \in \cap_{i=1}^{n} \operatorname{ker} y_{i}^{*}$ con $\|x\|=1$. Sea $\lambda \in \mathbb{K}$ e $y \in F$, que podemos suponer de norma uno. Entonces fijamos $i$ tal que $\left\|y-y_{i}\right\| \leq \frac{\varepsilon}{2}$, con lo que

$$
\begin{gathered}
\|y+\lambda x\| \geq\left\|y_{i}+\lambda x\right\|-\left\|y-y_{i}\right\| \geq \\
\geq y_{i}^{*}\left(y_{i}+\lambda x\right)-\varepsilon / 2=1-\varepsilon / 2 \geq \frac{1}{1+\varepsilon}
\end{gathered}
$$

3.28 Corolario ([10, Corollary V.3]). Dado $K>1$, todo espacio de Binach infinito dimensional posee una sucesión básica con constante básica menor o igual que $K$. 
Demostración. Sea $X$ un espacio de Banach y $0<\varepsilon<K-1$. Elijamos una sucesión de números reales positivos $\left\{\varepsilon_{n}\right\}_{n \in N}$ tal que

$$
\Pi_{n=1}^{\infty}\left(1+\varepsilon_{n}\right) \leq 1+\varepsilon
$$

Fijamos un vector $x_{1}$ de $S_{X}$ y llamamos $F_{1}$ al subespacio de $X$ generado por $x_{1}$. El lema previo nos proporciona un vector $x_{2} \in S_{X}$ verificando

$$
\|y\| \leq\left(1+\varepsilon_{1}\right)\left\|y+\lambda x_{2}\right\|_{i}, \forall y \in F_{1}, \forall \lambda \in \mathbb{K} .
$$

Por recurrencia obtenemos una sucesión $\left\{x_{n}\right\}_{n \in N}$ de vectores de $S_{X}$ de forma que

$$
\|y\| \leq\left(1+\varepsilon_{n}\right)\left\|y+\lambda x_{n+1}\right\|, \forall y \in F_{n}=\operatorname{lin}\left\{x_{1}, \ldots x_{n}\right\}, \forall \lambda \in \mathbb{K} .
$$

Entonces es obvio que $\left\{r_{n}\right\}_{n \in \mathbb{N}}$ es una sucesión básica de $X$ con constante básica menor o igual que $\Pi_{n=1}^{\infty}\left(1+\varepsilon_{n}\right) \leq 1+\varepsilon \leq K$.

Usaremos la noción que ahora introducimos en algunos resultados que siguen.

3.29 Definición. Dos sucesiones $\left\{x_{n}\right\}_{n \in \mathbb{N}}$ e $\left\{y_{n}\right\}_{n \in \mathbb{N}}$ en sendos espacios de Banach $X$ e $Y$ se dicen equivalentes cuando para toda sucesión de escalares $\left\{\lambda_{n}\right\}_{n \in \mathbb{N}}$, la serie $\sum_{n \in \mathbb{N}} \lambda_{n} x_{n}$ es convergente si, y sólo si, lo es la serie $\sum_{n \in \mathbb{N}} \lambda_{n} y_{n}$.

3.30 Ejemplo. Consideremos en $l_{1}$ su base usual $\left\{e_{n}\right\}_{n \in \mathrm{N}}$ y la sucesión $\left\{y_{n}\right\}_{n \in \mathrm{N}}$ definida como

$$
\left\{\begin{array}{l}
y_{1}=e_{1} \\
y_{n+1}=e_{n+1}-e_{n}, \quad \forall n \in \mathbb{N}
\end{array}\right.
$$

Estas sucesiones no son equivalentes (tómese en la definición $\lambda_{n}=1 / n$, para todo $n$ natural). 
El siguiente resultado nos permite encontrar en espacios de Banach isomorfos a $l_{1}$ sucesiones básicas equivalentes a la base canónica de $l_{1}$, de manera que es posible controlar la norma del isomorfismo:

3.31 Lema ([28, Proposition 2.e.3]). Sea |||.|| una norma en $l_{1}$ equivalente a la usual. Entonces, para cada $\varepsilon>0$ existe una sucesión básica $\left\{y_{n}\right\}_{n \in \mathbb{N}}$ verificando

$$
\left\|y_{n}\right\|=1, \forall n \in \mathbb{N}
$$

$y$

$$
(1+\varepsilon)^{-2}\|x\|_{1} \leq\left\|\sum_{n=1}^{\infty} x(n) y_{n}\right\| \leq\|x\|_{1}, \quad \forall x \in l_{1} .
$$

Además existe una aplicación $\sigma: \mathbb{N} \longrightarrow \mathbb{N}$ estrictamente creciente y una sucesión de escalares $\left\{\alpha_{n}\right\}_{n \in \mathbb{N}}$ de modo que

$$
y_{n}=\sum_{j=\sigma(n)+1}^{\sigma(n+1)} \alpha_{j} e_{j}, \forall n \in \mathbb{N},
$$

donde $\left\{e_{n}\right\}_{n \in N}$ es la base usual de $l_{1}$.

Demostración. Por hipótesis, existen $\alpha, \beta \in \mathbb{R}^{+}$verificando

$$
\alpha\|x\| \leq\|x\|_{1} \leq \beta\|x\| \quad\left(x \in l_{1}\right) .
$$

Notaremos por $\left\{P_{n}\right\}_{n \in \mathbb{N}}$ a la sucesión de proyecciones asociada a la base canónica de $l_{1}$. Dado $n \in \mathbb{N}$ definimos

$$
\lambda_{n}=\sup \left\{\|x\|_{1}:\|x\|=1, P_{n} x=0\right\} .
$$

Claramente la sucesión $\left\{\lambda_{n}\right\}_{n \in \mathbb{N}}$ es decreciente y converge hacia un número real $\lambda \in[\alpha, \beta]$. Dado $\varepsilon>0$, existe entonces un natural $\sigma(1)$ tal que

$$
\lambda_{\sigma(1)}<\lambda(1+\varepsilon) \text {. }
$$


Construimos la nueva base por recurrencia. Por definición de $\lambda_{\sigma(1)}$, existe $x_{1} \in l_{1}$ tal que $\left\|x_{1}\right\|=1,\left\|x_{1}\right\|_{1}>\lambda(1+\varepsilon)^{-1}$ y $P_{\sigma(1)} x_{1}=0$. Sea $\eta \in \mathbb{R}^{+}$ satisfaciendo la desigualdad

$$
(1+\eta) \lambda(1+\varepsilon)^{-1}<\left\|x_{1}\right\|_{1} .
$$

Entonces, existe $\sigma(2)>\sigma(1)$ tal que $z_{1}=\sum_{j=\sigma(1)+1}^{\sigma(2)} x_{1}(j) e_{j}$ verifica

$$
(1+\eta) \lambda(1+\varepsilon)^{-1}<\left\|z_{1}\right\|_{1} \leq\left\|x_{1}\right\|_{1} \text { y }\left\|z_{1}-x_{1}\right\|<\eta .
$$

Definimos $y_{1}=z_{1} /\left\|z_{1}\right\|$. Entonces, $P_{\sigma(1)} y_{1}=0,\left\|y_{1}\right\|=1$ y

$$
\left\|y_{1}\right\|_{1}=\frac{\left\|z_{1}\right\|_{1}}{\left\|z_{1}\right\|}>\frac{(1+\eta) \lambda(1+\varepsilon)^{-1}}{1+\eta}=\frac{\lambda}{1+\varepsilon}
$$

Supuesto definido $\sigma(n)$, como $\lambda_{\sigma(n)}>\frac{\lambda}{1+\varepsilon}$, existe $x_{n+1} \in l_{1}$ verificando

$$
\left\|x_{n+1}\right\|=1,\left\|x_{n+1}\right\|_{1}>\frac{\lambda}{1+\varepsilon} \text { y } P_{\sigma(n)} x_{n+1}=0 .
$$

Repitiendo el mismo argumento del caso $n=1$, encontramos un vector $y_{n+1} \in \operatorname{lin}\left\{e_{\sigma(n)+1}, \ldots, e_{\sigma(n+1)}\right\}$, para cierto natural $\sigma(n+1)>\sigma(n)$ tal que

$$
\left\|y_{n+1}\right\|=1,\left\|y_{n+1}\right\|_{1}>\frac{\lambda}{1+\varepsilon} \text { y } P_{\sigma(n+1)} y_{n+1}=0 .
$$

Por tanto, $\left\{y_{n}\right\}_{n \in N}$ es una sucesión básica obtenida de la base usual de $l_{1}$ como indica (1), que verifica

$$
\left\|y_{n}\right\|=1, \quad P_{\sigma(n)} y_{n}=0 \text { y }\left\|y_{n}\right\|_{1}>\frac{\lambda}{1+\varepsilon}, \forall n \in \mathbb{N}
$$

Entonces, si $x \in l_{1}$, y puesto que $P_{\sigma(1)}\left(\sum_{n=1}^{\infty} x(n) y_{n}\right)=0$, tenemos

$$
\left\|\sum_{n=1}^{\infty} x(n) y_{n}\right\| \geq \lambda_{\sigma(1)}^{-1}\left\|\sum_{n=1}^{\infty} x(n) y_{n}\right\|_{1}=
$$




$$
\begin{gathered}
=\lambda_{\sigma(1)}^{-1} \sum_{n=1}^{\infty} \mid x(n)\left\|y_{n}\right\|_{1} \geq \\
\geq \lambda_{\sigma(1)}^{-1} \lambda(1+\varepsilon)^{-1} \sum_{n=1}^{\infty}|x(n)| \geq(1+\varepsilon)^{-2} \sum_{n=1}^{\infty}|x(n)|,
\end{gathered}
$$

y obviamente$$
\left\|\sum_{n=1}^{\infty} x(n) y_{n}\right\| \leq \sum_{n=1}^{\infty}|x(n)|\left\|y_{n}\right\|\left|=\sum_{n=1}^{\infty}\right| x(n) \mid .
$$

Los resultados anteriores nos permiten construir en un espacio de Banach una familia de vectores y funcionales que, si bien no es exactamente la buscada, sí resuelve la determinación de ésta.

3.32 Lema. Sea $X$ un espacio de Banach infinito dimensional. Dado $\varepsilon>0$, existe un conjunto $\left\{\left(y_{n}, y_{n}^{*}\right) \in X \times X^{*}: n \in \mathbb{N}\right\}$ tal que

(a) $\left\|y_{n}\right\|=1,\left\|y_{n}^{*}\right\| \leq 2+\varepsilon, \quad \forall n \in \mathbb{N}$,

(b) $y_{n}^{*}\left(y_{n}\right)=1, \quad y_{n}^{*}\left(y_{m}\right)=0$, si $n \neq m$

(c) la sucesión $\left\{y_{n}\right\}_{n \in N}$ no es equivalente a la base usual de $l_{1}$.

Demostración. Dado $0<\eta<1$, en virtud del Corolario 3.28 existe una sucesión básica en $X,\left\{x_{n}\right\}_{n \in N}$, con funcionales biortogonales $\left\{x_{n}^{*}\right\}_{n \in \mathbf{N}}$, de modo que

$$
\left\|x_{n}\right\|=1 \text { y }\left\|x_{n}^{*}\right\| \leq 2+\eta, \quad \forall n \in \mathbb{N} .
$$

En caso de que $\left\{x_{n}\right\}_{n \in \mathrm{N}}$ no sea equivalente a la base canónica de $l_{1}$, el conjunto $\left\{\left(x_{n}, x_{n}^{*}\right): n \in \mathbb{N}\right\}$ ya verifica (a), (b) y (c) sin más que tomar 
$\eta=\varepsilon$. Supongamos entonces que $\left\{x_{n}\right\}_{n \in N}$ es equivalente a la base natural de $l_{1} ;$ por tanto, el operador

$$
T: l_{1} \longrightarrow \varlimsup \operatorname{in}\left\{x_{n}: n \in \mathbb{N}\right\}
$$

definido por

$$
T \omega=\sum_{n=1}^{\infty} \omega(n) x_{n} \quad\left(\omega \in l_{1}\right)
$$

es un isomorfismo. Nótese, en primer lugar, que a partir de un conjunto que verifique (a), (b) y (c) en un subespacio de $X$, inmediatamente se obtiene la tesis buscada, sin más que extender los funcionales usando el Teorema de Hahn-Banach. Podemos suponer, por tanto,

$$
X=\varlimsup i n\left\{x_{n}: n \in \mathbb{N}\right\} .
$$

Como además $X$ y $\left(l_{1},\|\cdot\| \|\right)$ son isométricos, siendo

$$
\|\omega\|=\|T \omega\| \quad\left(\omega \in l_{1}\right),
$$

basta probar la tesis para $X=\left(l_{1},\|\cdot\| \cdot \|\right)$. El Ejemplo 3.30 nos proporciona una sucesión de $l_{1}$ que verifica $(\mathrm{c})$. Como pretendemos que también se dé (a), tendremos que proceder con algo más de cuidado. Primeramente aplicamos el Lema 3.31 obteniendo una sucesión básica $\left\{z_{n}\right\}_{n \in \mathbb{N}}$ en $l_{1}$, equivalente a la usual, tal que $\left\|z_{n}\right\|=1$, para cada $n$ y

$$
(1-\eta) \sum_{n=1}^{\infty}|\omega(n)| \leq \mathbb{\|} \sum_{n=1}^{\infty} \omega(n) z_{n}\left|\| \leq \sum_{n=1}^{\infty}\right| \omega(n) \mid, \quad \forall \omega \in l_{1} .
$$

Hacemos ahora el mismo cambio que en el Ejemplo 3.30, es decir, tomamos

$$
\left\{\begin{array}{l}
\omega_{1}=z_{1} \\
\omega_{n+1}=z_{n+1}-z_{n}, \quad \forall n \in \mathbb{N} .
\end{array}\right.
$$

Es claro que la sucesión $\left\{\omega_{n}\right\}_{n \in \mathbb{N}}$ no es equivalente a la base canónica de $l_{1} \mathrm{y}$, además, teniendo en cuenta $(1)$, sabemos que

$$
(1-\eta) \leq\left\|\omega_{n}\right\| \leq 2, \forall n \in \mathbb{N} .
$$


Si para cada $n$ definimos

$$
\omega_{n}^{*}(y)=\sum_{m=n}^{\infty} z_{m}^{*}(y) \quad\left(y \in l_{1}\right),
$$

entonces se comprueba inmediatamente que

$$
\omega_{n}^{*}\left(\omega_{n}\right)=1 \quad(n \in \mathbb{N})
$$

y

$$
\omega_{n}^{*}\left(\omega_{m}\right)=0 \quad(n \neq m)
$$

Probemos ahora que, considerando el subespacio de $l_{1}$ dado por

$$
\varlimsup i n\left\{z_{n}: n \in \mathbb{N}\right\},
$$

se verifica que $\left\|\omega_{n}^{*}\right\| \leq \frac{1}{1-\eta}$, para todo $n$ natural. En efecto, si $y \in \mathbb{\operatorname { l i n }}\left\{z_{n}: n \in \mathbb{N}\right\}$, entonces

$$
\begin{gathered}
\left|\omega_{n}^{*}(y)\right|=\left|\sum_{m=n}^{\infty} z_{m}^{*}(y)\right| \leq \sum_{m=1}^{\infty}\left|z_{m}^{*}(y)\right| \leq \text { por }(1) \\
\leq \frac{1}{1-\eta}\left\|\sum_{m=1}^{\infty} z_{m}^{*}(y) z_{m}\right\| \mid=\frac{1}{1-\eta}\|y\| .
\end{gathered}
$$

Como la sucesión $\left\{\omega_{n}\right\}_{n \in N}$ no es equivalente a la base usual de $l_{1}$, usando (2) se tiene que tampoco lo es la sucesión $\left\{\frac{\omega_{n}}{\left\|\omega_{n}\right\|}\right\}$. Basta tomar entonces

$$
y_{n}=\frac{\omega_{n}}{\left\|\omega_{n}\right\|}, \quad y_{n}^{*}=\left\|\omega_{n}\right\| \omega_{n}^{*}, \quad \forall n \in \mathbb{N},
$$

para obtener un conjunto verificando (a), (b) y (c), sin más que elegir $\eta$ tal que $2 /(1-\eta)<2+\varepsilon$.

Aplicando el lema anterior convenientemente obtenemos: 
3.33 Lema fundamental ([15, Lemma]). Sea $X$ un espacio de Banach, con carácter de densidad dens $(X)=|I|$, que admite una resolución proyectiva de la identidad. Entonces, para cada $\varepsilon>0$ existe un conjunto $\left\{\left(y_{i, n}, y_{i, n}^{*}\right) \in X \times X^{*}: i \in I, n \in \mathbb{N}\right\}$ verificando

(a) $\left\|y_{i, n}\right\| \leq 1, \quad\left\|y_{i, n}^{*}\right\| \leq 4+\varepsilon, \quad \forall i \in I, n \in \mathbb{N}$.

(b) $y_{i, n}^{*}\left(y_{i, n}\right)=1, \quad y_{i, n}^{*}\left(y_{j, m}\right)=0$, si $(i, n) \neq(j, m)$.

(c) Para todo $i \in I$, la sucesión $\left\{y_{i, n}\right\}_{n \in \mathbb{N}}$ no es equivalente a la base canónica de $l_{1}$.

Demostración. Si $X$ posee una resolución proyectiva de la identidad, podemos encontrar una familia de proyecciones $\left\{P_{i}: i \in I\right\}$ que verifica

$$
\begin{gathered}
\left\|P_{i}\right\|=1, \quad \forall i \in I, \\
P_{i} P_{j}=P_{j} P_{i}=P_{j}, \quad \text { si } j<i
\end{gathered}
$$

y

$$
\operatorname{dim}\left(P_{i+1}-P_{i}\right)(X)=\infty .
$$

Sea $\varepsilon>0$. Si para cada $i \in I$ notamos

$$
X_{i}=\left(P_{i+1}-P_{i}\right)(X),
$$

entonces $X_{i}$ es un espacio de Banach infinito dimensional y podemos aplicar el Lema 3.32, obteniendo una sucesión

$$
\left\{\left(y_{i, n}, \hat{y}_{i, n}^{*}\right) \in X_{i} \times X_{i}^{*}: n \in \mathbb{N}\right\}
$$

de modo que

$$
\begin{aligned}
& \left\|y_{i, n}\right\|=1, \quad\left\|\hat{y}_{i, n}^{*}\right\| \leq 2+\varepsilon / 2, \quad \forall n \in \mathbb{N} \\
& \hat{y}_{i, n}^{*}\left(y_{i, n}\right)=1, \quad \hat{y}_{i, n}^{*}\left(y_{i, m}\right)=0, \quad \text { si } n \neq m
\end{aligned}
$$


y la sucesión $\left\{y_{i, n}\right\}_{n \in \mathbb{N}}$ no equivale a la base usual de $l_{1}$. Dados $i \in I$ y $n \in \mathbb{N}$, definimos

$$
y_{i, n}^{*}=\hat{y}_{i, n}^{*} \circ\left(P_{i+1}-P_{i}\right) \in X^{*} .
$$

Entonces, se comprueba inmediatamente que el sistema

$$
\left\{\left(y_{i, n}, y_{i, n}^{*}\right) \in X \times X^{*}: i \in I, n \in \mathbb{N}\right\}
$$

verifica la tesis del enunciado.

3.34 Teorema ([34, Theorem 4.4]). Si $(X,\|\|$.$) es un espacio de Banach$ con una resolución proyectiva de la identidad, existe una norma $\|\mid\| \cdot \|$ en $X$ equivalente a la de partida $y$ de forma que $(X,\|\| \cdot \|)$ tiene la propiedad $\alpha$. De hecho, para todo $0<\lambda<1$ se puede conseguir que la constante de la definición sea $\lambda$.

Demostración. Sea $0<\lambda\langle 1, \varepsilon>0$ e $I$ tal que $|I|=\operatorname{dens}(X)$. El Lema fundamental nos proporciona, para cada número real positivo $\varepsilon$, un conjunto

$$
\left\{\left(y_{i, n}, y_{i, n}^{*}\right): i \in I, n \in \mathbb{N}\right\}
$$

verificando las condiciones (a), (b) y (c) del Lema fundamental. Como para cada $i$, la sucesión $\left\{y_{i, n}\right\}_{n \in N}$ es acotada y no equivalente a la base usual de $l_{1}$, existe una sucesión de escalares $\left\{\lambda_{i, n}\right\}_{n \in \mathbb{N}}$ tal que

$$
\sum_{n \geq 1} \lambda_{i, n} y_{i, n} \text { converge y } \sum_{n=1}^{\infty}\left|\lambda_{i, n}\right|=\infty
$$

Podemos suponer $\lambda_{i, n} \in \mathbb{R}_{0}^{+}, \forall i, \forall n$, ya que basta tomar

$$
\left\{\begin{array}{l}
\hat{y}_{i, n}=\alpha_{i, n} y_{i, n} \\
\hat{y}_{i, n}^{*}=\alpha_{i, n}^{-1} y_{i, n}^{*}
\end{array}\right.
$$


donde $\alpha_{i, n}$ es un escalar de módulo uno haciendo cierta la igualdad $\alpha_{i, n}\left|\lambda_{i, n}\right|=\lambda_{i, n}$. Se conservan la ortogonalidad y la acotación y claramente sigue $\sin$ haber equivalencia con la base canónica de $l_{1}$ para cada $i \in I$. No es restrictivo tampoco imponer $\lambda_{i, 1}>0, \forall i \in I$. Definimos, para cada $i$ en $I$ y cada $n$ y $k$ naturales

$$
c_{i, n}^{k}= \begin{cases}\lambda_{i, n}\left(\sum_{j=1}^{k} \lambda_{i, j}\right)^{-1}, & \text { si } n \leq k \\ 0, & \text { si } n>k\end{cases}
$$

Entonces,

$$
c_{i, n}^{k} \geq 0, \sum_{n=1}^{\infty} c_{i, n}^{k}=1
$$

y, como consecuencia de (1),

$$
\lim _{k \rightarrow \infty}\left\|\sum_{n=1}^{\infty} c_{i, n}^{k} y_{i, n}\right\|=0
$$

Fijemos $\delta>0$ y $\left\{z_{i}: i \in I\right\}$ un subconjunto denso de $S_{X}$, y para cada $i \in I$ y cada $n \in \mathbb{N}$ tomemos

$$
x_{i, n}=\delta z_{i}+y_{i, n}
$$

Sea

$$
A=\overline{\operatorname{aco}}\left\{x_{i, n}: i \in I, n \in \mathbb{N}\right\} .
$$

De la elección de los vectores $x_{i, n}$ es obvio que

$$
A \subseteq(1+\delta) B_{X}
$$

Además, $\delta B_{X} \subseteq A$ : si $x \in S_{X}$ y $\eta>0$, podemos encontrar un índice $i$ tal que

$$
\left\|x-z_{i}\right\|<\frac{\eta}{2 \delta}
$$

La condición (2) nos proporciona $k \in \mathbb{N}$ de modo que

$$
\left\|\sum_{n=1}^{\infty} c_{i, n}^{k} y_{i, n}\right\|<\eta / 2
$$


entonces,

$$
\left\|\delta z_{i}-\sum_{n=1}^{\infty} c_{i, n}^{k} x_{i, n}\right\|=\left\|\sum_{n=1}^{\infty} c_{i, n}^{k} y_{i, n}\right\|<\eta / 2
$$

lo que unido a (3) nos dice finalmente que

$$
\left\|\delta x-\sum_{n=1}^{\infty} c_{i, n}^{k} x_{i, n}\right\|<\eta
$$

$y$, por tanto, $\delta x \in A$. Como $A$ es equilibrado, se tiene

$$
\delta B_{X} \subseteq A .
$$

Así pues, el funcional de Minkowski de $A$, define una norma en $X$, que notaremos $\|$. III, equivalente a la de partida. Como

$$
\left|y_{i, n}^{*}\left(x_{i, n}\right)\right| \geq 1-\delta(4+\varepsilon), \quad \forall i \in I, \quad \forall n \in \mathbb{N},
$$

imponiendo $\delta<\frac{1}{4+\varepsilon}$ queda bien definido el funcional

$$
x_{i, n}^{*}:=\frac{y_{i, n}^{*}}{y_{i, n}^{*}\left(x_{i, n}\right)}(i \in I, n \in \mathbb{N})
$$

Entonces, obviamente

$$
x_{i, n}^{*}\left(x_{i, n}\right)=1, \quad \forall i \in I, \quad \forall n \in \mathbb{N}
$$

y si $(i, n) \neq(j, m)$, por ser $y_{i, n}^{*}\left(y_{j, m}\right)=0$,

$$
\left|x_{i, n}^{*}\left(x_{j, m}\right)\right|=\frac{\left|y_{i, n}^{*}\left(\delta z_{j}+y_{j, m}\right)\right|}{\left|y_{i, n}^{*}\left(x_{i, n}\right)\right|}<\frac{(4+\varepsilon) \delta}{1-\delta(4+\varepsilon)} .
$$

Eligiendo convenientemente $\varepsilon$ y $\delta$ podemos conseguir que

$$
\frac{(4+\varepsilon) \varepsilon}{1-\delta(4+\varepsilon)}<\lambda
$$


Claramente

$$
\left\|x_{i, n}\right\|=1=\left\|x_{i, n}^{*}\right\|, \quad \forall(i, n) \in I \times \mathbb{N},
$$

$y$, por tanto, $(X,\|\cdot\| \|)$ verifica la propiedad $\alpha$ para la constante $\lambda$.

La demostración que nosotros hemos dado del teorema no es la original de W. Schachermayer ([34]), que hace uso del Teorema de Dvoretzky. Formalmente, es esencialmente la que se encuentra en [15, Theorem 1].

Si se analiza la demostración anterior, puede observarse que, si prescindimos de la elección de la constante $\lambda$ de la Definición 3.19, la distancia de Banach-Mazur entre los espacios $(X,\|\|$.$) y (X,\|\|$.$) puede$ conseguirse $K>9$. Surge de forma natural la cuestión: ¿Puede mejorarse la distancia de Banach-Mazur entre el espacio y su renormación con la propiedad $\alpha$ ?

A partir del teorema anterior, usando que los espacio W.C.G. admiten resoluciones proyectivas de la identidad (véase [9, Theorem VI.2.5]), y el hecho de que la propiedad $\alpha$ es suficiente para $A$, se obtiene:

3.35 Corolario. Todo espacio de Banach W.C.G. puede ser renormado equivalentemente con la propiedad $A$.

S. Shelah ([37]) probó la existencia de un espacio de Banach que no es isomorfo a un espacio con la propiedad $\alpha$, pero esto no resuelve el siguiente:

3.36 Problema. ¿Hay algún espacio de Banach de forma que con ninguna de sus normas equivalentes verifique la propiedad $A$ ? 


\section{Capítulo 4}

\section{Operadores que alcanzan su norma y la propiedad de Radon-Nikodym.}

En este capítulo vamos a estudiar la relación existente entre la propiedad de Radon-Nikodym (R.N.P.) y el problema de la densidad de los operadores que alcanzan la norma. En primer lugar, incluimos un resultado que nos asegura que la R.N.P. es una condición suficiente para la propiedad $A$ de Lindenstrauss. La primera demostración de este teorema se debe a J. Bourgain; en cambio, la que ofrecemos en esta memoria utiliza un principio de optimización perturbada, establecido por C. Stegall, principio que generaliza otro debido a J. Bourgain. Finalmente, presentamos un trabajo de $\mathbf{R}$. Huff en el que, usando esencialmente el mismo tipo de ideas, probó que un espacio que verifica la propiedad $A$ para todas sus normas equivalentes ha de tener la R.N.P. 


\section{A. El principio de optimización no lineal de Bourgain-Stegall.}

Para enunciar el principio de optimización al que aludíamos antes, necesitamos recordar algunos conceptos:

4.1 Definición. Sea $\Omega$ un espacio topológico de Hausdorff y $f: \Omega \longrightarrow \mathbf{R}$ una función. Decimos que $f$ expone fuertemente $a \Omega$ si existe un elemento $\omega_{0} \in \Omega$ tal que

(a) $f(\omega) \leq f\left(\omega_{0}\right), \forall \omega \in \Omega$.

(b) $\operatorname{Si}\left\{\omega_{n}\right\}_{n \in \mathbb{N}}$ es una sucesión de elementos de $\Omega$ de modo que $\left\{f\left(\omega_{n}\right)\right\}_{n \in \mathbb{N}}$ converge a $f\left(\omega_{0}\right)$, entonces la sucesión $\left\{\omega_{n}\right\}_{n \in N}$ converge a $\omega_{0}$.

También se dice que $f$ expone fuertemente $a \Omega$ en el punto $\omega_{0}$, en caso de que se tenga la situación antes descrita.

Es claro que si $f$ expone a $\Omega$, entonces $f$ alcanza su máximo en un único elemento de $\Omega$.

4.2 Ejemplo. Si $x_{0}$ es un punto de la esfera unidad de un espacio de Banach uniformemente convexo y $x^{*} \in S_{X}$. verifica que $x^{*}\left(x_{0}\right)=1$, entonces es inmediato comprobar que $\operatorname{Re} x^{*}$ expone fuertemente a $B_{X}$ en $x_{0}$. 
4.3 Definición. Si $\Omega$ es un espacio topológicc de Hausdorff y $f: \Omega \rightarrow \mathbb{R}$ una función mayorada. la serción de $\Omega$ determinada por $f y \alpha>0$ es el subconjunto $S(f, \Omega, \alpha) \subseteq \Omega$ dado por

$$
S(f, \Omega, \alpha)=\{\omega \in \Omega: f(\omega)>\sup f(\Omega)-\alpha\} .
$$

Nótese que si $(E, d)$ es un espacio métrico completo y $f: E \longrightarrow \mathbb{R}$ es una función mayorada y superiormente semicontinua ( $f$ es inferiormente semicontinua), entonces $f$ expone fuertemente a $E$ si, y sólo si,

$$
\lim _{\alpha \rightarrow 0} \operatorname{diam} S(f, E, \alpha)=0
$$

4.4 Definición ([11, Definition V.3.3]). Sea $C$ un subconjunto no vacío de un espacio de Banach $X . C$ es dentable si para cada número real positivo $\varepsilon>0$, existe $x \in C$ tal que

$$
x \notin \overline{\mathrm{co}}\left(C \backslash\left(x+\varepsilon B_{X}\right)\right) .
$$

4.5 Ejemplo. Cualquier bola de un espacio uniformemente convexo es dentable.

4.6 Lema. Un subconjunto no vacio $C$ de un espacio de Banach $X$ es dentable si, y sólo $3 i$, para cada $\varepsilon>0$ existen $x^{*} \in X^{*}$ y $\alpha>0$ tales que

$$
\operatorname{diam} S\left(\operatorname{Re} x^{*}, C, \alpha\right)<\varepsilon .
$$


Demostración. Supongamos en primer lugar que $C$ es dentable. Dado $\varepsilon>0$, existe $x \in C$ de forma que $x \notin \overline{\mathrm{co}}\left(C \backslash\left(x+(\varepsilon / 3) B_{X}\right)\right)$. Aplicando el Teorema de separación de conjuntos convexos, hay un funcional $x^{*} \in X^{*}$ y un número real positivo $\alpha$ verificando la igualdad

$$
\operatorname{Re} x^{*}(x)=\sup \operatorname{Re} x^{*}\left(C \backslash\left(x+(\varepsilon / 3) B_{X}\right)\right)+\alpha .
$$

Entonces,

$$
S\left(\operatorname{Re} x^{*}, C, \alpha\right) \subseteq x+(\varepsilon / 3) B_{X},
$$

Ja que si $y \in C \backslash\left(x+(\varepsilon / 3) B_{X}\right)$,

$$
\begin{gathered}
\operatorname{Re} x^{*}(y) \leq \sup \operatorname{Re} x^{*}\left(C \backslash\left(x+(\varepsilon / 3) B_{X}\right)\right)= \\
=\operatorname{Re} x^{*}(x)-\alpha \leq \sup \operatorname{Re} x^{*}(C)-\alpha,
\end{gathered}
$$

y, por tantc, $y \notin S\left(\operatorname{Re} x^{*}, C, \alpha\right)$. Así pues, diam $S\left(\operatorname{Re} x^{*}, C, \alpha\right)<\varepsilon$.

$\mathrm{Y}$ recíprocamente; sea $\varepsilon>0$. Por hipótesis

$$
\exists x^{*} \in X^{*}, \exists \alpha>0: \operatorname{diam} S\left(\operatorname{Re} x^{*}, C, \alpha\right)<\varepsilon .
$$

Elijamos $x \in S\left(\operatorname{Re} x^{*}, C, \alpha\right)$; er tal caso, $\left.x \notin \overline{\mathbf{c o}}\left(C \backslash x+\varepsilon B_{X}\right)\right)$, puesto que si $x \in \overline{\mathrm{co}}\left(C \backslash\left(x+\varepsilon B_{X}\right)\right)$, entonces

$$
\text { sup } \operatorname{Re} x^{*}(C)-\alpha<\operatorname{Re} x^{*}(x) \leq \sup \operatorname{Re} x^{*}\left(C \backslash\left(x+\varepsilon B_{X}\right)\right)
$$

y

$$
\sup \operatorname{Re} x^{*}(C)-\sup \operatorname{Re} x^{*}\left(C \backslash\left(x+\varepsilon B_{X}\right)\right)<\iota,
$$

luego existe $y \in C \backslash\left(x+\varepsilon B_{X}\right)$ de forma que

$$
\sup \operatorname{Re} x^{*}(C)-\operatorname{Re} x^{*}(y)<\alpha,
$$

esto es, $y \in S\left(\operatorname{Re} x^{*}, C, \alpha\right)$, lo que contradice (1), ya que $\|x-y\| \geq \varepsilon$. Por tanto,

$$
\left.x \notin \overline{\mathrm{co}}\left(C \backslash x+\varepsilon B_{X}\right)\right),
$$

de donde $C$ es no dentable. 
4.7 Definición. Un subconjunto $C$ de un espacio de Banach $X$ tiene la propiedad de Radon-Nikodym (R.N.P.) si todo subconjunto no vacío y acotado de $C$ es dentable.

Nótese que un espacio de Banach $X$ tiene la R.N.P. si, y sólo si, $B_{X}$ la tiene, ya que si un conjunto es dentable, su imagen por cualquier traslación o cualquier homotecia sigue siendo dentable.

Aquí hemos adoptado como definición de la R.N.P. una de las caracterizaciones geométricas. No pretendemos aquí, ni es objeto de este trabajo, hacer un estudio detallado de esta propiedad.

Probemos ahora que un espacio de Banach verifica la R.N.P. si, y sólo si, todo subconjunto no vacío, acotado, cerrado y absolutamente convexo es dentable. Para comprobar este hecho, si notamos por $\mathbb{T}$ a la esfera unidad del cuerpo, dado un subconjunto $C \subseteq X$ no vacío y acotado y $x^{*} \in X^{*}$, es claro que la función

$$
\begin{gathered}
\mathbb{T} \longrightarrow \mathbb{R} \\
x \mapsto \sup \operatorname{Re} x^{*}(\lambda C)
\end{gathered}
$$

es continua. Por tanto, existe $\lambda_{0} \in \mathbb{T}$ tal que

$$
\sup \operatorname{Re} x^{*}(T C)=\sup \operatorname{Re} x^{*}\left(\lambda_{0} C\right) .
$$

Como $\overline{\operatorname{aco}}(C)=\overline{c o}(\mathbb{T} C)$ es un subconjunto no vacío, absolutamente convexo, cerrado y acotado de $X$, si todo conjunto con estas propiedades es dentable, entonces, dado $\varepsilon>0$ existen $x^{*} \in X^{*}$ y $\alpha>0$ de modo que

$$
\operatorname{diam} S\left(\operatorname{Re} x^{*}, \overline{\operatorname{co}}(\mathbb{T} C), \alpha\right)<\varepsilon .
$$

Como

$$
S\left(\operatorname{Re} x^{*}, \lambda_{0} C, \alpha\right) \subseteq S\left(\operatorname{Re} x^{*}, \overline{\mathrm{co}}(\mathbb{T} C), \alpha\right),
$$




$$
\lambda_{0} S\left(\operatorname{Re} \lambda_{0} x^{*}, C, \alpha\right)=S\left(\operatorname{Re} x^{*}, \lambda_{0} C, \alpha\right)
$$

y $\left|\lambda_{0}\right|=1$, entonces

$$
\operatorname{diam} S\left(\operatorname{Re} \lambda_{0} x^{*}, C, \alpha\right)=\operatorname{diam} S\left(\operatorname{Re} x^{*}, \lambda_{0} C, \alpha\right)<\varepsilon .
$$

Así pues, $C$ es dentable y, por tanto, $X$ tiene la R.N.P.

Nuestro principal objetivo en esta sección es el siguiente resultado.

4.8 Teorema (Principio de optimización no lineal de Bourgain-Stegall [38, Theorem 14]). Sea $X$ un espacio de Banach real, $C \subseteq X$ un subconjunto no vacio, convexo, cerrado y acotado, verificando la R.N.P. Si $f: C \longrightarrow \mathbb{R}$ es una función mayorada y superiormente semicontinua, entonces el conjunto

$$
\left\{x^{*}: f+x^{*} \text { expone fuertemente a } C\right\}
$$

es denso en $X^{*}$.

El teorema anterior es otro principio de optimización perturbada, como lo son el Principio Variacional de Ekeland y el Teorema de BronstedRockafellar. La novedad de este resultado reside en el hecho de que una función, en general no lineal, se perturba por un funcional lineal continuo, de forma que la suma de ambos alcanza el máximo.

La demostración que haremos aquí no es la original de C. Stegall ([38]), la cual utiliza de manera esencial un resultado de J. Bourgain ([8]) (formalmente el mismo del enuciado del Teorema 4.8 para el caso particular $f=0)$. Nosotros seguimos la que aparece en el texto de R.R. Phelps ([32]), que, directamente y usando las ideas de J. Bourgain para construir conjuntos no dentables, permite demostrar el Teoreına 4.8 sin usar el resultado de J. Bourgain. 
El siguiente lema nos da un procedimiento para construir subconjuntos no dentables.

4.9 Lema ([8, Lemma 3]). Sea $\left\{A_{n}\right\}_{n \in N}$ una sucesión de subconjuntos de un espacio de Banach $X$ no vacios satisfaciendo la siguiente condición: existen números reales $\varepsilon>0$ y $\lambda>0$ de modo que si $x \in \operatorname{co}\left(A_{n}\right)$ e $y \in X$, se tiene que

$$
\operatorname{dist}\left(x, \operatorname{co}\left(A_{n+1} \backslash\left(y+\varepsilon B_{X}\right)\right)\right) \leq \lambda / 2^{n} .
$$

Entonces, el conjunto

$$
A=\bigcap_{n \in N} \overline{\bigcup_{j \geq n} \operatorname{co}\left(A_{j}\right)}
$$

es no vacío y no dentable.

Demostración. Vamos a probar inicialmente que $A$ es no vacío y que se verifica

$$
\operatorname{co}\left(A_{n}\right) \subseteq A+\frac{4 \lambda}{2^{n}} B_{X}, \forall n \in \mathbb{N}
$$

En efecto: sea $x_{0} \in \operatorname{co}\left(A_{n}\right)$. La condición impuesta a la sucesión $\left\{A_{n}\right\}_{n \in \mathrm{N}}$ nos da un elemento $x_{1} \in \operatorname{co}\left(A_{n+1}\right)$ tal que

$$
\left\|x_{0}-x_{1}\right\| \leq \frac{2 \lambda}{2^{n}}
$$

Por recurrencia, repitiendo el mismo procedimiento, construimos una sucesión $\left\{x_{k}\right\}_{k \in N}$ de modo que

$$
x_{k} \in \operatorname{co}\left(A_{n+k}\right) \text { y }\left\|x_{k}-x_{k+1}\right\| \leq \frac{2 \lambda}{2^{n+k}}, k=0,1, \ldots
$$

En particular, la serie $\sum_{k \geq 0}\left(x_{k}-x_{k+1}\right)$ converge a cierto vector $y \in X$, con $\|y\| \leq 4 \lambda / 2^{n}$, y obviamente, $y=x_{0}-z$, con $z=\lim _{k \rightarrow \infty} x_{k}$. Es evidente 
que $z \in A$ (con lo que $A$ es no vacío) y, por tanto,

$$
x_{0}=z+y \in A+\frac{4 \lambda}{2^{n}} B_{X}
$$

Para probar que $A$ no es dentable, comprobemos que si $x$ está en $A$, entonces

$$
x \in \overline{\mathrm{co}}\left(A \backslash\left(x+\frac{\varepsilon}{2} B_{X}\right)\right),
$$

siendo $\varepsilon>0$ el número real positivo que verifica la hipótesis. Dado un número real positivo $\eta$, elegimos un natural $n$ tal que

$$
\frac{5 \lambda}{2^{n}}<\min \left\{\frac{\varepsilon}{2}, \eta\right\}
$$

Como $x \in A$, existen un natural $j \geq n$ y un elemento $y \in \operatorname{co}\left(A_{j}\right)$ tales que

$$
\|x-y\| \leq \frac{\lambda}{2^{n}}
$$

Por hipótesis se tiene

$$
\operatorname{dist}\left(y, \operatorname{co}\left(A_{j+1} \backslash\left(x+\varepsilon B_{X}\right)\right) \leq \frac{\lambda}{2^{j}} \leq \frac{\lambda}{2^{n}},\right.
$$

luego existe $z \in \operatorname{co}\left(A_{j+1} \backslash\left(x+\varepsilon B_{X}\right)\right)$ de modo que $\|y-z\| \leq 2 \lambda / 2^{n}$. El elemento $z$ se puede expresar entonces de la forma

$$
z=\sum_{i=1}^{r} t_{i} u_{i}
$$

para un cierto natural $r$, reales positivos $t_{i}$, tales que $\sum_{i=1}^{r} t_{i}=1 \mathrm{y}$ elementos $u_{i} \in A_{j+1} \backslash\left(x+\varepsilon B_{X}\right)$. De (1), para cada $i$,

$$
u_{i} \in A+\frac{4 \lambda}{2^{j+1}} B_{X} \subseteq A+\frac{2 \lambda}{2^{n}} B_{X},
$$


por lo que, para cada $i$, existe $v_{i} \in A$ tal que $\left\|u_{i}-v_{i}\right\| \leq 2 \lambda / 2^{n}$. Si ponemos

$$
w=\sum_{i=1}^{r} t_{i} v_{i}
$$

se tiene $\|z-w\| \leq 2 \lambda / 2^{n}$, de donde, usando (2) y la elección de $z$ se tiene

$$
\begin{gathered}
\|x-w\| \leq\|x-y\|+\|y-z\|+\|z-w\| \leq \\
\leq \frac{\lambda}{2^{n}}+\frac{2 \lambda}{2^{n}}+\frac{2 \lambda}{2^{n}}=\frac{5 \lambda}{2^{n}}<\eta .
\end{gathered}
$$

Además, para cada $i$,

$$
\left\|v_{i}-x\right\| \geq\left\|u_{i}-x\right\|-\left\|u_{i}-v_{i}\right\| \geq \varepsilon-2 \lambda / 2^{n} \geq \varepsilon / 2,
$$

de donde

$$
w \in \operatorname{co}\left(A \backslash\left(x+\frac{\varepsilon}{2} B_{X}\right)\right)
$$

y por ser $\eta$ arbitrario, $x \in \overline{c o}\left(A \backslash\left(x+\frac{\varepsilon}{2} B_{X}\right)\right)$.

A continuación presentamos un resultado técnico útil en lo que sigue.

4.10 Lema. Sea $X$ un espacio de Banach real y $C$ un subconjunto no vacio tal que existe $M>0$ con $C \subseteq M B_{X}$. Entonces, si $f: C \longrightarrow \mathbb{R}$ es una función mayorada, para cada $\bar{\beta}>0$,

$$
S\left(f+x^{*}, C, \alpha\right) \subseteq S(f, C, \beta),
$$

siempre que $\left\|x^{*}\right\|<\beta / 2 M$ y $0<\alpha<\beta-2 M\left\|x^{*}\right\|$.

Demostración. Dado $x \in S\left(f+x^{*}, C, \alpha\right)$, se tiene

$$
f(x)+x^{*}(x)>\sup \left(f+x^{*}\right)(C)-\alpha>
$$




$$
\begin{gathered}
>\sup \left(f+x^{*}\right)(C)-\beta+2 M\left\|x^{*}\right\| \geq \\
\geq \sup f(C)+\inf x^{*}(C)-\beta+2 M\left\|x^{*}\right\| \geq \\
\geq \sup f(C)-\beta+x^{*}(x)
\end{gathered}
$$

y $x \in S(f, C, \beta)$.

El siguiente lema va a permitirnos simplificar la demostración del Teorema 4.8: bastará con perturbar la función mediante un funcional próximo a cero, de forma que esta perturbación determine una sección de diámetro pequeño.

4.11 Lema ([32, Lemma 5.19]). Sea $C$ un subconjunto no vacío, cerrado y acotado de un espacio de Banach real $X$, de forma que para toda función $g: C \longrightarrow \mathbb{R}$ superiormente semicontinua y mayorada, y todo $\eta>0$, existen $x^{*} \in X^{*} y \alpha>0$ verificando

$$
\left\|x^{*}\right\|<\eta, \operatorname{diam} S\left(g+x^{*}, C, \alpha\right) \leq 2 \eta .
$$

Entonces, si $f: C \longrightarrow \mathbb{R}$ es una tal función, para cada $\varepsilon>0$ existe $x^{*} \in X^{*}$ tal que $\left\|x^{*}\right\|<\varepsilon$ y $f+x^{*}$ expone fuertemente a $C$.

Demostración. Podemos suponer sin pérdida de generalidad que $C \subseteq B_{X}$ y $0<\varepsilon<1$. Por hipótesis, existen $x_{1}^{*} \in X^{*}$ y $0<\alpha_{1}<1$ de forma que

$$
\left\|x_{1}^{*}\right\|<\varepsilon / 2 \text { y } \operatorname{diam} S\left(f+x_{1}^{*}, C, \alpha_{1}\right) \leq \varepsilon .
$$

Aplicamos la hipótesis a $f+x_{1}^{*}$ y $\eta_{1}:=\varepsilon \alpha_{1} / 2^{2}$ y obtenemos $x_{2}^{*} \in X^{*}$ y $0<\alpha_{2}<\alpha_{1}$ tales que

$$
\left\|x_{2}^{*}\right\|<\eta_{1} \quad \text { y } \operatorname{diam} S\left(f+x_{1}^{*}+x_{2}^{*}, C, \alpha_{2}\right) \leq 2 \eta_{1} .
$$


Inductivamente, definimos tres sucesiones $\left\{\eta_{n}\right\}_{n \in \mathbb{N}}$ en $\mathbb{R}^{+},\left\{\alpha_{n}\right\}_{n \in \mathbb{N}}$ en ] $0,1\left[y\left\{x_{n}^{*}\right\}_{n \in N}\right.$ en $X^{*}$ de modo que

$$
\eta_{n}=\frac{\varepsilon \alpha_{n}}{2^{n+1}},\left\|x_{n}^{*}\right\|<\eta_{n-1}
$$

$$
\operatorname{diam} S\left(f+\sum_{i=1}^{n} x_{i}^{*}, C, \alpha_{n}\right) \leq 2 \eta_{n-1} \text { y } \alpha_{n}<\alpha_{n-1}, \quad \forall n \in \mathbb{N} \backslash\{1\}
$$

Por tanto, la serie $\sum_{n \geq 1} x_{n}^{*}$ converge en norma hacia un elemento $x^{*} \in X^{*}$, con

$$
\left\|x^{*}\right\| \leq \varepsilon \sum_{n=0}^{\infty} \frac{\alpha_{n}}{2^{n+1}}<\varepsilon
$$

Probemos finalmente que $f+x^{*}$ expone fuertemente a $C$. Para ello basta con demostrar que

$$
\lim _{\alpha \rightarrow 0} \operatorname{diam} S\left(f+x^{*}, C, \alpha\right)=0 .
$$

Puesto que de (1) tenemos que

$$
\lim _{n \rightarrow \infty} \operatorname{diam} S\left(f+\sum_{i=1}^{n} x_{i}^{*}, C, \alpha_{n}\right)=0
$$

es suficiente con verificar que, dado $n$ natural, existe $\alpha>0$ tal que $S\left(f+x^{*}, C, \alpha\right) \subseteq S\left(f+\sum_{i=1}^{n} x_{i}^{*}, C, \alpha_{n}\right)$. Para ello, hacemos uso del Lema 4.10 poniendo

$$
f+x^{*}=f+\sum_{i=1}^{n} x_{i}^{*}+s_{n}^{*}
$$

donde $s_{n}^{*}=\sum_{i=n+1}^{\infty} x_{i}^{*} \mathrm{y}$

$$
\left\|s_{n}^{*}\right\| \leq \sum_{i=n}^{\infty} \varepsilon \frac{\alpha_{i}}{2^{i+1}}<\frac{\alpha_{n}}{2} .
$$

Tomando $0<\alpha<\alpha_{n}-2\left\|s_{n}^{*}\right\|$ bastará usar el Lema 4.10 para concluir la demostración. 
Demostración del Teorema 4.8. Si $z^{*} \in X^{*}$ entonces $f+z^{*}$ está en las mismas condiciones que la función $f$, por lo que en vista del lema anterior, basta probar

$$
\forall \varepsilon>0, \quad \exists x^{*} \in X^{*}, \alpha>0:\left\|x^{*}\right\|<\varepsilon y \operatorname{diam} S\left(f+x^{*}, C, \alpha\right) \leq 2 \varepsilon .
$$

Supongamos que existe $\varepsilon>0$ tal que para cada funcional $x^{*}$ con $\left\|x^{*}\right\|<\varepsilon$ $y$ cada $\alpha>0$ se tiene que

$$
\operatorname{diam} S\left(f+x^{*}, C, \alpha\right)>2 \varepsilon .
$$

El Lema 4.9 nos va a proporcionar un subconjunto no dentable de $C$, en clara contradicción con el hecho de que $C$ tiene la R.N.P. Dado $n \in \mathbb{N}$, definimos

$$
A_{n}=\bigcup_{\left\|x^{*}\right\| \leq \varepsilon-1 / 2^{n}} S\left(f+x^{*}, C, 1 / 4^{n}\right)
$$

A partir de cierto natural, se tendrá $\varepsilon-1 / 2^{n}>0$, con lo que $A_{n} \neq \emptyset$. Para ponernos en las hipótesis del Lema 4.9 , podemos suponer, sin pérdida de generalidad alguna, que para cada natural $n, A_{n} \neq \emptyset$. Hay que probar que existen constantes $\varepsilon, \lambda>0$ tales que para cada natural $n$ se tiene

$$
\operatorname{co}\left(A_{n}\right) \subseteq \operatorname{co}\left(A_{n+1} \backslash\left(y+\varepsilon B_{X}\right)\right)+\frac{\lambda}{2^{n}} B_{X}, \quad \forall y \in X,
$$

y por ser el conjunto que aparece a la derecha convexo, bastará comprobar que

$$
A_{n} \subseteq \operatorname{co}\left(A_{n+1} \backslash\left(y+\varepsilon B_{X}\right)\right)+\frac{\lambda}{2^{n}} B_{X}, \forall y \in X
$$

Procediendo nuevamente por redución al absurdo, supongamos que hay un elemento $x \in A_{n}$ que no está en $\operatorname{co}\left(A_{n+1} \backslash\left(y+\varepsilon B_{X}\right)\right)+\left(\lambda / 2^{n}\right) B_{X}$, para algún $y \in X$. Por el Teorema de separación de conjuntos convexos, existe un funcional $y^{*} \in S_{X}$. tal que

$$
y^{*}(x) \geq \sup y^{*}\left(A_{n+1} \backslash\left(y+\varepsilon B_{X}\right)\right)+\frac{\lambda}{2^{n}} .
$$


La definición de $A_{n}$ asegura la existencia de un funcional $x^{*} \in X^{*}$, con $\left\|x^{*}\right\| \leq \varepsilon-1 / 2^{n}$, verificando

$$
x \in S\left(f+x^{*}, C, 1 / 4^{n}\right) .
$$

Si definimos $z^{*}:=x^{*}+1 / 2^{n+1} y^{*}$, entonces

$$
\left\|z^{*}\right\| \leq \varepsilon-\frac{1}{2^{n}}+\frac{1}{2^{n+1}}=\varepsilon-\frac{1}{2^{n+1}}
$$

y por tanto

$$
S\left(f+z^{*}, C, 1 / 4^{n+1}\right) \subseteq A_{n+1} .
$$

Pero en virtud de (1), el conjunto $S\left(f+z^{*}, C, 1 / 4^{n+1}\right)$ tiene diámetro mayor que $2 \varepsilon$, luego no está contenido en $y+\varepsilon B_{X}$. Sea entorices $z \in C \backslash\left(y+\varepsilon B_{X}\right)$ de forma que

$$
f(z)+z^{*}(z)>\sup \left(f+z^{*}\right)(C)-\frac{1}{4^{n+1}}
$$

Así, $z \in A_{n+1} \backslash\left(y+\varepsilon B_{X}\right)$ y de (3) se sigue

$$
y^{*}(x) \geq y^{*}(z)+\frac{\lambda}{2^{n}}
$$

Jeamos que esto no es posible, lo que nos dirá que (2) es cierto y por tanto también la tesis del teorema. Como por (4) $x \in S\left(f+x^{*}, C, 1 / 4^{n}\right)$, y $z \in C$, entonces

$$
f(x)+x^{*}(x)>f(z)+x^{*}(z)-1 / 4^{n}
$$

y de la desigualdad (5) y la definición de $z^{*}$ se deduce

$$
\begin{gathered}
f(z)+z^{*}(z)>\sup \left(f+z^{*}\right)(C)-\frac{1}{4^{n+1}}= \\
=\sup \left(f+x^{*}+\frac{1}{2^{n+1}} y^{*}\right)(C)-\frac{1}{4^{n+1}} \geq
\end{gathered}
$$




$$
\geq f(x)+x^{*}(x)+\frac{1}{2^{n+1}} y^{*}(x)-\frac{1}{4^{n+1}} .
$$

Usando las desigualdades (7) y (8) obtenemos ahora

$$
\begin{gathered}
f(z)+x^{*}(z)+\frac{1}{2^{n+1}} y^{*}(z)=f(z)+z^{*}(z)> \\
>f(x)+x^{*}(x)+\frac{1}{2^{n+1}} y^{*}(x)-\frac{1}{4^{n+1}} \geq \\
\geq f(z)+x^{*}(z)-\frac{1}{4^{n}}+\frac{1}{2^{n+1}} y^{*}(x)-\frac{1}{4^{n+1}}
\end{gathered}
$$

de donde $y^{*}(x-z)<5 / 2^{n+1}$. Tomando $\lambda=5 / 2$ se tiene

$$
y^{*}(x-z)<\frac{\lambda}{2^{n}}
$$

lo que contradice (6). Por tanto, (2) siempre es cierto para $\varepsilon>0$ y $\lambda=5 / 2$ y el Lema 4.9 proporciona entonces un subconjunto de $C$ no dentable.

4.12 Corolario ([38, Theorem 15]). Sean $X$ e $Y$ dos espacios de Banach (reales o complejos) y $C \subseteq X$ un subconjunto no vacio, cerrado y absolutamente convexo que verifica la R.N.P. Dados $T \in L(X, Y)$ y $\varepsilon>0$, existe un operador $S \in L(X, Y)$ que alcanza el supremo de su norma en $C$, tal que $S-T$ es un operador de rango uno y $\|S-T\|<\varepsilon$.

Demostración. Definimos la función

$$
f: C \longrightarrow \mathbb{R}
$$

por

$$
f(x)=\|T x\| \quad(x \in C) .
$$


El Teorema 4.8 asegura la existencia de un funcional $x^{*} \in X^{*}$ con $\left\|x^{*}\right\|<\varepsilon$ tal que $f+\operatorname{Re} x^{*}$ expone fuertemente a $C$ en cierto elemento $x_{0} \in C$. Como $C$ es equilibrado, $\lambda x \in C$, para cualquier escalar $\lambda$ de módulo uno y $x$ elemento de $C$, de donde

$$
\|T x\|+\operatorname{Re} x^{*}(\lambda x) \leq\left\|T x_{0}\right\|+\operatorname{Re} x^{*}\left(x_{0}\right), \quad \forall \lambda \in \mathbb{T}, \quad \forall x \in C,
$$

y esto obviamente implica que

$$
\operatorname{Re} x^{*}\left(x_{0}\right)=\left|x^{*}\left(x_{0}\right)\right|
$$

y de hecho se tiene

$$
\|T x\|+\left|x^{*}(x)\right| \leq\left\|T x_{0}\right\|+\operatorname{Re} x^{*}\left(x_{0}\right), \forall x \in C .
$$

Si $T x_{0}=0$, elegimos un elemento $y_{0} \in S_{Y}$ cualquiera, y si $T x_{0} \neq 0$, tomamos

$$
y_{0}=\frac{T x_{0}}{\left\|T x_{0}\right\|}
$$

y definimos un operador $S \in L(X, Y)$ mediante

$$
S x=T x+x^{*}(x) y_{0} \quad(x \in X) .
$$

Como $(S-T)(x)=x^{*}(x) y_{0}$, es claro que la diferencia $S-T$ es un operador en $L(X, Y)$ de rango uno y $\|S-T\|=\left\|x^{*}\right\|<\varepsilon$. Si $x \in C$, entonces se tiene

$$
\|S x\| \leq\|T x\|+\left|x^{*}(x)\right| \leq\left\|T x_{0}\right\|+\left|x^{*}\left(x_{0}\right)\right|
$$

mientras que

$$
\left\|S x_{0}\right\|=\left\|T x_{0}+x^{*}\left(x_{0}\right) y_{0}\right\|=\left\|T x_{0}\right\|+x^{*}\left(x_{0}\right)=\left\|T x_{0}\right\|+\left|x^{*}\left(x_{0}\right)\right|,
$$

es decir, la función 


$$
\begin{gathered}
C \longrightarrow \mathbf{R} \\
x \mapsto\|S x\|
\end{gathered}
$$

alcanza su máximo en el punto $x_{0}$.

\section{B. El Teorema de Bourgain-Huff.}

Como consecuencia del Corolario 4.12, si $X$ es un espacio de Banach verificando la R.N.P., entonces $X$ verifica la propiedad $A$. Más aún, como la R.N.P. es trivialmente estable por isomorfismos, todo espacio isomorfo a $X$ verifica la propiedad $A$. Y recíprocamente, usando un resultado de Huff, vamos a probar que si todo espacio de Banach isomorfo a $X$ tiene la propiedad $A$, entonces $X$ tiene la R.N.P.

Comprobamos en primer lugar que la R.N.P. es una propiedad determinada por los subespacios separables:

4.13 I,ema ([7]). Si un espacio de Banach $X$ no verifica la R.N.P., entorices existe un subconjunto no vacio, acotado $y$ numerable que es no dentable. En consecuencia, $X$ tiene la R.N.P. si, y sólo si, todo subespacio cerrado y separable suyo también la satisface.

Demostración. Sea $X$ un espacio que no satisface la R.N.P.; existe, por tanto, $C \subseteq X$, subconjunto no vacio, acotado y no dentable, esto es,

$$
\exists \varepsilon>0: \forall x \in C, x \in \overline{c o}\left(\tilde{C} \backslash\left(x+\varepsilon B_{X}\right)\right) \text {. }
$$



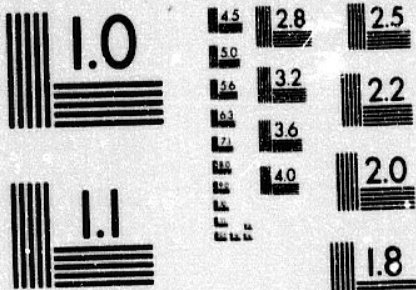

1. 3.6

4.0

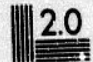

4

5.

는
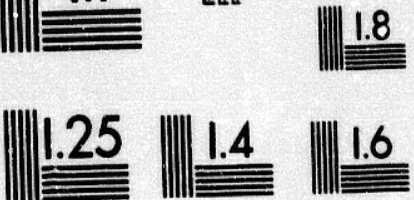

MICROCOPY RESOLUTION TEST CHART NATIONAL BUREAU OF STANDARDS STANDARD REFEAENCE MATERIAL 1010a (ANSI and ISO TEST CHART No. 2) 


$$
\begin{gathered}
C \longrightarrow \mathbb{R} \\
x \mapsto\|S x\|
\end{gathered}
$$

alcanza su máximo en el punto $x_{0}$.

\section{B. El Teorema de Bourgain-Huff.}

Como consecuencia del Corolario 4.12, si $X$ es un espacio de Banach verificando la R.N.P., entonces $X$ verifica la propiedad $A$. Más aún, como la R.N.P. es trivialmente estable por isomorfismos, todo espacio isomorfo a $X$ verifica la propiedad $A$. Y recíprocamente, usando un resultado de Huff, vamos a probar que si todo espacio ie Banach isomorfo a $X$ tiene la propiedad $A$, entonces $X$ tiene la R.N.P.

Comprobamos en primer lugar que la R.N.P. es una propiedad determinada por los subespacios separables:

4.13 Lema ([7]). Si un espacio de Banach $X$ no verifica la R.N.P., entonces existe un subconjunto no vacío, acotado $y$ numerable que es no dentable. En consecuencia, X tiene la R.N.P. si, y sólo si, todo subespacio cerrado y separable suyo también la satisface.

Demostración. Sea $X$ un espacio que no satisface la R.N.P.; existe, por tanto, $C \subseteq X$, subconjunto no vacío, acotado y no dentable, esto es,

$$
\exists \varepsilon>0: \forall x \in C, x \in \overline{\mathrm{co}}\left(C \backslash\left(x+\varepsilon B_{X}\right)\right) .
$$


Sea $x \in C$; la condición (1) garantiza la existencia de una sucesión $\left\{x_{n}\right\}_{n \in \mathbb{N}}$ de elementos de $C$ tal que para cada $n \in \mathbb{N}$ hay un natural $k_{n}$, un subconjunto $\left\{y_{i}^{n}: i=1,2, \ldots, k_{n}\right\}$ de $C \backslash\left(x+\varepsilon B_{X}\right)$ y un conjunto de números reales $\left\{t_{i}^{n}: i=1,2, \ldots, k_{n}\right\}$, con $0 \leq t_{i}^{n}, i=1,2, \ldots, k_{n} \mathrm{y}$ $\sum_{i=1}^{k_{n}} t_{i}^{n}=1$, verificando

$$
x_{n}=\sum_{i=1}^{k_{n}} t_{i}^{n} y_{i}^{n} \text { y } x=\lim _{n \rightarrow \infty} x_{n} .
$$

Definimos entonces

$$
A(x)=\cup_{n \in N}\left\{y_{i}^{n}: i=1,2, \ldots, k_{n}\right\} .
$$

Para cada $x \in C, A(x)$ es un subconjunto de $C$ verificando

$$
\left\{\begin{array}{l}
x \in \overline{c o}(A(x)) \\
A(x) \cap\left(x+\varepsilon B_{X}\right)=\emptyset \\
A(x) \text { es numerable }
\end{array}\right.
$$

Construimos ahora una sucesión de subconjuntos numerables de $C$, $\left\{C_{n}\right\}_{n \in \mathbb{N}}$, por recurrencia. Elegimos un elemento $x \in C$ y tomamos

$$
C_{1}=\{x\}
$$

Supuesto construido $C_{n}$, ponemos

$$
C_{n+1}=\bigcup_{y \in C_{n}} A(y)
$$

Entonces, el subconjunto de $C$ dado por

$$
C^{\prime}:=\bigcup_{n} C_{n}
$$

es un subconjunto de $X$ no vacío, acotado y numerable. Además, no es dentable, ya que si $x \in C^{\prime}$, existe un natural $n$ tal que $x \in C_{n}$. Entonces, de (2),

$$
x \in \overline{\mathrm{co}}(A(x)) \subseteq \overline{\mathrm{co}}\left(C^{\prime} \backslash\left(x+\varepsilon B_{X}\right)\right)
$$


En particular, el subespacio cerrado y separable de $X$

$$
X_{0}:=\varlimsup \operatorname{in}\left(C^{\prime}\right)
$$

no verifica la R.N.P.

Una caracterización de la R.N.P. obtenida por Davis y Phelps nos será útil:

4.14 Lema ([11, Theorem VII.4.2]). Un espacio de Banach verifica la R.N.P. si, y sólo si, toda norma equivalente a la suya tiene bola unidad dentable.

Demostración. Si $X$ es un espacio que no satisface la R.N.P., entonces existe un subconjunto $C$ del mismo cerrado, acotado y absolutamente convexo no dentable. Probemos que el conjunto $A:=B_{X}+C$ no es dentable, con lo que el funcional de Minkowski de $A$ será una norma en $X$, equivalente a la de partida, y con bola unidad no dentable. Podemos suponer, sin pérdida de generalidad, que $X$ es un espacio de Banach real. Dados $x^{*} \in X^{*}, \alpha>0$ y $x \in B_{X}$ con $x^{*}(x)>\left\|x^{*}\right\|-\alpha / 2$, comprobemos que

$$
S\left(x^{*}, A, \alpha\right) \supseteq x+S\left(x^{*}, C, \alpha / 2\right)
$$

en efecto, si $y \in S\left(x^{*}, C, \alpha / 2\right)$,

$$
\begin{aligned}
x^{*}(x+y) & >\left\|x^{*}\right\|-\frac{\alpha}{2}+\sup x^{*}(C)-\frac{\alpha}{2} \geq \\
& \geq \sup x^{*}\left(B_{X}+C\right)-\alpha,
\end{aligned}
$$

luego $x+y \in S\left(x^{*}, A, \alpha\right)$. Por tanto,

$$
\operatorname{diam} S\left(x^{*}, C, \alpha / 2\right) \leq \operatorname{diam} S\left(x^{*}, A, \alpha\right)
$$


y como $C$ no es dentable, no admite secciones de diámetro arbitrariamente pequeño, por lo que en vista de (1), tampoco $A$ las admite.

El recíproco es evidente.

Seguidamente establecemos un resultado válido en el ambiente de espacios norriados.

4.15 Lema. Sea $(X,\|\cdot\|)$ un espacio normado y $X_{0}$ un subespacio suyo que posee una norma $\|\cdot\| x_{0}$ equivalente a la de partida (i.e., la heredada de $(X,\|\cdot\|)$. Entonces, existe una norma $\|\cdot\|$ en $X$, equivalente a la inicial, tal que \|\|$\cdot \| X_{0}$ coincide con la norma inducida por $(X,\|\cdot\|)$ en $X_{0}$.

Demostración. Sean $B_{X}$ y $\tilde{B}_{X_{0}}$ los conjuntos definidos por

$$
B_{X}=\{x \in X:\|x\| \leq 1\}
$$

y

$$
\tilde{B}_{X_{0}}=\left\{z \in X_{0}:\|z\|_{X_{0}} \leq 1\right\} .
$$

Como el vector 0 es interior a $\tilde{B}_{X_{0}}$ y las normas son equivalentes, existe $\rho>0$ tal que

Definimos

$$
\rho B_{X} \cap X_{0} \subseteq \tilde{B}_{X_{0}}
$$

$$
A=\overline{c o}\left(\rho B_{X} \cup \tilde{B}_{X_{0}}\right) .
$$

El funcional de Minkowski de dicho conjunto $A$, define claramente una norma $\|\cdot\| \|$ en $X$ equivalente a la inicial. Además, veamos que

$$
\|x\|=\|x\|_{X_{0}}, \quad \forall x \in X_{0} .
$$

Como $\tilde{B}_{X_{0}} \subseteq A$, entonces $\|x\| \leq\|x\|_{X_{0}}, \forall x \in X_{0}$. Y recíprocamente, la condición (1) nos da que

$$
X_{0} \cap A \subseteq \tilde{B}_{X_{0}}
$$


Como $\tilde{B}_{X_{0}} \subseteq A$, entonces $\|x\| \leq\|x\| X_{X_{0}}, \forall x \in X_{0}$. Y recíprocanente, la condición (1) nos da que

$$
X_{0} \cap A \subseteq \tilde{B}_{X_{0}}
$$

de donde se obtiene la otra desigualdad.

El siguiente lema nos ofrece una caracterización de la dentabilidad para una amplia clase de conjuntos.

4.16 Lema ([20, Theorem 1]). Sea $C$ un subconjunto cerrado, convexo $y$ acotado de un espacio de Banach $X$. Son equivalentes las afirmaciones:

(i) $C$ no es dentable.

(ii) Existe $\varepsilon>0$ de forma que ninguna sección de $C$ admite un recubrimiento del tipo $F+\varepsilon B_{X}$, donde $F$ es un subconjunto finito de $X$.

(iii) Existe $\varepsilon>0$ tal que

$$
C=\overline{\mathrm{co}}\left(C \backslash\left(F+\varepsilon B_{X}\right)\right),
$$

cualquiera que sea $F$ subconjunto finito de $X$.

Demostración. Supondremos, sin pérdida de generalidad, que $X$ es un espacio de Banach real.

(i) $\Rightarrow$ (ii) Claramente podemos asumir que $C \subseteq B_{X}$. Por no ser $C$ dentable, en virtud del Lema 4.6, existe $\delta>0$ tal que

$$
\operatorname{diam} S\left(x^{*}, C, \alpha\right)>\delta, \forall x^{*} \in X^{*}, \forall \alpha>0 .
$$

Procedamos por reducción al absurdo. Sean $x^{*} \in X^{*}$ y $\alpha>0$ tales que

$$
S\left(x^{*}, C, \alpha\right) \subseteq F+\varepsilon B_{X},
$$


para cierto subconjunto finito $F=\left\{x_{1}, x_{2}, \ldots, x_{n}\right\}$ de $X$, siendo $\varepsilon=\delta / 3$. Podemos suponer obviamente que

$$
S\left(x^{*}, C, \alpha\right) \cap\left(\left\{x_{i}\right\}+\varepsilon B_{X}\right) \neq \emptyset, \quad \forall i=1,2, \ldots, n .
$$

Definimos

$$
S=\overline{S\left(x^{*}, C, \alpha\right)}=\left\{x \in C: x^{*}(x) \geq \sup x^{*}(C)-\alpha\right\}
$$

y

$$
H=\left\{x \in C: x^{*}(x)=a\right\},
$$

con $a=\sup x^{*}(C)-\alpha$. Por ser $H \neq S$, podemos determinar un natural $m \leq n$ verificando simultáneamente

$$
S=\overline{c o}\left[H \cup\left(S \cap\left(\left\{x_{1}, x_{2}, \ldots, x_{m}\right\}+\varepsilon B_{X}\right)\right)\right]
$$

y

$$
S \neq K_{1}:=\overline{c o}\left[H \cup\left(S \cap\left(\left\{x_{2}, x_{3}, \ldots, x_{m}\right\}+\varepsilon B_{X}\right)\right)\right]
$$

(si $m=1$, tomamos $K_{1}=H$ ). Elijamos $x_{0} \in\left(S \cap B\left(x_{1}, \varepsilon\right)\right) \backslash K_{1} \mathrm{e}$ $y^{*} \in X^{*}$ de modo que

$$
c:=\sup y^{*}(S) \geq y^{*}\left(x_{0}\right)>\sup y^{*}\left(K_{1}\right)=a
$$

(si $m \geq 2$, la condición (2) nos asegura que $K_{1}$ es no vacío y si $m=1$, $K_{1}=H$ tampoco es vacío, ya que si $H=\emptyset$,

$$
S=\overline{c o}\left(S \cap\left(\left\{x_{1}\right\}+\varepsilon B_{X}\right)\right)=S \cap\left(\left\{x_{1}\right\}+\varepsilon B_{X}\right)
$$

por lo que $S \subseteq\left\{x_{1}\right\}+\varepsilon B_{X}$ y, por tanto,

$$
\delta<\operatorname{diam} S \leq 2 \varepsilon=\frac{2 \delta}{3},
$$

lo cual es absurdo). Sea $\beta>0$ tal que

$$
a<\beta<c \text { y } \frac{\beta-a}{c-a}>1-\frac{\delta}{12} .
$$


Definimos entonces $b:=c-\beta>0 y$

$$
S^{\prime}=\overline{S\left(y^{*}, S, b\right)} \text {. }
$$

Probemos en primer lugar que $S^{\prime}$ tiene diámetro menor o igual que $\delta$. Para ello, consideremos los conjuntos

$$
L=\left\{x \in B\left(x_{1}, \varepsilon\right) \cap S: y^{*}(x) \geq a\right\}
$$

y

$$
K_{2}=\left\{x \in S: y^{*}(x) \leq a\right\}
$$

Como

$$
L \cup K_{2} \supseteq H \cup\left(S \cap\left\{x_{1}, x_{2}, \ldots, x_{m}\right\}+\varepsilon B_{X}\right),
$$

entonces,

$$
S=\overline{c o}\left(L \cup K_{2}\right) \text {. }
$$

Por tanto, y puesto que $S\left(y^{*}, S, b\right)$ es abierto relativo en la topología de $S^{\prime}$, que es, además denso en $S^{\prime}$ y $\operatorname{co}\left(L \cup K_{2}\right)$ es denso en $S$, se tiene

$$
S^{\prime}=\overline{S^{\prime} \cap \operatorname{co}\left(L \cup K_{2}\right)} \text {. }
$$

Si ahora $u \in S^{\prime} \cap \operatorname{co}\left(L \cup K_{2}\right)$, entonces existen $\lambda \in[0,1], x \in L, y \in K_{2}$ tales que $u=\lambda x+(1-\lambda) y$, luego, la definición de los conjuntos $S^{\prime}, L$ y $K$ nos da

$$
\beta \leq y^{*}(u) \leq y^{*}(\lambda x+(1-\lambda) y) \leq \lambda c+(1-\lambda) a,
$$

y de ahi, $\lambda>(\beta-a) /(c-a)>1-\delta / 12$. Así, si $\lambda x+(1-\lambda) y$, $t x^{\prime}+(1-t) y^{\prime} \in S^{\prime} \cap \operatorname{co}\left(L \cup K_{2}\right)$,

$$
\begin{gathered}
\left\|\lambda x+(1-\lambda) y-t x^{\prime}-(1-t) y^{\prime}\right\| \leq \\
\leq\left\|\lambda x-t x^{\prime}\right\|+\|(1-\lambda) y\|+\left\|(1-t) y^{\prime}\right\| \leq \\
\leq\left\|x-(1-\lambda) x-x^{\prime}+(1-t) x^{\prime}\right\|+\delta / 12+\delta / 12 \leq
\end{gathered}
$$




$$
\left(C \subseteq B_{X}, x, x^{\prime} \in L\right) \leq\left\|x-x^{\prime}\right\|+\frac{4 \delta}{12} \leq 2 \varepsilon+\frac{\delta}{3}=\delta .
$$

Por tanto, el diámetro de $S\left(y^{*}, S, b\right)$ coincide con el de $S^{\prime}$ y no supera a $\delta$, a la luz de (3) y (4). Pero de la no dentabilidad de $C$ (véase (1)) se sigue que $S\left(y^{*}, S, b\right)$ no contiene ninguna sección de $C$, luego

$$
y^{*}(z)>\sup y^{*}(C)-b \geq c-b=\beta
$$

y $z \notin S\left(y^{*}, S, P\right)$, lo que dice en particular que $z \notin S$, por lo que $x^{*}(z)<a$. La deînición de los conjuntos $S^{\prime}$ y $K_{1}$ nos da inmediatamente que $S^{\prime} \cap K_{1}=\emptyset$. Por tanto, si elegimos $v \in S^{\prime}, v$ no es un elemento de $K_{1}$, y menos aún de $H$, esto es, se verifica

$$
x^{*}(v)>a .
$$

Como teníamos garantizada la existencia de $z \in C$ tal que $x^{*}(z)<a$, entonces, para conveniente $\mu \in] 0,1[, \mu z+(1-\mu) v \in H$. Pero sabemos también que $y^{*}(\mu z+(1-\mu) v) \geq \beta$, luego

$$
\mu z+(1-\mu) v \in H \cap S^{\prime}
$$

Llegamos pues a que $H \cap S^{\prime} \neq \emptyset$ lo cual es contradictorio.

(ii) $\Rightarrow$ (iii) Supongamos que (iii) no es cierto. Sea $\varepsilon>0$; existe entonces un subconjunto finito $F$ de $X$ y un elemento $x \in C$ tales que

$$
x \notin \overline{\mathrm{co}}\left(C \backslash\left(F+\varepsilon B_{X}\right)\right) .
$$

En virtud del Teorema de separación de conjuntos convexos, existe $x^{*}$ en $X^{*}$ y $\alpha>0$ verificando

$$
x^{*}(x)=\sup x^{*}\left(C \backslash\left(F+\varepsilon B_{X}\right)\right)+\alpha .
$$

Entonces,

$$
S\left(x^{*}, C, \alpha\right) \subseteq F+\varepsilon B_{X}
$$


Así pues, dado $\varepsilon>0$, existe un subconjunto finito $F$ de $X, x^{*} \in X^{*} y$ $\alpha>0$ verificando (5), con lo que negamos la afirmación (ii).

(iii) $\Rightarrow$ (i) Es obvio.

El principal resultado de esta sección es el siguiente:

4.17 Teorema ([19]). Si un espacio de Banach $X$ no verifica la R.N.P., entonces hay dos normas en $X,\|\cdot\|_{1} y\left\|_{1}\right\|_{2}$, equivalentes a la de partida, tales que el conjunto $N A\left(\left(X,\|\cdot\|_{1}\right),\left(X,\|\cdot\|_{2}\right)\right)$ no es denso en el espacio $L\left(\left(X,\|\cdot\|_{1}\right),\left(X,\|\cdot\|_{2}\right)\right)$.

Demostración. Sea $X_{0}$ un subespacio cerrado y separable de $X$ sin la R.N.P. (Lema 4.13). Entonces, en virtud del Lema 4.14, hay una norma en $X_{0}$, equivalente a la de partida, de forma que su bola unidad no es dentable. Esta norma se extiende a una nueva norma en $X,\|\cdot\|$, equivalente a la inicial (Lema 4.15). La no dentabilidad de $B_{X_{0}}$ nos proporciona un $\varepsilon>0$ de modo que

$$
\frac{1}{2} B_{X_{0}}=\overline{c o}\left(\frac{1}{2} B_{X_{0}} \backslash\left(F+2 \varepsilon B_{X}\right)\right)
$$

cualquiera que sea $F$ subconjunto de $X$ finito (Lema 4.16). Definimos

$$
C:=\overline{\frac{1}{2} B_{X_{0}}+\frac{\varepsilon}{8} B_{X}}
$$

Es fácil comprobar que

$$
C=\overline{c o}\left(C \backslash\left(F+\varepsilon B_{X}\right)\right), \forall F \subseteq X, F \text { finito. }
$$

Sea $\left\{z_{n}: n=1,2, \ldots\right\}$ un subconjunto denso de $X_{0}$. Definimos $X_{n}:=\mathbb{K} z_{n}$ y la aplicación

$$
\varphi: X \rightarrow l_{2} \text { dada por }
$$




$$
\varphi(x)(n)= \begin{cases}\|x\|, & \text { si } n=0 \\ \frac{1}{2^{n}} \operatorname{dist}\left(x, X_{n}\right), & \text { si } n \geq 1\end{cases}
$$

Si ponemos

$$
\|x\|_{2}:=\|\varphi(x)\| \quad(x \in X),
$$

obtenemos una norma en $X$ equivalente a la de partida \|.\|. Ahora consideramos la identidad formal $I$ de $(X,\|\|$.$) en \left(X,\|.\|_{2}\right)$ y comprobamos que todo operador $T:(X,\|\cdot\|) \longrightarrow\left(X,\|\cdot\|_{2}\right)$ verificando $\|T-I\|<\varepsilon / 4$ cumple que la función

$$
x \mapsto\|T x\|_{2}
$$

no alcanza el supremo en $C$. La prueba se concluye tomando como $\|\cdot\|_{1}$ el funcional de Minkowski del conjunto $C$. Supongamos que existe $x_{0} \in C$ tal que

$$
\left\|T x_{0}\right\|_{2}=\sup \left\{\|T x\|_{2}: x \in C\right\} \text {. }
$$

Como $x_{0} \in C, \operatorname{dist}\left(x_{0}, X_{0}\right) \leq \varepsilon / 8$, luego

$$
\exists q \in \mathbb{N}:\left\|x_{0}-z_{q}\right\|<\varepsilon / 4 .
$$

Recubrimos $(1+\varepsilon) B_{X_{q}}$ por un número finito de bolas de radio $\varepsilon / 8$ :

$$
(1+\varepsilon) B_{X_{q}} \subseteq F+\varepsilon / 8
$$

Definimos

$$
D:=\left\{y \in C: \operatorname{dist}\left(y, X_{q}\right)<\frac{7 \varepsilon}{8}\right\} .
$$

Si $y \in D$, existe $x \in X_{q}$ de modo que $\|x-y\|<\frac{7 \epsilon}{8}$, pero entonces

$$
\|x\| \leq \frac{7 \varepsilon}{8}+\|y\|<1+\varepsilon
$$

y por tanto, de (3) se sigue la existencia de $z \in F$ tal que $\|x-z\| \leq \frac{\varepsilon}{8}$. Así pues, $y \in F+\varepsilon B_{X}$ y $D \subseteq F+\varepsilon B_{X}$, por lo que, usando (1), obtenemos

$$
C=\overline{\mathrm{co}}(C \backslash D),
$$


y de ahí

$$
2\left\|T x_{0}\right\|_{2}=\sup \left\{\left\|T x_{0}+T \omega\right\|_{2}: \omega \in C \backslash D\right\} .
$$

Además, si $w \in C \backslash D$,

$$
\left\|\varphi\left(T x_{0}+T w\right)\right\| \leq\left\|\varphi\left(T x_{0}\right)+\varphi(T w)\right\|,
$$

ya que

$$
\varphi(u+v)(n) \leq \varphi(u)(n)+\varphi(v)(n), \quad \forall u, v \in X, \quad n=0,1, \ldots
$$

Uniendo (4) y (5) se tiene

$$
\begin{gathered}
\sup \left\{\left\|\varphi\left(T x_{0}\right)+\varphi(T w)\right\|: w \in C \backslash D\right\} \geq \\
\geq \sup \left\{\left\|\varphi\left(T x_{0}+T w\right)\right\|: w \in C \backslash D\right\}=2\left\|\varphi\left(T x_{0}\right)\right\| .
\end{gathered}
$$

La identidad del paralelogramo, junto con el hecho de ser

$$
\|\varphi(T w)\| \leq\left\|\varphi\left(T x_{0}\right)\right\|, \quad \forall w \in C \backslash D
$$

nos dice que

$$
\begin{gathered}
\left\|\varphi\left(T x_{0}\right)+\varphi(T w)\right\|^{2}+\left\|\varphi\left(T x_{0}\right)-\varphi(T w)\right\|^{2} \leq \\
\leq 4\left\|\varphi\left(T x_{0}\right)\right\|^{2}, \quad \forall \omega \in C \backslash D
\end{gathered}
$$

luego de (6) y (7),

$$
\inf \left\{\left\|\varphi\left(T x_{0}\right)-\varphi(T w)\right\|^{2}: w \in C \backslash D\right\}=0
$$

Pero, en vista de (2),

$$
\operatorname{dist}\left(T x_{0}, X_{q}\right) \leq\left\|T x_{0}-x_{0}\right\|+\operatorname{dist}\left(x_{0}, X_{q}\right) \leq \frac{\varepsilon}{4}+\frac{\varepsilon}{4}=\frac{\varepsilon}{2},
$$

en tanto que para todo $w \in C \backslash D$,

$$
\operatorname{dist}\left(T w, X_{q}\right) \geq \operatorname{dist}\left(w, X_{q}\right)-\|T w-w\| \geq \frac{7 \varepsilon}{8}-\frac{\varepsilon}{4}=\frac{5 \varepsilon}{8}
$$


por lo que

$$
\begin{gathered}
\left\|\sum_{n=1}^{\infty} \frac{\left(\operatorname{dist}\left(T x_{0}, X_{n}\right)-\operatorname{dist}\left(T w, x_{n}\right)\right)^{2}}{4^{n}}+\right\| T x_{0}-T w \|^{2} \geq \\
\geq \frac{\left(\operatorname{dist}\left(T x_{0}, X_{q}\right)-\operatorname{dist}\left(T w, X_{q}\right)\right)^{2}}{4^{q}}>\frac{(\varepsilon / 8)^{2}}{4^{q}}
\end{gathered}
$$

lo que contradice (8).

Para enunciar algunas consecuencias del teorema, establezcamos previamente un nuevo concepto:

4.18 Definición ([11, Chapter VII §5]). Se dice que un subconjunto no vacío, convexo, cerrado y acotado $C$ de un espacio de Banach $X$ tiene la propiedad de Bishop-Phelps si para cada espacio de Banach $Y$, el conjunto de los operadores $T \in L(X, Y)$ tales que la función

$$
x \mapsto\|T x\|
$$

alcanza su máximo en $C$, es denso el $L(X, Y)$. Un espacio de Banach $X$ verifica la propiedad de Bishop-Phelps si todo subconjunto suyo no vacío, convexo, cerrado y acotado satisface dicha propiedad.

Así, es lo mismo decir que $X$ tiene la propiedad $A$ que su bola verifica la propiedad de Bishop-Phelps.

4.19 Corolario ([8, Corollary 2]). Todo espacio de Banach con la propiedad de Bishop-Phelps verifica la R.N.P. 
4.20 Corolario ([19, Corollary 2$])$. Si $X$ es un espacio de Banach tal que todo espacio isomorfo a $X$ tiene la propiedad $B$, entonces $X$ tiene la R.N.P.

El recíproco de este último resultado no es cierto (véase la sección 5.B del Capítulo V de esta memoria).

4.21 Corolario ([19, Corollary 1]). Sea $X$ un espacio de Banach de forma que todo espacio isomorfo a $X$ verifica la propiedad $A$. Entonces $X$ satisface la R.N.P.

Ya sabíamos, como consecuencia del Principio de optimización de Bourgain-Stegall, que el recíproco de esta afirmación también es cierto.

A modo de síntesis de los resultados expuestos en este capítulo presentamos el siguiente corolario.

4.22 Corolario. Sea $X$ un espacio de Banach. Equivalen las siguientes afirmaciones:

(i) X verifica la propiedad de Bishop-Phelps.

(ii) Todo espacio de Banach isomorfo a $X$ tiene la propiedad $A$.

(iii) X tiene la R.N.P.

Otro resultado interesante, enmarcable dentro de este capítulo y que no probaremos, es debido a J.J. Uhl. y caracteriza los espacios de Banach estrictamente convexos que verifican la R.N.P. en los siguientes términos. 
4.23 Teorema ([39, Theorem 1, Theorem 2]). Si $Y$ es un espacio de Banach estrictamente convexo, entonces el conjunto $N A\left(L_{1}[0,1], Y\right)$ es denso en el correspondiente espacio de operadores si, y sólo si, $Y$ tiene la R.N.P. 


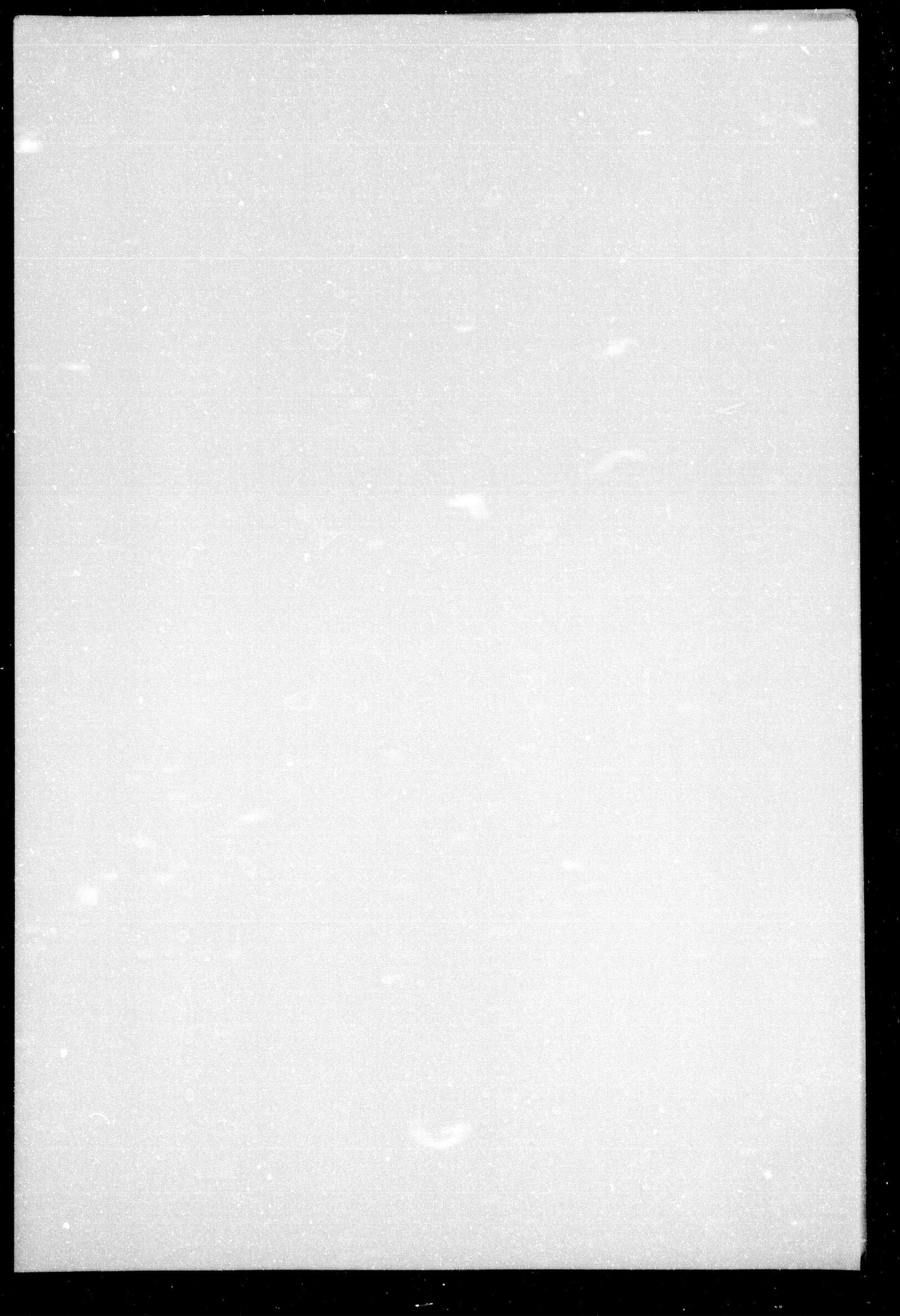




\section{Capítulo 5}

\section{Algunos resultados en espacios clásicos.}

Concluimos la presente memoria presentando un capítulo dedicado al estudio del problema de Bishop-Phelps, para ciertos espacios de Banach clásicos, así como para la clase de los operadores compactos entre dos espacios de Banach. En concreto, vamos a poner de manifiesto la densidad de los conjuntos $N A(C(K), C(S)), N A\left(C[0,1], L_{1}[0,1]\right)$ y $N A\left(L_{1}[0,1], L_{1}[0,1]\right)$ en los correspondientes espacios de operadores y la no densidad de $N A\left(L_{1}[0,1], C(S)\right)$ y $N A\left(G, l_{p}\right)$, con $(1<p<\infty)$, para convenientes espacio topológico compacto de Hausdorff $S$ y espacio de Banach $G$. Por último, se prueba que el conjunto de los operadores compactos entre dos espacios de Banach $X$ e $Y$ que alcanzan la norma es denso en el espacio de operadores compactos de $X$ en $Y$ siempre que $X$ o $Y$ sea un espacio del tipo $C(K)$ o $L_{1}(\mu)$. 


\section{A. Espacios $C(K)$ y $L_{1}(\mu)$.}

En esta primera sección probaremos en primer lugar dos resultados de J. Johnson y J. Wolfe. El primero, que apareció en un artículo de 1.979, asegura la densidad de los operadores que alcanzan la norma entre dos espacios de funciones continuas con valores reales, definidas en sendos espacios topológicos compactos y de Hausdorff ([24]). El segundo, publicado tres años más tarde, prueba la existencia de un espacio métrico compacto $S$ para el cual el conjunto $N A\left(L_{1}[0,1], C(S)\right)$ no es denso en $L\left(L_{1}[0,1], C\left(S^{\prime}\right)\right)$. Presentamos también una caracterización debida a $\mathrm{J}$. Lindenstrauss ([27]) de los espacios métricos compactos para los que el espacio $C(K)$ tiene la propiedad $A$ ( $K$ finito). Por último, daremos un resultado que es consecuencia de un trabajo de $W$. Schachermayer de 1.983 en el que se obtiene, en particular, la densidad del conjunto $N A\left(C[0,1], L_{1}[0,1]\right)$ en $L\left(C[0,1], L_{1}[0,1]\right)$.

Puesto que vamos a manejar los operadores con valores en un espacio del tipo $C(K)$ como funciones, nos será de utilidad la siguiente notación. Para un espacio topológico compacto y de Hausdorff $S$ y un espacio de Banach $X$, denotaremos por $\omega^{*}\left(S, X^{*}\right)$ el espacio de Banach de las aplicaciones $u: S \longrightarrow X^{*}$ que son continuas considerando en $X^{*}$ la topología $\omega^{*}$, dotado con la norma

$$
\|u\|=\sup \{\|u(s)\|: s \in S\} \quad\left(u \in \omega^{*}\left(S, X^{*}\right)\right) .
$$

(nótese que por ser $S$ un espacio topológico compacto y de Hausdorff y $u \omega^{*}$-continua, el conjunto $\{\|u(s)\|: s \in S\}$ está acotado).

$C(S)$ representará en esta sección el espacio de las funciones continuas en $S$ con valores reales.

La siguiente proposición, de demostración inmediata, nos permite representar los operadores de un espacio de Banach $X$ en el espacio de 
Banach $C(S)$ como funciones $\omega^{*}$-continuas.

5.1 Proposición ([13, Theorem VI.7.1]). Sea $S$ un espacio topológico compacto $y$ de Hausdorff y $X$ un espacio de Banach. Entonces, la aplicación

$$
\Phi: L(X, C(S)) \longrightarrow \omega^{*}\left(S, X^{*}\right)
$$

dada por

$$
(\Phi(T)(s))(x)=(T x)(s), \forall s \in S, \forall x \in X,
$$

es un isomorfismo isométrico.

Como estamos especialmente interesados en el conjunto de los operadores que alcanzan la norma y el isomorfismo $\Phi$ nos permite considerar los operadores como funciones $\omega^{*}$-continuas, precisamos de una caracterización de aquéllas funciones que se corresponden con operadores de este tipo.

5.2 Lema ([24, Lemma 2.5.]). Un operador $T \in L(X, C(S))$ alcanza su norma si, y sólo si, existe un punto $s \in S$ tal que

$$
\|\Phi(T)(s)\|=\|T\|
$$

y el funcional $\Phi(T)(s)$ alcanza su norma.

Demostración. Si $T \in N A(X, C(S))$, entonces existe $x \in S_{X}$ de modo que $\|T x\|=\|T\|$. Por tanto,

$$
\|T x\|=|T x(s)|,
$$


para cierto $s \in S$, con lo que

$$
\begin{gathered}
\|\Phi(T)\|=\|T\|=|(T x)(s)|=|\Phi(T)(s)(x)| \leq \\
\leq\|\Phi(T)(s)\| \leq\|\Phi(T)\|,
\end{gathered}
$$

de donde se sigue que

$$
\|T\|=\|\Phi(T)(s)\|=|\Phi(T)(s)(x)|
$$

y $\Phi(T)(s)$ alcanza su norma en $x$.

Recíprocamente; si existe $s \in S$ tal que

$$
\|\Phi(T)(s)\|=\|T\| \text { y }|\Phi(T)(s)(x)|=\|\Phi(T)(s)\|
$$

para cierto vector $x$ de la esfera unidad de $X$, entonces

$$
\begin{aligned}
\|T\| & =\|\Phi(T)(s)\|=|\Phi(T)(s)(x)|= \\
& =|(T x)(s)| \leq\|T x\| \leq\|T\|
\end{aligned}
$$

y $T$ alcanza su norma en $x$.

En el caso particular de ser $X=L_{1}[0,1]$, i.e., el espacio de las funciones definidas en $[0,1]$ que son Lebesgue integrables, tenemos la siguiente caracterización:

5.3 Lema ([35, Proposition 1]). Sea $T \in L\left(L_{1}[0,1], C(S)\right)$. Es condición necesaria y suficiente para que $T$ alcance su norma que existan un punto $s_{0} \in S$ y un subconjunto $E$ de $[0,1]$ medible Lebesgue tales que

$$
\lambda(E)>0
$$

$y$

$$
\left|\psi\left(s_{0}\right)(t)\right|=\sup \{\|\psi(s)\|: s \in S\}, \forall t \in E,
$$


donde $\lambda$ es la medida de Lebesgue y para cada $s \in S, \psi(s)$ es la función de $L_{\infty}[0,1]$ asociada al funcional $\Phi(T)(s)$ en la identificación usual $L_{\infty}[0,1] \equiv\left(L_{1}[0,1]\right)^{*}$.

Demostración. La condición es necesaria: si $T \in N A\left(L_{1}[0,1], C(S)\right)$, el lema anterior nos proporciona un elemento $s_{0} \in S$ tal que

$$
\left\|\Phi(T)\left(s_{0}\right)\right\|=\|T\|
$$

y $\Phi(T)\left(s_{0}\right)$ es un funciunal en $L_{1}[0,1]$ que alcanza su norma. Sea $\psi\left(s_{0}\right)$ el elemento de $L_{\infty}[0,1]$ correspondiente a $\Phi(T)\left(s_{0}\right)$ por el isomorfismo isométrico que identifica a $L_{\infty}[0,1]$ como el dual de $L_{1}[0,1]$. Sea $A \subseteq[0,1]$ un subconjunto medible con $\lambda(A)=0 \mathrm{y}$ de modo que

$$
\left|\psi\left(s_{0}\right)(t)\right| \leq\left\|\psi\left(s_{0}\right)\right\|, \quad \forall t \in[0,1] \backslash A .
$$

Como $\|\psi(s)\|=\|\Phi(T)(s)\| \leq\|T\|$, para cada $s$ de $S$, se tiene de hecho

$$
\left\|\psi\left(s_{0}\right)\right\|=\left\|\Phi(T)\left(s_{0}\right)\right\|=\|T\|=\sup \{\|\psi(s)\|: s \in S\} .
$$

Sea $E$ el conjunto medible Lebesgue dado por

$$
E=\left\{t \in[0,1]:\left|\psi\left(s_{0}\right)(t)\right|=\|T\|\right\} .
$$

Dicho conjunto $E$ ha de verificar $\lambda(E)>0$, ya que, si $\lambda(E)=0$, entonces

$$
\left|\psi\left(s_{0}\right)(t)\right|<\|T\|, \forall t \in[0,1] \backslash(E \cup A),
$$

$\operatorname{con} \lambda(E \cup A)=0$. Como $\Phi(T)\left(s_{0}\right)$ alcanza la norma, existe $f \in S_{L_{1}[0,1]}$ tal que

$$
\left|\int_{0}^{1} \psi\left(s_{0}\right)(t) f(t) d t\right|=\left\|\psi\left(s_{0}\right)\right\|=\|T\|
$$

Pero

$$
\left|\int_{0}^{1} \psi\left(s_{0}\right)(t) f(t) d t\right| \leq \int_{I \backslash(E \cup A)}\left|\psi\left(s_{0}\right)\right||f| d \lambda<
$$




$$
<\int_{I \backslash(E \cup A)}\|T\||f| d \lambda=\|T\|\|f\|_{1}=\|T\|
$$

lo cual es imposible.

Probemos ahora que la condición del enunciado también es suficiente. Supongamos que existen un punto $s_{0}$ de $S$ y un subconjunto $E \subseteq[0,1]$ medible, de medida positiva, tales que

$$
\sup \{\|\psi(s)\|: s \in S\}=\left|\psi\left(s_{0}\right)(t)\right|, \forall t \in E .
$$

Si $\alpha:[0,1] \rightarrow \mathbb{R}$ es una función medible tal que

$$
|\alpha(t)|=1, \forall t \in[0,1]
$$

y

$$
\alpha(t) \psi\left(s_{0}\right)(t)=\left|\psi\left(s_{0}\right)(t)\right|, \forall t \in[0,1],
$$

la función $f:=\frac{\chi_{E}}{\lambda(E)} \alpha\left(\chi_{E}\right.$ es la función característica del conjunto $\left.E\right)$, está en el espacio $L_{1}[0,1]$ y $\|f\|=1$. Además,

$$
\left|\int_{0}^{1} \psi\left(s_{0}\right)(t) f(t) d t\right|=\int_{E} \frac{\left|\psi\left(s_{0}\right)(t)\right|}{\lambda(E)} d t=\left\|\psi\left(s_{0}\right)\right\|
$$

$\mathrm{y}$, por tanto, el funcional $\Phi(T)\left(s_{0}\right)$ alcanza su norma en la función $f$ y

$$
\|\Phi(T)(s)\|=\|\psi(s)\| \leq\left\|\psi\left(s_{0}\right)\right\|=\|T\|
$$

y el resultado anterior nos garantiza que $T$ alcanza la norma.

Dado un espacio topológico $\Omega$ localmente compacto y de Hausdorff, se define $C_{0}(\Omega)$ como el espacio de las funciones continuas $f: \Omega \longrightarrow \mathbb{R}$ que se anulan en el infinito, esto es, para cualquier $\varepsilon>0$, el conjunto

$$
\{\omega \in \Omega:|f(\omega)| \geq \varepsilon\}
$$


es compacto. Considerando en $C_{0}(\Omega)$ la norma uniforme, este espacio es completo. El Teorema de Riesz nor permite identificar el dual de este espacio con el espacio de Banach $\mathcal{M}(\Omega)$ de las medidas de Borel regulares sobre $\Omega$, con la norma dada por

$$
\|\mu\|=|\mu|(\Omega)(\mu \in \mathcal{M}(\Omega)),
$$

siendo $|\mu|$ la variación de la medida real $\mu$.

Los resultados que expondremos ahora nos facilitarán la demostración de la densidad de los operadores que alcanzan la norma entre dos espacios del tipo $C(K)$. Siguiendo en la línea de la Proposición 5.1, trabajaremos con funciones $\omega^{*}$-continuas en lugar de operadores. En el resto de la sección, las letras $K$ y $S$ denotarán dos espacios topológicos compactos y de Hausdorff y $C(K)$ será el espacio de las funciones en $K$ con valores reales.

5.4 Lema. Sea $V$ un subconjunto abierto de K. Entonces, la aplicación de $\mathcal{M}(K)$ en $\mathbf{R}$ definida por

$$
\mu \mapsto|\mu|(V)
$$

es $\omega^{*}$-inferiormente semicontinua.

Demostración. Sea $r>0$ y supongamos que $\mu_{0} \in \mathcal{M}(K)$ verifica

$$
\left|\mu_{0}\right|(V)>r .
$$

Sea $\delta=\left|\mu_{0}\right|(V)-r$; como $\mu_{0 \mid V}$ es una medida de Borel regular en $V$, el Teorema de representación de Riesz nos proporciona un elemento $f \in C_{0}(V)$ tal que $\|f\| \leq 1$ y verifica

$$
\int_{V} f d \mu_{0}>\left|\mu_{0}\right|(V)-\frac{\delta}{2}
$$


Existe entonces una función $h$ en $C(K)$ que extiende a $f$ y de modo que

$$
h(t)=0, \text { si } t \in K \backslash V \text {. }
$$

Si $\mu \in \mathcal{M}(K)$ verifica

$$
\left|\int_{K} h d \mu-\int_{K} h d \mu_{0}\right|<\frac{\delta}{2}
$$

entonces se tiene, usando (1) y (2),

$$
\begin{gathered}
|\mu|(V) \geq\left|\int_{V} h d \mu\right| \geq \\
\geq\left|\int_{V} h d \mu_{0}\right|-\left|\int_{V} h d \mu-\int_{V} h d \mu_{0}\right| \geq \\
\geq\left|\int_{V} f d \mu_{0}\right|-\left|\int_{K} h d \mu-\int_{K} h d \mu_{0}\right|> \\
>\left|\mu_{0}\right|(V)-\delta=r
\end{gathered}
$$

y por tanto, el conjunto $\{\mu \in \mathcal{M}(K):|\mu|(V)>r\}$ es $\omega^{*}$-abierto, para cada $r>0$.

5.5 Lema ([24, Lemma 2.2]). Dados $\mu: S \rightarrow \mathcal{M}(K) \omega^{*}$-continua, $\varepsilon>0, s_{0} \in S$ y $V$ abierto de $K$, existe un entorno abierto $U$ de $s_{0}$ tal que

$$
|\mu(s)|(V)>\left|\mu\left(s_{0}\right)\right|(V)-\varepsilon, \forall s \in U .
$$

Demostración. Es consecuencia inmediata del Lema 5.4 y del hecho de que $\mu$ es $\omega^{*}$-continua.

5.6 Lema ([24, Lemma 2.3]). Sea $\mu: S \longrightarrow \mathcal{M}(K)$ una función $\omega^{*}$-continua y $\delta>0$. Entonces, existen una función $\mu^{\prime}: S \longrightarrow \mathcal{M}(K)$ $\omega^{*}$-continua, un abierto $U$ de $S$, un abierto $V$ de $K$ y una función $h \in C(K)$ tales que 
(i) $\left|\mu^{\prime}(s)\right|(V)=0, \forall s \in U$,

(ii) $\int_{K} h d \mu^{\prime}(s) \geq\left\|\mu^{\prime}\right\|-\delta, \forall s \in U$,

(iii) $\|h\|=1$ y $|h(t)|=1$, si $t \in K \backslash V y$

(iv' $\left\|\mu-\mu^{\prime}\right\| \leq \delta$.

Demostración. La definición de la norma del espacio $\omega^{*}(S, \mathcal{M}(K))$ nos permite elegir un elemento $s_{0} \in S$ verificando

$$
\left\|\mu\left(s_{0}\right)\right\|>\|\mu\|-\delta / 3 \text {. }
$$

Usando el Teorema de descomposición de Hahn y la regularidad de la medida real $\mu\left(s_{0}\right)$, sabemos que existen dos subconjuntos de $S$ cerrados y disjuntos $F^{+}$y $F^{-}$de modo que

$$
\left\|\mu\left(s_{0}\right)\right\| \leq \mu\left(s_{0}\right)^{+}\left(F^{+}\right)+\mu\left(s_{0}\right)^{-}\left(F^{-}\right)+\delta / 3,
$$

siendo $\mu\left(s_{0}\right)^{+}$y $\mu\left(s_{0}\right)^{-}$respectivamente la parte positiva y negativa de la medida real $\mu\left(s_{0}\right)$ que da el Teorema de Hahn. A continuación construimos abiertos disjuntos $V$ y $W$ de $K$ y funciones $h, f \in C(K)$ tales que $h$ verifica (iii) y además

$$
0 \leq f \leq 1, h\left(F^{+}\right)=1=-h\left(F^{-}\right)
$$

y

$$
f_{\mid W}=0, \quad f_{\mid V}=1, \quad F^{+} \cup F^{-} \subseteq W .
$$

Por ser $K$ normal, existe una función $h_{0} \in C(K)$, con

$$
h_{0}\left(F^{+}\right)=1=-h_{0}\left(F^{-}\right) \text {; }
$$

definimos entonces

$$
V=\left\{t \in K:\left|k_{n}(t)\right|<\frac{1}{2}\right\}
$$


y

$$
W=\left\{t \in K: \mid h_{0}(t)>\frac{3}{4}\right\}
$$

Las funciones $f$ y $h$ se pueden conseguir usando de nuevo el Lema de Urysohn. Las condiciones (1), (2) y (3) nos aseguran que

$$
\left|\mu\left(s_{0}\right)\right|(W)>\|\mu\|-\frac{2 \delta}{3}
$$

y, además,

$$
\begin{gathered}
\int_{K} h(1-f) d \mu\left(s_{0}\right) \geq \mu\left(s_{0}\right)\left(F^{+}\right)-\mu\left(s_{0}\right)\left(F^{-}\right)-\frac{\delta}{3} \geq \\
\geq\left\|\mu\left(s_{0}\right)\right\|-\frac{\delta}{3} \geq\|\mu\|-\frac{2 \delta}{3} .
\end{gathered}
$$

De aquí, y en virtud de la $\omega^{*}$-continuidad de $\mu$ y el Lema 5.5 , obtenemos un entorno abierto $U_{0}$ de $s_{0}$ de modo que

$$
\int_{K} h(1-f) d \mu(s)>\|\mu\|-\delta
$$

y

$$
|\mu(s)|(W)>\|\mu\|-\delta
$$

cualquiera que sea $s \in U_{0}$. Sea $U$ un entorno abierto de $s_{0}$ con $\bar{U} \subseteq U_{0}$. Consideremos una función $g \in C(S)$ tal que $0 \leq g \leq 1, g(U)=1$ y $g\left(S \backslash U_{0}\right)=0$. Definimos

$$
\mu^{\prime}: S \longrightarrow \mathcal{M}(K)
$$

por

$$
\mu^{\prime}(s)=(1-g(s) f) \mu(s), \forall s \in S
$$

esto es, $\mu^{\prime}(s)$ es la única medida de Borel en $K$ regular verificando la siguiente igualdad para toda función $\varphi \in C(K)$ :

$$
\int_{K} \varphi d \mu^{\prime}(s)=\int_{K}(1-g(s) f) \varphi d \mu(s) .
$$


Entonces, si $s \in U, g(s)=1$, y como $(1-f)(V)=0$, se verifica (i). Al ser $0 \leq f, g \leq 1$, entonces $\|\mu\| \geq\left\|\mu^{\prime}\right\|$, y este hecho junto con (4) nos permite obtener, para $s \in U$,

$$
\int_{K} h d \mu^{\prime}(s)=\int_{K} h(1-f) d \mu(s)>\|\mu\|-\delta \geq\left\|\mu^{\prime}\right\|-\delta .
$$

Unicamente nos resta comprobar (iv). Si $s \in S \backslash U_{0}$, entonces $g(s)=0$, y por tanto, $\mu(s)=\mu^{\prime}(s)$. Si $s \in U_{0}$, sea $\varphi \in C(K)$ tal que $\|\varphi\| \leq 1$; se tiene, usando (5) y el hecho de que $f_{\mid W}=0$,

$$
\begin{gathered}
\left|\int_{K} \varphi d\left(\mu(s)-\mu^{\prime}(s)\right)\right|=\left|\int_{K} \varphi g(s) f d \mu(s)\right| \leq \\
\leq|\mu(s)|(K \backslash W) \leq\|\mu\|-|\mu(s)|(W)<\delta
\end{gathered}
$$

de donde $\left\|\mu-\mu^{\prime}\right\| \leq \delta$.

5.7 Lema ([24, Lemma 2.4]). Sea $\mu: S \longrightarrow \mathcal{M}(K)$ una función $\omega^{*}$-continua, $\varepsilon>0, U$ un subconjunto abierto de $S, V$ un subconjunto abierto de $K, s_{0} \in U$ y $h \in C(K)$ tales que

(a) $|\mu(s)|(V)=0, \quad \forall s \in U$,

(b) $\int_{K} h d \mu\left(s_{0}\right) \geq\|\mu\|-\varepsilon$,

(c) $\|h\|=1, \quad|h(t)|=1, \quad \forall t \in K \backslash V$.

Entonces, dado $r>2 / 3$, existen $\mu^{\prime}: S \longrightarrow \mathcal{M}(K)$ función $\omega^{*}$-continua $y s_{1}$ elemento de $U$ verificando

(i) $\left|\mu^{\prime}(s)\right|(V)=0, \forall s \in U$,

(ii) $\int_{K} h d \mu^{\prime}\left(s_{1}\right) \geq\left\|\mu^{\prime}\right\|-r \varepsilon$, 
(iii) $\left\|\mu-\mu^{\prime}\right\| \leq r \varepsilon$.

Demostración. Elegimos en primer lugar un número real $\delta>0$ tal que $\delta<\varepsilon(r-2 / 3)$. La $\omega^{*}$-continuidad de $\mu$ y la condición (b) nos proporcionan un entorno abierto $\hat{U}$ de $s_{0}$, que podemos suponer incluido en $U$, de forma que

$$
\int_{K} h d \mu(s)>l i \mu \|-\varepsilon-\delta, \forall s \in \hat{U} .
$$

Sea $M=\sup \{\|\mu(s)\|: s \in \hat{U}\}$. Distinguimos dos casos.

Primer caso. Supongamos que $M \leq\|\mu\|-\varepsilon / 3$.

Elegimos un elemento $a \in K \backslash V$ que ha de cumplir $|h(a)|=1$ y una función $f \in S_{C(S)}$, con $f\left(s_{0}\right)=h(a)$ y $f(S \backslash \hat{U})=0$. Definimos

$$
\mu^{\prime}(s)=\mu(s)+\frac{\varepsilon}{3} f(s) \delta_{a}
$$

donde $\delta_{a}$ es la medida positiva concentrada en el punto $a$, esto es

$$
\delta_{a}(E)=\chi_{E}(a)
$$

para todo subconjunto $E \subseteq K$ Borel-medible. Comprobemos que

$$
\|\mu\| \geq i \mu^{\prime} \|
$$

si $s \in \hat{U}$ y $\varphi \in C(K),\|\varphi\| \leq 1$, es evidente que

$$
\left|\int_{K} \varphi d \mu^{\prime}(s)\right| \leq\|\mu(s)\|+\frac{\varepsilon}{3}\left|\varphi(a)\|f(s) \mid \leq\| \mu(s)\left\|+\frac{\varepsilon}{3} \leq\right\| \mu \|\right.
$$

y si $s \in S \backslash \hat{U}, f(s)=0$ y $\left|\mu^{\prime}(s)\right|=|\mu(s)|$. Es claro que se cumple (i), ya que $|\mu(s)|(V)=0$, para cada $s \in U$, y $\left|\delta_{a}\right|(V)=0$. Como $\left\|\mu^{\prime}\right\| \leq\|\mu\|$, tomando $s_{1}:=s_{0}$, de (b) y (1) se tiene

$$
\int_{K} h d \mu^{\prime}\left(s_{1}\right)=\int_{K} h d \mu\left(s_{1}\right)+\frac{\varepsilon}{3} h(a)^{2}>\|\mu\|-\varepsilon-\delta+\frac{\varepsilon}{3} \geq
$$




$$
\geq\left\|\mu^{\prime}\right\|-\frac{2 \varepsilon}{3}-\delta \geq\left\|\mu^{\prime}\right\|-r \varepsilon,
$$

y se verifica (ii). Nótese que la condición (iii) es inmediata, ya que

$$
\left\|\mu(s)-\mu^{\prime}(s)\right\| \leq \frac{\varepsilon}{3}, \quad \forall s \in S .
$$

Segundo caso. Ahora suponemos que $M>\|\mu\|-\varepsilon / 3$. Elegimos $s_{1} \in \hat{U}$ tal que

$$
\left\|\mu\left(s_{1}\right)\right\|>\|\mu\|-\varepsilon / 3
$$

En primer lugar probaremos que si se dispone de una función $g \in C(K)$ y un abierto $W \subseteq K$ de modo que

$$
\begin{gathered}
0 \leq g \leq 1, \quad g(W)=0, \\
\left|\mu\left(s_{1}\right)\right|(W)>\|\mu\|-\frac{2}{3} \varepsilon-\delta
\end{gathered}
$$

e

$$
\int_{K}(1-g) h d \mu\left(s_{1}\right) \geq\|\mu\|-\frac{2}{3} \varepsilon-\delta
$$

se tiene también la situación descrita en la tesis. En efecto, admitamos que existen semejante función $g \in C(K)$ y un tal abierto $W \subseteq K$ verificando (3), (4) y (5). La condición (4) y el Lema 5.5 nos garantizan la existencia de un entorno abierto $U_{0}$ de $s_{1}$ de forma que si $s \in U_{0}$, entonces

$$
|\mu(s)|(W)>\|\mu\|-\frac{2}{3} \varepsilon-\delta .
$$

Para definir $\mu^{\prime}$ consideremos una nueva función auxiliar $\varphi \in C(S)$ verificando

$$
0 \leq \varphi \leq 1, \varphi\left(s_{1}\right)=1, \text { y } \varphi\left(S \backslash U_{0}\right)=0,
$$

de la que podemos disponer por ser $K$ normal. Entonces, dado $s \in S$, definimos

$$
\mu^{\prime}(s)=(1-\varphi(s) g) \mu(s) .
$$


La condición (i) es inmediata, puesto que $|1-\varphi(s) g| \leq 1$, para cada $s \in S$ y, por tanto, $\left|\mu^{\prime}(s)\right|(E) \leq|\mu(s)|(E)$, para cualquier subconjunto $E \subseteq K$ medible, en particular, $\left\|\mu^{\prime}(s)\right\| \leq\|\mu(s)\|$, para todo $s$ en $S$. Para comprobar (ii) basta notar que

$$
\begin{gathered}
\left\|\mu^{\prime}\right\|-\int_{K} h d \mu^{\prime}\left(s_{1}\right)=\left\|\mu^{\prime}\right\|-\int_{K}(1-g) h d \mu\left(s_{1}\right) \leq \\
\leq\|\mu\|-\int_{K}(1-g) h d \mu\left(s_{1}\right) \leq \frac{2}{3} \varepsilon+\delta \leq r \varepsilon
\end{gathered}
$$

donde se ha usado que $\left\|\mu^{\prime}\right\| \leq\|\mu\|, \varphi\left(s_{1}\right)=1$ y (5). Por último,

$$
\left\|\mu^{\prime}-\mu\right\| \leq r \varepsilon
$$

ya que si $s \in U_{0}$ y $\psi \in B_{C(K)}$, usando las condiciones (3) y (6) se tiene

$$
\begin{gathered}
\left|\int_{K} \psi d \varphi(s) d \mu(s)\right| \leq \int_{K \backslash W}|\psi g \varphi(s)| d|\mu(s)| \leq \\
\leq|\mu(s)|(K \backslash W) \leq \frac{2}{3} \varepsilon+\delta \leq r \varepsilon
\end{gathered}
$$

mientras que si $s \in S \backslash U_{0}, \varphi(s)=0$ y entonces, $\mu(s)=\mu^{\prime}(s)$.

Para finalizar, probemos pues la existencia de una función $g \in C(K)$ y un abierto $W \subseteq K$ verificando (3), (4) y (5). Para ello, consideremos la descomposición de Hahn de la medida $\mu\left(s_{1}\right)$ que nos proporciona una partición de $K$ en dos subconjuntos medibles $E^{+}$y $E^{-}$, donde se concentran la parte positiva y negativa de la medida, respectivamente. Definimos los subconjuntos de $K$ :

$$
P=h^{-1}(\{1\}) \cap E^{+}, N=h^{-1}(\{-1\}) \cap E^{-},
$$

y

$$
Q=h^{-1}(\{1\}) \cap E^{-}, M=h^{-1}(\{-1\}) \cap E^{+}
$$


que evidentemente son medibles. Por ser $|h(t)|=1$, para todo $t \in K \backslash V$, se tiene que $(P \cup N) \backslash V$ y $(Q \cup M) \backslash V$ es una partición de $K \backslash V$. La regularidad de $\mu\left(s_{1}\right)$ y el hecho de que $\left|\mu\left(s_{1}\right)\right|(V)=0$ nos asegura la existencia de dos subconjuntos cerrados, $F_{1}$ y $F_{2}$, tales que

$$
F_{1} \subseteq(P \cup N) \backslash V, \quad F_{2} \subseteq(Q \cup M) \backslash V,
$$

y

$$
\left|\mu\left(s_{1}\right)\right|\left((K \backslash V) \backslash\left(F_{1} \cup F_{2}\right)\right)<\frac{\delta}{6} .
$$

Es clara entonces la igualdad

$$
h(t)\left(1-\chi_{F_{2}}\right)(t)=\left(\chi_{P}-\chi_{N}\right)(t), \forall t \in F_{1} \cup F_{2}
$$

Como $K$ es normal y $F_{1} \cap F_{2}=\emptyset$, existe un abierto $W$ de $K$ tal que $F_{1} \subseteq W$ y $\bar{W} \cap F_{2}=\emptyset$, y, por tanto, una función $g \in C(K)$ con $0 \leq g \leq 1, g\left(F_{2}\right)=1$ y $g(W)=0$, de donde $g$ satisface la condición (3). Se tiene entonces por (8)

$$
h(t)(1-g(t))=\left(\chi_{P}-\chi_{N}\right)(t), \forall t \in F_{1} \cup F_{2},
$$

luego, en virtud de (7),

$$
\begin{gathered}
\int_{K}(1-g) h d \mu\left(s_{1}\right) \geq \int_{K}\left(\chi_{P}-\chi_{N}\right) d \mu\left(s_{1}\right)-\left|\mu\left(s_{1}\right)\right|\left((K \backslash V) \backslash\left(F_{1} \cup F_{2}\right)\right)> \\
>\int_{K}\left(\chi_{P}-\chi_{N}\right) d \mu\left(s_{1}\right)-\delta / 6 \geq\left|\mu\left(s_{1}\right)\right|(P \cup N)-\delta / 6
\end{gathered}
$$

Vamos a probar ahora la desigualdad

$$
\left|\mu\left(s_{1}\right)\right|(P \cup N) \geq\|\mu\|-\frac{2}{3} \varepsilon-\frac{1}{2} \delta
$$

con lo q .e tendremos (5) y además (4), ya que

$$
\left|\mu\left(s_{1}\right)\right|(W) \geq\left|\mu\left(s_{1}\right)\right|\left(F_{1}\right) \geq
$$




$$
\begin{gathered}
\geq\left|\mu\left(s_{1}\right)\right|(P \cup N)-\delta / 6 \geq \\
\geq\|\mu\|-\frac{2}{3} \varepsilon-\delta .
\end{gathered}
$$

Como $\left|\mu\left(s_{1}\right)\right|(V)=0$ y $|h(t)|=1, \forall t \in K \backslash V$,

$$
\int_{K} h d \mu\left(s_{1}\right)=\int_{K}\left(\chi_{h^{-1}(\{1\})}-\chi_{\left.h^{-1}(\{-1\})\right)} d \mu\left(s_{1}\right) .\right.
$$

Entonces,

$$
\begin{gathered}
\left|\mu\left(s_{1}\right)\right|(P \cup N)=\int_{K}\left(\chi_{P}-\chi_{N}\right) d \mu\left(s_{1}\right)= \\
=\int_{K} h d \mu\left(s_{1}\right)-\int_{K}\left(\chi_{Q}-\chi_{M}\right) d \mu\left(s_{1}\right)= \\
=\int_{K} h d \mu\left(s_{1}\right)+\left|\mu\left(s_{1}\right)\right|(Q \cup M)= \\
=\int_{K} h d \mu\left(s_{1}\right)+\left|\mu\left(s_{1}\right)\right|(K \backslash(P \cup N))= \\
=\int_{K} h d \mu\left(s_{1}\right)+\left|\mu\left(s_{1}\right)\right|(K)-\left|\mu\left(s_{1}\right)\right|(P \cup N) \geq \quad \text { (por (1)) } \\
\geq\|\mu\|-\varepsilon-\delta+\left|\mu\left(s_{1}\right)\right|(K)-\left|\mu\left(s_{1}\right)\right|(P \cup N) \geq \quad \text { (por (2)) } \\
\geq 2\|\mu\|-\frac{4}{3} \varepsilon-\delta-\left|\mu\left(s_{1}\right)\right|(P \cup N),
\end{gathered}
$$

$y$, por tanto, tenemos (9).

5.8 Teorema ([24, Theorem 1]). Sean $K$ y $S$ dos espacios topológicos compactos $y$ de Hausdorff. Entonces, el conjunto $N A(C(K), C(S))$ es denso en $L(C(K), C(S))$.

Demostración. Sea $\varepsilon>0$ y $T \in L(C(K), C(S))$, representado en $\omega^{*}\left(S, \mathcal{M}(K)\right.$ ) por la función $\omega^{*}$-continua $\mu: S \longrightarrow \mathcal{M}(K)$ (Proposición 5.1). Tomamos $2 / 3<r<1$ y $\eta>0$ de forma que $\frac{\eta}{1-r}<\varepsilon$. El Lema 
5.6 nos proporciona una aplicación $\mu_{0}: S \rightarrow \mathcal{M}(K) \omega^{*}$-continua, un abierto $U$ en $S$, otro $V$ de $K$ y una función $h \in C(K)$ verificando (i)-(iv). Fijado un elemento $s_{0} \in U_{1}$, el Lema 5.7 garantiza la existencia de una aplicación $\mu_{1}: S \longrightarrow \mathcal{M}(K)$ y $s_{1} \in U$ tales que

$$
\begin{gathered}
\left|\mu_{1}(s)\right|(V)=0, \quad \forall s \in U \\
\left\|\mu_{0}-\mu_{1}\right\| \leq r \eta
\end{gathered}
$$

$$
\int_{K} h d \mu_{1}\left(s_{1}\right) \geq\left\|\mu_{1}\right\|-r \eta .
$$

Por recurrencia, obtenemos una sucesión $\left\{\mu_{n}\right\}_{n \in \mathbb{N}}$ de elementos de $\omega^{*}(S, \mathcal{M}(K))$ y otra de elementos de $U,\left\{s_{n}\right\}_{n \in \mathbb{N}}$, verificando

$$
\left\|\mu_{n-1}-\mu_{n}\right\| \leq r^{n} \eta
$$

$$
\int_{K} h d \mu_{n}\left(s_{n}\right) \geq\left\|\mu_{n}\right\|-r^{n} \eta
$$

Por tanto, de la desigualdad (1) tenemos que la sucesión de operadores $\left\{T_{n}\right\}_{n \in \mathbb{N}}$, siendo $T_{n}$ el operador representado por la función $\mu_{n}$, es de Cauchy, luego converge hacia un operador $\hat{T} \in L(C(K), C(S))$.

Sea $\tau$ la aplicación $\omega^{*}$-continua que representa a $\hat{T}$. Entonces, y haciendo uso de la desigualdad (2),

$$
\begin{gathered}
\hat{T}(h)\left(s_{n}\right)=\int_{K} h d \tau\left(s_{n}\right) \geq \\
\geq \int_{K} h d \mu_{n}\left(s_{n}\right)-\left\|\tau-\mu_{n}\right\| \geq\left\|\mu_{n}\right\|-r^{n} \eta-\left\|\tau-\mu_{n}\right\| .
\end{gathered}
$$

Como

$$
\lim _{n \rightarrow \infty} r^{n} \eta+\left\|\tau-\mu_{n}\right\|=0
$$

tomando límite en la desigualdad anterior, se tiene

$$
\|\hat{T}(h)\| \geq\|\tau\|=\|\hat{T}\| \text {. }
$$


Por tanto, el operador $\hat{T}$ alcanza su norma en $h$ y

$$
\begin{gathered}
\|T-\hat{T}\|=\|\mu-\tau\| \leq\left\|\mu-\mu_{0}\right\|+\sum_{n=1}^{\infty}\left\|\mu_{n}-\mu_{n-1}\right\| \leq \\
\leq \eta+\sum_{n=1}^{\infty} r^{n} \eta=\frac{\eta}{1-r}<\varepsilon
\end{gathered}
$$

y el teorema está probado.

Merece la pena notar que la posible validez del teorema para los espacios de funciones continuas con valores complejos no parece deducirse de forma inmediata de esta demostración. En cualquier caso, los resultados 5.1-5.5 siguen siendo válidos en caso complejo y no es difícil adaptar la demostración del Lema 5.6 a este caso. Sin embargo, el Lema 5.7 no parece tener una extensión inmediata al caso complejo.

Seguidamente, mostramos un ejemplo debido a J. Johnson y J. Wolfe ([25]) de un espacio métrico compacto $S$ con la propiedad de que el conjunto $N A\left(L_{1}[0,1], C(S)\right)$ no es denso en $L\left(L_{1}[0,1], C(S)\right)$. W. Schachermayer probó con anterioridad ([35]), si bien su publicación fue un año posterior, en 1.983 , que el par $\left(L_{1}[0,1], C[0,1]\right)$ también verifica esta propiedad. En cualquier caso, J. Johnson y J. Wolfe se inspiran definitivamente en la construcción de Schachermayer. Sin embargo, coincidimos con las palabras de estos autores cuando afirman que "el ejemplo de Schachermayer es muy extenso e intrincado."

En el resto del apartado, únicamente consideraremos subconjuntos de $[0,1]$ medibles-Lebesgue. En primer lugar, definiremos el espacio $S$. 
Si $S_{0}$ es el subconjunto de $L_{\infty}[0,1] \equiv\left(L_{1}[0,1]\right)^{*}$ formado por las funciones de la forma

$$
\sum_{i=1}^{n}\left(1-2^{-i}\right) \chi_{E_{i}}
$$

donde

$$
n \in \mathbb{N}, E_{i} \subseteq[0,1], \lambda\left(E_{i}\right)<\frac{1}{2^{-i}}
$$

y

$$
E_{i} \cap E_{j}=\emptyset, \text { si } i \neq j, i=1,2, \ldots, n,
$$

entonces el espacio $S$ viene dado por

$$
S=\overline{S_{0}} \omega^{*},
$$

que es un espacio métrico compacto en virtud de la $\omega^{*}$-metrizabilidad de $B_{L_{\infty}[0,1]}([23$, Proposition 27.8]) y el Teorema de Banach-Alouglú.

El resultado anunciado se obtendrá como consecuencia del siguiente lema técnico:

5.9 Lema ([25, Lemma 1]). Sea

$$
s_{0}=\sum_{i=1}^{n}\left(1-2^{-i}\right) \chi E_{i}
$$

un elemento de $S_{0}$ y $\psi: S \rightarrow L_{\infty}[0,1]$ una aplicación $\omega^{*}$-continua tal que para cada $s \in S$,

$$
\|\psi(s)-s\| \leq 1 / 2 \text { y }\|\psi(s)\|_{\infty} \leq 1 .
$$

Entonces,

$$
\left\|\psi\left(s_{0}\right) \chi_{E_{i}}\right\| \leq 1-\frac{1}{2^{-i-2}}, 1 \leq i \leq n
$$


118

Algunos resultados en espacios clásicos

Demostración. Primera parte. Antes de empezar a probar la tesis del lema, hagamos la siguiente observación. Sea

$$
s=\sum_{i=1}^{n} \alpha_{i} \chi_{E_{i}}
$$

con

$$
n \in \mathbb{N}, 0<\alpha_{i}<1, \forall i \text { y } E_{i} \cap E_{j}=\emptyset \text {, si } i \neq j .
$$

Dado $i_{0} \in\{1,2, \ldots, n\}$, fijamos un subconjunto $A$ de $E_{i_{0}} \mathrm{y}$ una partición (medible) $\pi$ de $A$

$$
\pi=\left\{A_{1}, A_{2}, \ldots, A_{k}\right\} \in \Pi(A)
$$

siendo

$$
\Pi(E)=\{\text { particiones finitas (y medibles) de } E\}, \quad(E \subseteq[0,1]) .
$$

Para cada $j \in\{1,2, \ldots, k\}$ sea $C_{j} \subseteq A_{j}$, con

$$
\lambda\left(C_{j}\right)=\frac{2 \alpha_{i_{0}}}{1+\alpha_{i_{0}}} \lambda\left(A_{j}\right)
$$

(véase, por ejemplo, [18, Teorema 2.1.1]) y definimos

$$
s_{\pi}=\frac{1+\alpha_{i_{0}}}{2} \sum_{j=1}^{k} \chi_{C_{j}}+s\left(1-\chi_{A}\right) .
$$

Afirmamos ahora que se verifican las siguientes condiciones:

(i) $\int_{A_{j}} s d \lambda=\int_{A_{j}} \frac{1+\alpha_{i_{0}}}{2} \chi_{C_{j}} d \lambda$.

(ii) $\int_{F} s d \lambda=\int_{F} s_{\pi} d \lambda$, si $F \cap A=\emptyset \circ F$ es unión de una familia de subconjuntos de $\pi$. 
(iii) $\lambda\left(\left\{t \in A: s_{\pi}(t)=0\right\}\right)=\frac{1-\alpha_{i_{0}}}{1+\alpha_{i_{0}}} \lambda(A)$.

(iv) La red $\left\{s_{\pi}\right\}_{\pi \in \Pi(A)}$ converge hacia la función $s$ en la topología $\omega^{*}$, donde $\Pi(A)$ se ordena por refinamiento $\left(\pi_{1} \leq \pi_{2}\right.$ significa que todo elemento de $\pi_{1}$ se expresa como unión finita de elementos de $\pi_{2}$ ).

La afirmación (i) es consecuencia inmediata de la igualdad (1). Para comprobar (ii), notemos que si $F \subseteq[0,1]$ verifica $F \cap A=\emptyset$, entonces $\chi_{C_{j \mid F}}=0$, para cada $j \leq k$ y obtenemos (ii) sin más que usar la definición de $s_{\pi}$; en cambio, si $F$ es unión finita de subconjuntos de $\pi$, (ii) se sigue de (i). Para comprobar (iii) basta notar que los conjuntos $\left\{t \in A: s_{\pi}(t)=\right.$ 0\} y $A \backslash \bigcup_{j=1}^{k} C_{j}$ coinciden, y por tanto

$$
\begin{gathered}
\lambda\left(A \backslash \bigcup_{j=1}^{k} C_{j}\right)=\lambda(A)-\sum_{j=1}^{k} \lambda\left(C_{j}\right)= \\
=\lambda(A)-\sum_{j=1}^{k} \frac{2 \alpha_{i_{0}}}{1+\alpha_{i_{0}}} \lambda\left(A_{j}\right)= \\
=\lambda(A)-\frac{2 \alpha_{i_{0}}}{1+\alpha_{i_{0}}} \lambda(A)=\frac{1-\alpha_{i_{0}}}{1+\alpha_{i_{0}}} \lambda(A)
\end{gathered}
$$

Resta probar (iv), esto es, la red $\left\{s_{\pi}\right\}_{\pi \in \Pi(A)}$ converge a $s$ en la topología $\omega^{*}$ de $L_{\infty}[0,1]\left(\equiv\left(L_{1}[0,1]\right)^{*}\right)$. Para ello elegimos un subconjunto $E \subseteq$ $[0,1]$ y consideramos la partición $\pi_{0} \in \Pi(A)$ dada por

$$
\pi_{0}=\{A \cap E, A \backslash E\}
$$

si $\pi$ es otra partición de $A$ con $\pi \geq \pi_{0}$, entonces, haciendo uso de la condición (ii),

$$
\int_{E} s_{\pi} d \lambda=\int_{E \backslash A} s_{\pi} d \lambda+\int_{E \cap A} s_{\pi} d \lambda=
$$




$$
=\int_{E \backslash A} s d \lambda+\int_{E \cap A} s d \lambda=\int_{E} s d \lambda,
$$

Por tanto, la red $\left\{\int_{E} s_{\pi} d \lambda\right\}_{\pi \in \Pi(A)}$ converge hacia $\int_{E} s d \lambda$. La arbitrariedad del subconjunto $E \subseteq[0,1]$, el hecho de que el subespacio

$$
\operatorname{lin}\left\{\chi_{E}: E \subseteq[0,1]\right\}
$$

es denso en $L_{1}[0,1]$ y la acotación de $\left\{s_{\pi}\right\}_{\pi \in \Pi(A)}$ nos aseguran la convergencia de dicha red a la función $s$ en la topología $\omega^{*}$.

Segunda parte. Supongamos que la tesis del lema es falsa. Por tanto, existe $i_{0} \in\{1,2, \ldots, n\}$, tal que $\left\|\psi\left(s_{0}\right) \chi_{E_{i_{0}}}\right\|>1-2^{-i_{0}-2}$. Como $s_{0} \chi E_{i_{0}}=\left(1-2^{-i_{0}}\right) \chi E_{i_{0}}$ y $\left\|\psi\left(s_{0}\right)-s_{0}\right\| \leq 1 / 2$ por hipótesis, es claro que $\psi\left(s_{0}\right) \chi_{i_{i_{0}}} \geq 0$ y que existe un subconjunto $A \subseteq E_{i_{0}}$, con $\lambda(A)>0$, tal que

y

$$
\int_{A} \psi\left(s_{0}\right) d \lambda>\lambda(A)\left(1-\frac{1}{2^{i_{0}+2}}\right)
$$

$$
\begin{cases}\lambda\left(E_{i_{0}+1}\right)+\lambda(A)<1 / 2^{i_{0}+1}, & \text { si } i_{0}<n \\ \lambda(A)<1 / 2^{i_{0}+1} & \text { si } i_{0}=n .\end{cases}
$$

Si $\pi=\left\{A_{1}, A_{2}, \ldots, A_{k}\right\}$ es una partición de $A$ y $C_{j} \subseteq A_{j}$ verifica (1), para cada $j \leq k$, definimos la función $s_{\pi}$ como en la primera parte; es fácil comprobar que se verifica la igualdad

$$
\begin{gathered}
s_{\pi}=\left(1-\frac{1}{2^{i_{0}+1}}\right) \sum_{j=1}^{k} \chi_{C},+s_{0}\left(1-\chi_{A}\right)= \\
=\left(1-\frac{1}{2^{i_{0}+1}}\right) \sum_{j=1}^{k} \chi_{C_{j}}+\sum_{\substack{j=1 \\
j \neq i_{0}}}^{n}\left(1-2^{-j}\right) \chi_{E}+\left(1-\frac{1}{2^{i_{0}}}\right) \chi_{E_{i_{0}} \backslash A},
\end{gathered}
$$

de donde, en virtud de la condición (3) se deduce que $s_{\pi} \in S_{0} \subseteq S$. Si notamos por

$$
E=\left\{t \in[0,1]: s_{\pi}(t)=0\right\} \cap A,
$$


aplicando la función $\psi$ a $s_{\pi}$, y teniendo en cuenta que por hipótesis $\left\|\psi\left(s_{\pi}\right)\right\| \leq 1$ y $\left\|\psi\left(s_{\pi}\right)-s_{\pi}\right\| \leq 1 / 2$ se obtiene

$$
\begin{aligned}
\int_{A} \psi\left(s_{\pi}\right) d \lambda & =\int_{A \backslash E} \psi\left(s_{\pi}\right) d \lambda+\int_{E} \psi\left(s_{\pi}\right) d \lambda \leq \\
& \leq \lambda(A \backslash E)+\frac{1}{2} \lambda(E) .
\end{aligned}
$$

Como $A \backslash E=\cup_{j=1}^{k} C_{j}$, de la condición (iii) de la primera parte y de (1) obtenemos

$$
\lambda(A \backslash E)+\lambda(E) / 2 \leq \lambda(A)\left(1-\frac{1}{2^{i_{0}+2}}\right)
$$

con lo que la desigualdad anterior nos da

$$
\int_{A} \psi\left(s_{\pi}\right) d \lambda \leq \lambda(A)\left(1-1 / 2^{i_{0}+2}\right)
$$

El apartado iv) garantiza la convergencia de la red $\left\{s_{\pi}\right\}_{\pi \in \Pi(A)}$ a $s_{0}$ y la $\omega^{*}$-continuidad de $\psi$ nos permite obtener

$$
\int_{A} \psi\left(s_{0}\right) d \lambda \leq \lambda(A)\left(1-\frac{1}{2^{i_{0}+2}}\right)
$$

desigualdad que contradice la elección de $A$.

5.10 Teorema ([25, Theorem 1]). Sea $\psi$ un elemento del espacio $\omega^{*}\left(S, L_{\infty}[0,1]\right)$ tal que

$$
\|\psi\| \leq 1 \quad \text { y } \quad\|\psi-i\| \leq 1 / 2
$$

donde $i$ es la inclusión de $S$ en $L_{\infty}[0,1]$. Entonces, se verifica que para cada $s \in S$ y cada natural $k \geq 3$,

$$
\lambda\left(\left\{t \in[0,1]: \psi(s)(t) \geq 1-2^{-k-2}\right\}\right) \leq 2^{-k} 16 .
$$


Demostración. Razonemos por reducción al absurdo. Supongamos que existen $s \in S$ y un natural $k \geq 3$ de modo que se tiene

$$
\lambda\left(\left\{t \in[0,1]: \psi(s)(t) \geq 1-2^{-k-2}\right\}\right)>\frac{16}{2^{k}} .
$$

Podemos entonces encontrar un subconjunto $E \subseteq[0,1]$ con medida $\lambda(E)=16.2^{-k}$ y tal que

$$
\int_{E} \psi(s) d \lambda \geq \lambda(E)\left(1-2^{-k-2}\right)
$$

La $\omega^{*}$-densidad de $S_{0}$ en $S$ y la $\omega^{*}$-continuidad de $\psi$ nos proporcionan para cada $\varepsilon>0$ un elemento $s_{0} \in S_{0}$ tal que

$$
\int_{E} \psi(s) d \lambda \leq \int_{E} \psi\left(s_{0}\right) d \lambda+\varepsilon
$$

Como $s_{0} \in S_{0}, s_{0}$ admite la forma

$$
s_{0}=\sum_{j=1}^{n}\left(1-2^{-j}\right) \chi_{E},
$$

con los subconjuntos $E_{i}$ digjuntos dos a dos. $\mathrm{Si}$

$$
A=\left\{t \in[0,1]: \psi\left(s_{0}\right)(t) \geq 1-2^{-k}\right\}
$$

el Lema 5.9 nos asegura que

$$
\lambda\left(A \cap E_{i}\right)=0, \text { si } i<k-2 .
$$

Además, si $t \in A \backslash \cup_{i=1}^{n} E_{i}$, entonces

$$
\psi\left(s_{0}\right)(t)-s_{0}(t) \geq 1-\frac{1}{2^{k}}>\frac{1}{2}
$$

y como por hipótesis $\left\|\psi\left(s_{0}\right)-s_{0}\right\| \leq 1 / 2$, se tiene que $\lambda\left(A \backslash \cup_{i=1}^{n} E_{i}\right)=0$. Por tanto,

$$
\lambda(A) \leq \sum_{i=k-2}^{n} \lambda\left(E_{i}\right)<\sum_{i=k-2}^{\infty} 2^{-i} \leq \frac{8}{2^{k}}
$$


Como $\lambda(E \cap A) \leq \lambda(A) \leq 8.2^{-k}$ y $\lambda(E)=16 / 2^{k}$, se tiene entonces $\lambda\left(E \cap A^{c}\right) \geq 8 / 2^{k}$ y, por tanto, usando (1) y (2),

$$
\begin{gathered}
\lambda(E)\left(1-\frac{1}{2^{k+2}}\right) \leq \int_{E} \psi(s) d \lambda \leq \int_{E} \psi\left(s_{0}\right) d \lambda+\varepsilon= \\
=\int_{E \cap A} \psi\left(s_{0}\right) d \lambda+\int_{E \cap A^{\varepsilon}} \psi\left(s_{0}\right) d \lambda+\varepsilon \leq \\
\leq \lambda(E \cap A)+\left(1-\frac{1}{2^{k}}\right) \lambda\left(E \cap A^{c}\right)+\varepsilon= \\
=\lambda(E)-\frac{1}{2^{k}} \lambda\left(E \cap A^{c}\right)+\varepsilon \leq \lambda(E)-\frac{8}{2^{2 k}}+\varepsilon= \\
=\lambda(E)\left(1-1 / 2^{k+1}\right)+\varepsilon
\end{gathered}
$$

desigualdad de la que se deduce $1-1 / 2^{k+2} \leq 1-1 / 2^{k+1}$, dada la arbitrariedad de $\varepsilon$, con lo que se tiene una contradicción.

Obtenemos ya el resultado anunciado.

5.11 Corolario ([25, Corollary 2]). Sea $T_{0}: L_{1}[0,1] \longrightarrow C(S)$ el operador dado por

$$
T_{0}(f)(s)=\int_{0}^{1} f(t) s(t) d t, \forall f \in L_{1}[0,1], \forall s \in S
$$

Si $T \in L\left(L_{1}[0,1], C(S)\right)$ es un operador verificando

$$
\|T\|=1 \text { у }\left\|T-T_{0}\right\| \leq 1 / 2,
$$

entonces $T$ no alcanza su norma. En particular, $N A\left(L_{1}[0,1], C(S)\right)$ no es denso en $L\left(L_{1}[0,1], C(S)\right)$. 
Demostración. Sea $T \in L\left(L_{1}[0,1], C(S)\right)$ con $\|T\|=1 \mathrm{y}$ $\left\|T-T_{0}\right\| \leq 1 / 2$ y $\psi$ en $\omega^{*}\left(S, L_{\infty}[0,1]\right)$ la función que a la luz de la Proposición 5.1 representa a $T$. Si $T \in N A\left(L_{1}[0,1], C(S)\right)$, el Lema 5.3 nos proporciona $s \in S$ y $E \subseteq[0,1]$ tales que

$$
\lambda(E)>0 \text { y }|\psi(s)(t)|=1, \forall t \in E .
$$

Puesto que $T_{0}$ es representado por $i$, la inclusión de $S$ en $L_{\infty}[0,1]$, tenemos que

$$
\|\psi-i\| \leq 1 / 2 \text { y }\|\psi\|=1 .
$$

Aplicando el Teorema 5.10, se tiene que

$$
\lambda(\{t \in[0,1]: \psi(s)(t)=1\})=0 .
$$

Por tanto, podemos suponer, sin pérdida de generalidad, que $\psi(s)(t)=-1, \quad \forall t \in E$. Como $\|\psi(s)-s\|_{\infty} \leq 1 / 2$, ha de ocurrir

$$
|\psi(s)(t)-s(t)| \leq 1 / 2, \forall t \in E .
$$

Pero el conjunto

$$
\left\{f \in L_{\infty}[0,1]: \int_{E} f d \lambda \geq 0\right\}
$$

es $\omega^{*}$-cerrado en $I_{\infty}[0,1]$ y además contiene a $S_{0}$, por tanto, contiene a $s \in \bar{S}_{0}{ }^{*}$, lo cual es imposible, puesto que $s(t) \leq-1 / \overline{\mathcal{L}}, \forall t \in E$ y $\lambda(E)>0$.

En general, no ocurre que para cualquier espacio topológico compacto y de Hausdorff $K$, el espacio $C(K)$ tenga la propiedad $A$. De hecho, probaremos que los espacios métricos compactos $K$ para los que el espacio $C(K)$ satisface dicha propiedad $A$, son finitos. 
En primer lugar, establecernos un resultado debido a J. Lindenstrauss que, en caso separable, puede considerarse como una especie de "pseudorecíproco" de la Proposición 3.17. Recordemos el siguiente concepto:

5.12 Definición. Un espacio de Banach $X$ se dice localmente uniformemente convexo (l.u.c.) si para todo $x \in S_{X}$ y toda sucesión $\left\{x_{n}\right\}_{n \in N}$ de puntos de $S_{\boldsymbol{X}}$ tal que

$$
\left\|\frac{x+x_{n}}{2}\right\| \rightarrow 1
$$

se verifica que

$$
\left\|x_{n}-x\right\| \rightarrow 0
$$

5.13 Proposición ([27, Theorem 2.(ii)]). Sea $X$ un espacio de Banach separable que verifica la propiedad A. Entonces, la bola unidad de $X$ es el cierre de la envolvente absolutamente convexa de sus puntos fuertemente expuestos.

Demostración. Per ser $X$ separable, admite una norma |||.|| l.u.c. equivalente a la norma de partida $\|$.$\| (véase [9, Theorem II.2.6]). Pode-$ mos suponer que

$$
\|x\| \leq\|x\|, \quad \forall x \in X .
$$

Tomamos $Y=(X,\|\cdot\| \|) \oplus_{2} \mathbb{K}$, esto es,

$$
\|(x, \lambda)\|=\left(\|x\|^{2}+|\lambda|^{2}\right)^{1 / 2}, \quad \forall x \in X, \lambda \in \mathbb{K}
$$

Resulta inmediato comprobar que el espacio $Y$ dotado de esta norma es l.u.c. Llamamos $C$ al cierre de la envolvente absolutamente convexa de los puntos fuertemente expuestos de $B_{X}$. Si $B_{X} \neq C$, el Teorema del 
bipolar nos proporciona un funcional $x^{*} \in S_{X}$ y un número real $\delta>0$, tales que

$$
\left|x^{*}(x)\right|<1-\delta, \quad \forall x \in C .
$$

Si fijamos $M \geq 2 / \delta$, definimos el operador

$$
T: X \rightarrow Y
$$

por

$$
T x=\left(x, M x^{*}(x)\right) \quad(x \in X) .
$$

Es claro que si componemos $T$ con la proyección sobre el primer factor, obtenemos la identidad en $X$, y se verifica además

$$
\|T\| \geq M \text {. }
$$

Si $x$ es un elemento de $C$ se tiene, por la elección de $x^{*}$ y $M$,

$$
\|T x\| \leq\left(1+M^{2}(1-\delta)^{2}\right)^{1 / 2} \leq M-1 .
$$

Por tanto, si $S \in L(X, Y)$ es tal que $\|S-T\|<1$ y $\|S\|>M$, entonces $S$ no alcanza la norma en un elemento de C, ya que a la luz de (1),

$$
\|S x\|<\|T x\|+1 \leq M, \quad \forall x \in C .
$$

Comc además, la composición de $T$ con la proyección sobre $X$ es un isomorfismo, entonces al verificar $X$ la propiedad $A$, en el conjunto

$$
\{S \in L(X, Y):\|T-S\|<1,\|S\|>M\}
$$

ha de haber algún isomorfismo sobre su imagen $S_{0}$ que alcance la norma en un punto $x_{0} \in B_{X}$, pero como, por ser $Y$ l.u.c. $\frac{S_{0} x_{0}}{\left\|S_{0} x_{0}\right\|}$ es un punto fuertemente expuesto de $B_{Y}$ por cierto funcional $y^{*} \in S_{Y^{*}}$, es inmediato comprobar que $\frac{S_{0}^{*} y^{*}}{\left\|S_{0} x_{0}\right\|}$ expone fuertemente a $x_{0}$, luego $x_{0} \in C$, pero en 
vista de (2) $S_{0}$ no puede alcanzar su norma en un punto de $C$. Por tanto $B_{X}$ debe coincidir con el cierre de la envolvente absolutamente convexa de sus puntos fuertemente expuestos.

5.14 Corolario ([27, Proposition 2.b]). Sea $K$ un espacio métrico compacto. Entonces, el espacio $C(K)$ tiene la propiedad $A$ si, y sólo si, $K$ es finito.

Demostración. Por ser $K$ espacio métrico compacto, el espacio $C(K)$ es separable. Vamos a probar que, si $K$ es infinito, la bola unidad de $C(K)$ carece de puntos fuertemente expuestos. Supongamos que $f \in S_{C(K)}$ es un tal punto; sea pues $\mu \in \mathcal{M}(K)$ la medida que expone fuertemente a $B_{C(K)}$ en $f$. Por tanto,

$$
1=\int_{K} f d \mu
$$

y existe $\delta>0$ tal que

$$
\|f-g\|<1
$$

siempre que

$$
\left|\operatorname{Re} \int_{K}(f-g) d \mu\right|<\delta
$$

Por ser $K$ infinito y $\mu$ regular, existe un abierto $G$ no vacío de forma que $0<|\mu|(G)<\delta / 2$. Usando el Lema de Urysohn es fácil encontrar una función $g \in C(K)$ tal que

$$
\|g\| \leq 1,\|f-g\|=2, \quad \text { y } g(t)=f(t), \quad \forall t \in K \backslash G .
$$

Entonces,

$$
\begin{gathered}
\left|\int_{K}(f-g) d \mu\right|=\left|\int_{G}(f-g) d \mu\right| \leq \\
\leq|\mu|(G)\|f-g\|<\delta
\end{gathered}
$$


por lo que debe ser $\|f-g\|<1$, lo que contradice (1).

Sin embargo, veremos, para finalizar esta sección que para ciertos espacios de Banach $Y$, sí se verifica que $N A(C(K), Y)$ es denso en $L(C(K), Y)$.

5.15 Teorema ([35, Theorem B]). Sea $K$ un espacio topológico compacto $y$ de Hausdorff e $Y$ un espacio de Banach. Entonces, dados $T$ un operador débilmente compacto de $C(K)$ en $Y$ y $\varepsilon>0$, existe un operador $S \in L(C(K), Y)$ que alcanza su norma y de forma que

$$
\|T-S\|<\varepsilon
$$

Antes de dar la demostración de este resultado, obtendremos algunas consecuencias. En primer lugar, notemos que si $Y$ es un espacio de Banach reflexivo, el Teorema de Dieudonné nos asegura que todos los operadores de cualquier espacio Je Banach $X$ en $Y$ son débilmente compactos, obteniendo así:

5.16 Corolario. Si $K$ es un espacio topológico compacto y de Hausdorff eY es un espacio de Banach (real) reflexivo, entonces el conjunto de los operadores que alcanzan la norma de $C(K)$ en $Y$ es denso en el espacio $L(C(K), Y)$. En particular,

$$
\overline{N A\left(C[0,1], l_{2}\right)}=L\left(C[0,1], l_{2}\right)
$$

5.17 Corolario. Si $Y$ es un espacio de Banach que no contiene copia isomórfica alguna de co y $K$ es un espacio topológico compacto $y$ de 
Hausdorff, entonces el conjunto $N A(C(K), Y)$ es denso en el espacio $L(C(K), Y)$. En particular,

$$
\overline{N A\left(C[0,1], L_{1}[0,1]\right)}=L\left(C[0,1], L_{1}[0,1]\right) .
$$

Demostración. En este caso, todo operador es débilmente compacto ([11, Theorem VI.2.15]). Además, $L_{1}$ no contiene copias isomórficas de $c_{0}([17$, Lemma I.3.9]).

Para probar el Teorema 5.15 usaremos una propiedad que caracteriza los subconjuntos débilmente compactos del espacio $\mathcal{M}(K)$, siendo $K$ un espacio topológico compacto y de Hausdorff.

5.18 Definición Sea $\Omega$ un espacio topológico y $W$ un subconjunto del espacio de las medidas de Borel sobre $\Omega$. W se dice uniformemente regular interior si para cada subconjunto (Borel) medible $E$ de $\Omega$ y cada $\varepsilon>0$, existe un subconjunto $K \subseteq E$ compacto tal que

$$
\sup \{|\mu|(E \backslash K): \mu \in W\} \leq \varepsilon .
$$

La propiedad a la cual hacíamos alusión está recogida en el siguiente resultado del que omitimos su demostración:

5.19 Lema ([10, Theorem VII.14]). Sea $K$ un espacio topológico compacto $y$ de Hausdorff y $W$ un subconjunto de $\mathcal{M}(K)$ débilmente compacto. Entonces $W$ es uniformemente regular interior. 
De hecho, este lema es caso particular de un resultado debido a Dieudonné-Grothendieck ([10, Theorem VII.14]) que caracteriza los subconjuntos acotados y relativamente débilmente compactos de $\mathcal{M}(K)$ como aquéllos que son uniformemente regulares interiores.

5.20 Lema ([35, Lemma 3.1]). Sea $K$ un espacio topológico compacto $y$ de Hausdorff $y$ W un subconjunto débilmente compacto del espacio de Banach $\mathcal{M}(K)$. Si $0 \neq \mu_{0} \in \mathcal{M}(K)$ y $\varepsilon>0$, entonces, existen un operador $T_{0} \in L(\mathcal{M}(K))$ con $\left\|T_{0}\right\|=1$ y $f_{0} \in C(K)$ tales que

(i) $\left\|\mu_{0}\right\|=\int_{K} f_{0} d\left(T_{0} \mu_{0}\right), \quad\left\|f_{0}\right\|=1 \quad y$

(ii) $\left\|T_{0} \mu-\mu\right\| \leq \varepsilon, \quad$ si $\mu \in W$.

Demostración. Sea $\mu_{0}=\mu_{0}^{+}-\mu_{0}^{-}$la descomposición de Hahn de la medida real $\mu_{0}$, esto es, existe una partición Borel-medible $\left\{E^{+}, E^{-}\right\}$de $K$, con

$$
\mu_{0}^{+}(A)=\mu_{0}\left(A \cap E^{+}\right), \mu_{0}^{-}(A)=-\mu_{0}\left(A \cap E^{-}\right),
$$

para todo subconjunto $A \subseteq K$ Borel-medible. Dado $\varepsilon>0$, por ser $W$ uniformemente regular interior, existen subconjuntos compactos $K^{+}$y $K^{-}$de $E^{+}$y $E^{-}$respectivamente, de modo que

$$
|\mu|\left(E^{+} \backslash K^{+}\right)+|\mu|\left(E^{-} \backslash K^{-}\right)<\varepsilon / 2, \forall \mu \in W .
$$

Si $x^{+} \in K^{+}$y $x^{-} \in K^{-}$definimos la aplicación

$$
\varphi: K \rightarrow K
$$

de la siguiente forma:

$$
\varphi(x)= \begin{cases}x, & \text { si } x \in K^{+} \cup K^{-} \\ x^{+}, & \text {si } x \in E^{+} \backslash K^{+} \\ x^{-}, & \text {si } x \in E^{-} \backslash K^{-}\end{cases}
$$


Es obvio que $\varphi$ es Borel-medible. A partir de $\varphi$, construimos el operador $T_{0}$ dado por

$$
\begin{gathered}
T_{0}: \mathcal{M}(K) \longrightarrow \mathcal{M}(K) \\
T_{0}(\mu)(f)=\int_{K} f \circ \varphi d \mu, \quad(\mu \in \mathcal{M}(K), \quad f \in C(K)),
\end{gathered}
$$

donde estamos identificando de forma natural $\mathcal{M}(K) \equiv(C(K))^{*} . T_{0}$ es un elemento de $L(\mathcal{M}(K))$, de hecho, $\left\|T_{0}\right\| \leq 1$ ya que si $f \in C(K)$,

$$
\left|T_{0}(\mu)(f)\right| \leq \int_{K}|f \circ \varphi| d|\mu| \leq\|f\|\|\mu\| .
$$

Además, si $\mu \in W$ y $f \in C(K)$, la definición de $\varphi$ y la desigualdad (1) nos permiten obtener

$$
\begin{gathered}
\left|T_{0}(\mu)(f)-\mu(f)\right|=\left|\int_{K}(f \circ \varphi-f) d \mu\right| \leq \\
\leq \int_{K \backslash(K+\cup K-)}|f \circ \varphi-f| d|\mu| \leq 2\|f\||\mu|\left(K \backslash\left(K^{+} \cup K^{-}\right)\right)= \\
=2\|f\|\left(|\mu|\left(E^{+} \backslash K^{+}\right)+|\mu|\left(E^{-} \backslash K^{-}\right)\right) \leq \varepsilon\|f\|,
\end{gathered}
$$

que es la afirmación (ii). Como $K^{+}$y $K^{-}$son compactos disjuntos de $K$, existe una función $f_{0} \in C(K)$ tal que

$$
\left\|f_{0}\right\|=1 \quad \text { y } \quad f_{0 \mid K^{+}}=1=-f_{0 \mid K^{-}}
$$

Como $f_{0} \circ \varphi$ aplica $E^{+}$en 1 y $E^{-}$en -1 , para la función $f_{0}$ se tiene

$$
\begin{aligned}
T_{0}\left(\mu_{0}\right)\left(f_{0}\right)= & \int_{K} f_{0} \circ \varphi d\left(T_{0} \mu_{0}\right)=\int_{K} f_{0} \circ \varphi d \mu_{0}= \\
& =\int_{E^{+}} d \mu_{0}-\int_{E^{-}} d \mu_{0}= \\
& =\mu_{0}\left(E^{+}\right)-\mu_{0}\left(E^{-}\right)=\left\|\mu_{0}\right\|
\end{aligned}
$$

y en particular $\left\|T_{0}\right\|=1$.

El siguiente resultado es de comprobación inmediata. 
5.21 Lema. Sea $X$ e $Y$ espacios de Banach y $T \in L(X, Y)$. Equivalen las siguientes afirmaciones

(i) $T$ es un operador débilmente compacto.

(ii) $T^{* *}$ toma valores en $Y$.

El resultado que presentamos seguidamente, así como su prueba, es caso particular de otro de J. Bourgain ([7, Proposition 1]).

5.22 Lema. Todo subconjunto $K$ débilmente compacto y convexo de un espacio de Banach $X$ tiene la R.N.P.

Demostración. Probemos en primer lugar que $K$ es dentable, esto es,

$$
\forall \varepsilon>0 \exists x \in K: x \notin \overline{c o}\left(K \backslash\left(x+\varepsilon B_{X}\right)\right) \text {. }
$$

En virtud del Lema 4.13 podemos suponer $X$ separable. Consideremos el subconjunto de $K$ débilmente compacto $\overline{\operatorname{Ext}(K)}{ }^{\omega}$, donde $\operatorname{Ext}(K)$ es el conjunto de puntos extremos de $K$, que es un espacio de Baire por ser $w$-compacto. Si $\left\{x_{n}\right\}_{n \in N}$ es una sucesión de elementos de $X$ verificando

$$
X=\longdiv { \operatorname { l i n } \{ x _ { n } : n \in \mathbb { N } \} }
$$

enioncos, en particular,

$$
\overline{\operatorname{Ext}(K)}{ }^{\omega} \subseteq \bigcup_{n=1}^{\infty}\left(x_{n}+\frac{\varepsilon}{4} B_{X}\right)
$$

y como para cada natural $n, x_{n}+\frac{\varepsilon}{4} B_{X}$ es débilmente cerrado, existen $n \in \mathbb{N}$ y $G$ subconjunto de $X$ débilmente abierto tales que

$$
\emptyset \neq \overline{\operatorname{Ext}(K)} \omega^{\omega} \cap G \subseteq x_{n}+\frac{\varepsilon}{4} B_{X} .
$$


Si tomamos

$$
\left.A_{1}=\overline{c o}(G \cap \overline{\operatorname{Exi}(K)})^{\omega}\right)
$$

y

$$
A_{2}=\overline{c o}((K \backslash G) \backslash \overline{\operatorname{Ext}(K)} \omega)
$$

entonces, $A_{1}$ es débilmente compacto $\mathrm{y}$, por $(2)$, $\operatorname{diam}\left(A_{1}\right) \leq \varepsilon / 2$. Pretendemos encontrar un elemento $x \in K$ satisfaciendo (1); para ello elegimos

$$
x \in G \cap \operatorname{Ext}(K)
$$

$x \notin A_{2}$, ya que si $x \in A_{2} \subseteq K$, del Teorema de Krein-Milman se sigue que $x \in K \backslash G$, en contra de la elección de $x$. Definimos ahora un subconjunto auxiliar que nos va a permitir probar (1): si $d=\operatorname{diam}(K)$, sea

$$
B=\left\{(1-t) a_{1}+t a_{2}: a_{1} \in A_{1}, a_{2} \in A_{2}, \frac{\varepsilon}{5 d} \leq t \leq 1\right\},
$$

subconjunto convexo y cerrado de $K$. Comprobemos ahora que $x \notin B$, puesto que si $x \in B$, existen $t \in[\varepsilon / 5 d, 1], a_{1} \in A_{1}, a_{2} \in A_{2}$ tales que

$$
x=(1-t) a_{1}+t a_{2},
$$

y como $x \in \operatorname{Ext}(K)$, entonces $x \in A_{2}$, lo que se ha probado que es imposible. A continuación probamos que

$$
\operatorname{diam}(K \backslash B)<\varepsilon
$$

en efecto, si $x_{1}, x_{2} \in K \backslash B$, existen $a_{1}^{1}, a_{1}^{2} \in A_{1}, a_{2}^{1}, a_{2}^{2} \in A_{2} y$ $t_{1}, t_{2} \in[0, \varepsilon / 5 d[$ tales que

$$
x_{1}=\left(1-t_{1}\right) a_{1}^{1}+t_{1} a_{2}^{1}
$$

y

$$
x_{2}=\left(1-t_{2}\right) a_{1}^{2}+t_{2} a_{2}^{2}
$$

Por tanto,

$$
\left\|x_{1}-x_{2}\right\| \leq\left\|a_{1}^{1}-a_{1}^{2}\right\|+t_{1}\left\|a_{1}^{1}-a_{2}^{1}\right\|+t_{2}\left\|a_{1}^{2}-a_{2}^{2}\right\| \leq
$$




$$
\leq \frac{\varepsilon}{2}+\frac{\varepsilon d}{5 d}+\frac{\varepsilon d}{5 d}=\frac{9}{10} \varepsilon
$$

Así pues, $\operatorname{diam}(K \backslash B)<\varepsilon$ y como sabemos que $x \in K \backslash B$, entonces

$$
K \backslash B \subseteq x+\varepsilon B_{X},
$$

equivalentemente,

$$
K \backslash\left(x+\varepsilon B_{X}\right) \subseteq B
$$

de donde

$$
\overline{c o}\left(K \backslash\left(x+\varepsilon B_{X}\right)\right) \subseteq B
$$

y como $x \notin B$,

$$
x \notin \overline{c \sigma}\left(K \backslash\left(x+\varepsilon B_{X}\right)\right) \text {. }
$$

Si $\emptyset \neq C \subseteq K$ es un subconjunto convexo y cerrado de $K$, entonces es $\omega$-compacto, y, por tanto, es dentable, de donde se deduce que $K$ tiene la R.N.P.

Ya estamos en condiciones de probar el resultado que antes enunciamos

Demostración del Teorema 5.15. Sea $T: C(K) \longrightarrow Y$ un operador débilmente compacto, con $\|T\|=1$. Tomamos

$$
W:=\overline{T^{*}\left(B_{Y} \cdot\right)}
$$

subconjunto de $\mathcal{M}(K)$ débilmente compacto y convexo. El Lema 5.22 nos asegura que $W$ tiene la R.N.P. y en virtud del Corolario 4.12 , dado $\varepsilon>0$, existen $S \in L(\mathcal{M}(K))$ y $\mu_{1} \in W$ tales que

$$
\|S-I\|<\varepsilon / 2
$$

y

$$
\left\|S \mu_{1}\right\|=\max \{\|S \mu\|: \mu \in W\}=\left\|S T^{*}\right\|
$$


El Lema 5.20 aplicado a $\mu_{0}=S\left(\mu_{1}\right) \in \mathcal{M}(K)$ nos da un operador $T_{0} \in L\left(\mathcal{M}(K)\right.$ y una función $f_{0} \in C(K)$, con $\left\|f_{0}\right\|=1=\left\|T_{0}\right\|$ verificando

$$
\left\|T_{0} \mu-\mu\right\| \leq \varepsilon / 2, \forall \mu \in W
$$

esto es, $\left\|T_{0} T^{*}-T^{*}\right\| \leq \varepsilon / 2$ y

$$
\left\|\mu_{0}\right\|=\int_{K} f_{0} d\left(T_{0} \mu_{0}\right)
$$

Definamos $Q:=T_{0} S T^{*}$. Entonces,

$$
\begin{gathered}
\left\|Q-T^{*}\right\| \leq\left\|T_{0} T^{*}-T_{0} S T^{*}\right\|+\left\|T_{0} T^{*}-T^{*}\right\| \leq \\
\leq \varepsilon / 2+\|S-I\|<\varepsilon / 2+\varepsilon / 2=\varepsilon .
\end{gathered}
$$

Veamos que $Q=R^{*}$, para cierto operador $R: C(K) \longrightarrow Y$. Tenemos que $Q^{*}=T^{* *} S^{*} T_{0}^{*}$; además, por ser $T$ débilmente compacto, en virtud del Lema 5.21 se tiene $T^{* *}\left(C(K)^{* *}\right) \subseteq Y$. Por tanto,

$$
Q^{*}: C(K)^{* *} \longrightarrow Y \text {. }
$$

Sea

$$
R=Q_{\mid C(K)}^{*}: C(K) \longrightarrow Y .
$$

Entonces, $Q=R^{*}$,

$$
\|R\|=\|Q\|=\left\|T_{0} S T^{*}\right\| \leq\left\|S T^{*}\right\|
$$

y

$$
\begin{gathered}
\left\|R\left(f_{0}\right)\right\|=\sup \left\{\left|y^{*}\left(R\left(f_{0}\right)\right)\right|: y^{*} \in B_{Y} \cdot\right\}= \\
=\sup \left\{\left|Q y^{*}\left(f_{0}\right)\right|:\left\|y^{*}\right\| \leq 1\right\}= \\
=\sup \left\{\left|T_{0} S \mu\left(f_{0}\right)\right|: \mu \in W\right\} \geq \\
\geq \int_{K} f_{0} d\left(T_{0} \mu_{0}\right)=\left\|S T^{*}\right\|
\end{gathered}
$$


con lo que $R$ alcanza su nurma en $f_{0}$. Además, como $Q=R^{*}$ y $\left\|Q-T^{*}\right\|<\varepsilon$, entonces

$$
\|R-T\|<\varepsilon
$$

Señalemos antes de finalizar esta sección, que A. Iwanik ([21]) estableció la densidad de $N A\left(L_{1}\left(\mu_{1}\right), L_{1}\left(\mu_{2}\right)\right)$, donde $\mu_{1}$ y $\mu_{2}$ son medidas positivas. En particular,

$$
\overline{N A\left(L_{1}[0,1], L_{1}[0,1]\right)}=L\left(L_{1}[0,1], L_{1}[0,1]\right)
$$

\section{B. Los espacios $l_{p}(1<p<\infty)$.}

Continuando con el análisis de las propiedades que verifican los espacios clásicos en relarión con el problema de la densidad de los operadores que alcanzan la norma, nos ocupamos en este apartado de los espacios de sucesiones $l_{p}(1<p<\infty)$. Gracias a los resultados del Capítulo II, sabemos que estos espacios tienen la propiedad $A$. En un trabajo de J. Johnson y J. Wolfe ([24]), publicado en 1.982 , se plantea la cuestión de si también se verifica la propiedad $B$. W. Gowers ([16]) probó en 1990 que existe un espacio de Banach $G$ tal que el conjunto de los operadores de $G$ en $l_{p}(1<p<\infty)$ que alcanzan la norma no es denso en $L\left(G, l_{p}\right)$. La idea que subyace en la demostración de este resultado ya apareció en el contraejemplo debido a Lindenstrauss (Proposición 2.4): $G$ es un espacio cuya bola unidad carece de puntos extremos, $l_{p}$ es estrictamente convexo y existe un operador no compacto (la identidad formal) de $G$ en 
$l_{p}(1<p<\infty)$. En este caso, el mérito de W. Gowers fue el hacer uso del espacio apropiado.

Empezamos por definir el espacio $G$. Para ello, dado $n \in \mathbb{N}$, consideremos la aplicación

$$
\varphi_{n}: l_{\infty} \longrightarrow \mathbb{R}_{0}^{+}
$$

dada por

$$
\varphi_{n}(x)=\frac{1}{H_{n}} \sup \left\{\sum_{j \in J}|x(j)|: J \subseteq \mathbb{N},|J|=n\right\}, \quad\left(x \in l_{\infty}\right),
$$

donde $H_{n}=\sum_{j=1}^{n} 1 / j$ y $|J|$ es el número de elementos del conjunto $J$. Es claro que para cada natural $n, \varphi_{n}$ define en $l_{\infty}$ una norma equivalente a la usual. El espacio $G$ es el subespacio de $l_{\infty}$ formado por las sucesiones $x \in l_{\infty}$ tales que

$$
\left\{\varphi_{n}(x)\right\}_{n \in N} \longrightarrow 0 .
$$

Es inmediato comprobar que la norma dada por

$$
\|x\|=\max \left\{\varphi_{n}(x): n \in \mathbb{N}\right\}, \quad(x \in G),
$$

convierte a $G$ en un espacio de Banach.

Antes de abordar el resultado principal, probemos el siguiente lema, que nos permitirá considerar a $G$ como un subconjunto de $l_{p}$, para todo $1<p<\infty$ :

5.23 Lema ([16]). Dados un número natural $n$ y números reales $\alpha_{1}, \alpha_{2}, \ldots, \alpha_{n} \in \mathbf{R}_{0}^{+}$verificando

$$
\alpha_{1} \geq \alpha_{2} \geq \ldots \geq \alpha_{n}
$$

y

$$
\sum_{j=1}^{k} \alpha_{i} \leq H_{k}, k=1,2, \ldots, n
$$


entonces, para cualquier $1<p<\infty$, se verifica

$$
\sum_{j=1}^{n} \alpha_{j}^{p} \leq \sum_{j=1}^{n}(1 / j)^{p}
$$

Demostración. Para $1<p<\infty$ definimos el subconjunto $K$ de $\mathbb{R}^{n}$ formado por las $n$-uplas de números reales $\left(\alpha_{1}, \alpha_{2}, \ldots, \alpha_{n}\right)$ que verifican

$$
0 \leq \alpha_{n} \leq \alpha_{n-1} \leq \ldots \leq \alpha_{1}, \sum_{j=1}^{k} \alpha_{j} \leq H_{k}, k=1,2, \ldots, n
$$

y la función

$$
\begin{gathered}
\Phi: K \longrightarrow \mathbb{R}_{0}^{+} \text {por } \\
\Phi\left(\alpha_{1}, \alpha_{2}, \ldots, \alpha_{n}\right)=\sum_{j=1}^{n} \alpha_{j}^{p}, \quad\left(\left(\alpha_{1}, \alpha_{2}, \ldots, \alpha_{n}\right) \in K\right) .
\end{gathered}
$$

Probemos que la función continua $\Phi$ definida en el compacto $K$ alcanza su máximo en el punto $(1,1 / 2, \ldots, 1 / n)$. Supongamos que $\Phi$ alcanza el máximo en $\beta=\left(\beta_{1}, \beta_{2}, \ldots, \beta_{n}\right) \neq(1,1 / 2, \ldots, 1 / n)$. Sea entonces

$$
m=\min \left\{r \in\{1,2, \ldots, n\}: \beta_{r}<1 / r\right\} .
$$

Si para cada $k>m$ se verifica

$$
\sum_{j=1}^{k} \beta_{j}<H_{k}
$$

entonces, eligiendo $\eta>0$ de modo que

$$
\sum_{j=1}^{k} \beta_{j}+\eta<H_{k}, \text { para } m \leq k \leq n,
$$

se comprueba inmediatamente que el elemento $\alpha \in \mathbb{R}^{n}$ dado por

$$
\begin{cases}\alpha_{j}=\beta_{j}, & \text { si } j<m \\ \alpha_{m}=\beta_{m}+\eta, & \text { en otro caso }\end{cases}
$$


pertenece a $K$ y es claro que $\Phi\left(\alpha_{1}, \alpha_{2}, \ldots, \alpha_{n}\right)>\Phi\left(\beta_{1}, \beta_{2}, \ldots, \beta_{n}\right)$, lo que contradice la elección de $\left(\beta_{1}, \beta_{2}, \ldots, \beta_{n}\right)$. Sabemos, por tanto, que existe $k>m$ de manera que $\sum_{j=1}^{k} \beta_{j}=H_{k}$; sea entonces

$$
s=\min \left\{k \in\{m+1, m+2, \ldots, n\}: \sum_{j=1}^{k} \beta_{j}=H_{k}\right\} .
$$

Comprobemos que existe $\varepsilon>0$ tal que el elemento $\alpha$ dado por

$$
\left\{\begin{array}{l}
\alpha_{j}=\beta_{j}, \quad \text { si } j \neq m, s \\
\alpha_{m}=\beta_{m}+\varepsilon \\
\alpha_{s}=\beta_{s}-\varepsilon
\end{array}\right.
$$

pertenece a $K$ y de nuevo, se tendrá $\Phi(\alpha)>\Phi(\beta)$, lo cual es imposible. Basta elegir un número real $\varepsilon$ verificando

$$
0<\varepsilon \leq \min \left\{H_{m}-\sum_{j=1}^{m} \beta_{j}, \frac{1}{s}-\frac{1}{s+1}\right\}
$$

y definir $\alpha$ por (1). Es inmediato entonces que

$$
\alpha_{1} \geq \alpha_{2} \geq \ldots \geq \alpha_{n}
$$

si $m=1$ es trivial y si $m>1$, la elección de $m$ nos da $\beta_{j}=1 / j$, si $j<m$, de manera que

$$
\alpha_{m}=\beta_{m}+\varepsilon<1 / m<1 /(m-1)=\alpha_{m-1} .
$$

Como $\beta \in K$, es claro también que

$$
\alpha_{m} \geq \alpha_{m-1} \geq \ldots \geq \alpha_{s} .
$$

Si $s=n$, es obvio que $\alpha \in K$ y si $s<n$, como por la elección de $s$ sabemos que

$$
s \geq 2, \quad \sum_{j=1}^{s-1} \beta_{j}<H_{s-1} \quad \text { y } \quad \sum_{j=1}^{s} \beta_{j}=H_{s}
$$


entonces $\beta_{s}>1 / s ;$ y usando ahora que $\sum_{j=1}^{s+1} \beta_{j} \leq H_{s+1}$, ha de tenerse que $\beta_{s+1} \leq 1 /(s+1)$, de donde

$$
\alpha_{s}=\beta_{s}-\varepsilon>\frac{1}{s}-\varepsilon \geq \frac{1}{s+1} \geq \beta_{s+1}=\alpha_{s+1}
$$

y por tanto también

$$
\alpha_{m} \geq \alpha_{m-1} \geq \ldots \geq \alpha_{s} .
$$

Para terminar basta observar que la función $f:[0, \varepsilon] \longrightarrow \mathbb{R}$ dada por

$$
f(\eta)=\left(\beta_{m}+\eta\right)^{p}+\left(\beta_{s}-\eta\right)^{p}-\beta_{m}^{p}-\beta_{s}^{p}(0<\eta \leq \varepsilon)
$$

tiene derivada positiva en cada punto $0<\eta \leq \varepsilon$, y, por tanto, es estrictamente creciente en $[0, \varepsilon]$ y como $f(0)=0$, entonces $f(\varepsilon)>0$, pero

$$
f(\varepsilon)=\sum_{j=1}^{n} \alpha_{j}^{p}-\sum_{j=1}^{n} \beta_{j}^{p}
$$

lo que es absurdo, ya que $\Phi$ alcanza su máximo en $\beta$.

Nótese que $G$ es subconjunto de co ya que si $x$ es una sucesión de escalares que no converge a cero, existe $\varepsilon>0$ tal que el conjunto

$$
I=\{j \in \mathbb{N}:|x(j)| \geq \varepsilon\}
$$

es infinito. Así, dado $n \in \mathbb{N}$, existe $J_{n} \subseteq I$ de modo que $\left|J_{n}\right|=n$, obteniéndose

$$
\varphi_{n}(x) \geq \frac{1}{H_{n}} \sum_{j \in J_{n}}|x(j)| \geq \frac{n}{H_{n}} \varepsilon
$$

y como consecuencia, la sucesión $\left\{\phi_{n}(x)\right\}_{n \in \mathbb{N}}$ no converge a cero y $x \notin G$. De aquí es fácil comprobar que para todo $x \in G$ y todo $n \in \mathbb{N}$ el supremo que define a $\varphi_{n}(x)$ se alcanza, puesto que como en particular $x \in c_{0}$, existen $j_{1}, j_{2}, \ldots, j_{n} \in \mathbb{N}$ verificando

$$
\left|x\left(j_{1}\right)\right| \geq\left|x\left(j_{2}\right)\right| \geq \ldots \geq\left|x\left(j_{n}\right)\right| \geq|x(k)|, \forall k \neq j_{1}, j_{2}, \ldots, j_{n},
$$


de donde

$$
\sup \left\{\sum_{j \in J}|x(j)|:|J|=n, J \subseteq \mathbb{N}\right\}=\left|x\left(j_{1}\right)\right|+\left|x\left(j_{2}\right)\right|+\ldots+\left|x\left(j_{n}\right)\right|
$$

G.

El siguiente resultado recoge algunas de las propiedades del espacio

5.24 Teorema ([16] [2, Lemma 1.3]).

(i) La sucesión $\left\{e_{n}\right\}_{n \in \mathrm{N}}$ es una base de $G$, siendo $e_{n}(m)=0$, si $n \neq m, e_{n}(n)=1$.

(ii) La bola unidad de $G$ no tiene puntos extremos. Más aún, dado $x \in S_{G}$, existen $\delta>0$ y $\nu \in \mathbb{N}$ tales que

$$
\left\|x+\lambda e_{n}\right\| \leq 1, \text { para } n \geq \nu \text { y }|\lambda| \leq \delta .
$$

(iii) $G$ es subespacio de $l_{p}(1<p<\infty)$ y la inclusión de $G$ en $l_{p}$ es un operador no compacto.

Demostración. (i) Vamos a probar que cada elemento $x \in G$ se expresa de la forma (evidentemente única)

$$
x=\sum_{n=1}^{\infty} x(n) e_{n}
$$

Basta comprobar que la sucesión de sumas parciales es de Cauchy en $(G,\|\|$.$) con lo que será convergente en G$ y, por tanto, en $c_{0}$, luego la suma deberá ser $x$. Dado $\varepsilon>0$, por la definición de $G$, existe $\nu_{0} \in \mathbb{N}$ tal 
que si $n \geq \nu_{0}$, entonces $\varphi_{n}(x)<\varepsilon$, y por ser $x$ convergente a cero, existe otro nat:ural $\nu$ tal que

$$
|x(n)|<\varepsilon / \nu_{0}, \text { si } n \geq \nu .
$$

Veamos que si $n \geq \nu$ y $h \in \mathbb{N}$, entonces

$$
\left\|\sum_{j=n+1}^{n+h} x(j) e_{j}\right\|<\varepsilon .
$$

Para un natural $k$ posterior a $\nu_{0}$ tenemos

$$
\varphi_{k}\left(\sum_{j=n+1}^{n+h} x(j) e_{j}\right) \leq \varphi_{k}(x)<\varepsilon
$$

mientras que si $k<\nu_{0}$, y puesto que $n \geq \nu_{0}$,

$$
\varphi_{k}\left(\sum_{j=n+1}^{n+h} x(j) e_{j}\right)<\frac{1}{H_{k}} \frac{\varepsilon}{\nu_{0}} k \leq \frac{\varepsilon k}{\nu_{0}}<\varepsilon
$$

(ii) Sea $x \in G$, con $\|x\|=1$. Nuevamente podemos encontrar un natural $\nu_{n}$ de forma que

$$
\varphi_{n}(x)<1 / 2, \quad \forall n \geq \nu_{0},
$$

$\mathrm{y}$ otro natural $\nu_{1}$ tal que

$$
|x(n)|<1 / 2 \nu_{0}, \text { si } n \geq \nu_{1} .
$$

Fijamos $0<\delta<1 / 2 \nu_{0}$. Si $|\lambda| \leq \delta$ y $n \geq \nu_{1}$, entonces probaremos que

$$
\left\|x+\lambda e_{n}\right\| \leq 1 \text {. }
$$

En efecto; si $k$ es un natural posterior a $\nu_{0}$, entonces

$$
\varphi_{k}\left(x+\lambda e_{n}\right) \leq \varphi_{k}(x)+\delta<1 / 2+\delta<1
$$


y si en cambio $k \leq \nu_{0}$, dado $J \subseteq \mathbb{N}$ con $|J|=k$, en caso en que $n \notin J$,

$$
\sum_{j \in J}\left|x(j)+\lambda e_{n}(j)\right|=\sum_{j \in J}|x(j)| \leq H_{k},
$$

mientras que si $n \in J$,

$$
\begin{gathered}
\sum_{j \in J}\left|x(j)+\lambda e_{n}(j)\right|= \\
=|x(n)+\lambda|+\sum_{j \in J \backslash\{n\}}|x(j)| \leq|x(n)|+\delta+\sum_{j \in J \backslash\{n\}}|x(j)| \leq \\
\leq \frac{1}{2 \nu_{0}}+\frac{1}{2 \nu_{0}}+H_{k-1}=\frac{1}{\nu_{0}}+H_{k-1} \leq H_{k} .
\end{gathered}
$$

Por tanto, $\varphi_{k}(x) \leq 1$, para todo $k$, luego $\left\|x+\lambda e_{n}\right\| \leq 1$.

(iii) Sea $1<p<\infty$ y $x$ un elemento de $G$ que podemos suponer en la bola unidad. Si $n$ es natural, entonces, reordenando la $n$-upla $(|x(1)|,|x(2)|, \ldots,|x(n)|)$ decrecientemente y usandc que

$$
\sum_{j=1}^{k}|x(j)| \leq H_{k}, k=1,2, \ldots, n,
$$

el lema anterior nos asegura que

$$
\sum_{j=1}^{n}|x(j)|^{p} \leq \sum_{j=1}^{\infty}(1 / j)^{p}
$$

lo que dice que $x \in l_{p}$ y, por tanto, la inclusión de $G$ en $l_{p}$ es un operador; es claro que no es un operador compacto, ya que la sucesión $\left\{e_{n}\right\}_{n \in N}$ en $l_{p}$ no es un conjunto relativamente compacto.

Para el apartado (ii), W. Gowers únicamente prueba que dado $x \in S_{G}$, existen $\delta>0$ y $\nu$ tales que $\left\|x \pm \delta e_{\nu}\right\|=1$. Este hecho, junto con el 
apartado (iii), ya le permite deducir la no densidad de $N A\left(G, l_{p}\right)$ en $L\left(G, l_{p}\right)(1<p<\infty)$.

No obstante, el apartado (ii) tal como ha sido presentado (véase [2]) permite establecer dos resultados el primero de los cuales es análogo a la Proposición 2.4, y el segundo, formalmente más general que la no densidad de los operadores que alcanzan la norma de $G$ en $l_{p}$.

5.25 Corolario ([2, Theorem 1.4]). Sea $Y$ un espacio de Banach estrictamente convexo y $T \in N A(G, Y)$. Entonces, existe $\nu \in \mathbb{N}$ tal que

$$
T e_{n}=0, \text { si } n \geq \nu \text {. }
$$

Por tanto, $T$ es un operador de rango finito y si hay un operador no com pacto de $G$ en $Y$, entonces $N A(G, Y)$ no es denso en el correspondiente espacio de operadores.

Demostración. Sea $T \in L(G, Y)$ y $x \in S_{G}$ verificando $\|T x\|=\|T\|$. Por la afirmación (ii) del Teorema 5.24 , existen $\delta>0$ y $\nu \in \mathbb{N}$ tales que

$$
\left\|x+\lambda e_{n}\right\| \leq 1, \text { si }|\lambda| \leq \delta \text { y } n \geq \nu .
$$

Para un natural $n$ posterior a $\nu$ tenemos

$$
T x=\frac{1}{2}\left(T x+\delta T e_{n}\right)+\frac{1}{2}\left(T x-\delta T e_{n}\right) .
$$

Por tanto, $T e_{n}=0$, si $n \geq \nu$, puesto que $Y$ es estrictamente convexo.

Particularizando el resultado anterior al caso $Y=l_{p}$, como la identidad formal de $G$ en $l_{p}$ es un operador no compacto, (apartado (iii) del Teorema 5.24) obtenemos 
5.26 Corolario ([3, Corollary 1.5]). Ningún espacio de Banach estrictamente convexo que contenga a $l_{p}$ isomórficamente $(1<p<\infty)$ tiene la propiedad $B$. Por tanto, $L_{p}[0,1](1<p<\infty)$ no tiene la propiedad $B$.

De hecho, si $\mu$ es una medida positiva y $L_{p}(\mu) \supseteq l_{p}(1<p<\infty)$, entonces tampoco tiene la propiedad $B$. Para el caso $p=1$, sabemos que $L_{1}[0,1]$ no tiene la propiedad $A$ (Corolario 5.11). Aún está abierto el problema que W. Gowers resolvió para los espacios $l_{p}(1<p<\infty)$ para $p=1$ :

5.27 Problema. ¿Tiene $l_{1}$ la propiedad B?

\section{C. El problema de Bishop-Phelps para la clase de los operadores compactos.}

Presentamos finalmente un resultado sobre el Problema de BishopPhelps para la clase de los operadores compactos. Dados $X$ e $Y$ espacios de Banach, notaremos por $K(X, Y)$ (resp. $F R(X, Y)$ ) el espacio de los operadores compactos (resp. de rango finito) de $X$ en $Y$ y por $K N A(X, Y)$ (resp. FRNA $(X, Y)$ ) el conjunto de los operadores compactos (resp. de rango finito) de $X$ en $Y$ que alcanzan la norma. El objetivo de este apartado es probar los dos siguientes teoremas ([24]): 
5.28 Teorema. Si $X=C(K)$, el espacio de las funciones (reales o complejas) aefinidas en el espacio topológico $K$ compacto $y$ de Hausdorff o $X=L_{1}(\mu)$, siendo $\mu$ una medida positiva $y$ finita, entonces el conjunto FRNA $(X, Y)$ es denso en el espacio $K(X, Y)$, cualquiera que sea $Y$ espacio de Barach. En particular, $\overline{K N A(X, Y)}=K(X, Y)$.

5.29 Teorema. Para todo espacio de Banach $X$, el conjunto FRN $A(X, Y)$ es denso en $K(X, Y)$, donde $Y$ es un espacio del tipo $C(K)$ o el espacio de funciones reales $L_{1}(\mu) y$, por tanto, $\overline{K N A(X, Y)}=K(X, Y)$.

Observemos que, a diferencia de lo contemplado hasta ahora en este capítulo, se considera también el espacio de funciones continuas con valores complejos.

Para la demostración del primero de estos resultados seguiremos el siguiente esquema: dado un operador compacto $T: X \rightarrow Y$ comprobaremos que se puede aproximar en norma por un operador $T_{0}: X \rightarrow Y$ de rango finito, y que para $X=C(K) \circ L_{1}(\mu)$, se verifica

$$
\overline{F R N A(X, Y)}=F R(X, Y) \text {. }
$$

Introducimos seguidaiıente una propiedad que nos permitirá probar, para los espacios $X$ que se consideran, la densidad de $F R(X, Y)$ en $K(X, Y)$.

5.30 Definición. Un espacio de Banach $Y$ satisface la propiedad de aproximación (P.A. en adelante) si dados $\varepsilon>0$ y $K$ subconjunto compacto de $Y$, existe un elemento $T$ de $F R(Y)$ tal que

$$
\|T y-y\| \leq \varepsilon, \forall y \in K \text {. }
$$


Es fácil comprobar que todo espacio de Banach con una base de Schauder tiene la P.A.

Observemos además que si $Y$ es un espacio de Banach satisfaciendo la P.A., entonces el conjunto $F R(X, Y)$ es denso en el espacio $K(X, Y)$, para todo espacio de Banach $X$.

En efecto, si $X$ es un espacio de Banach, $T \in K(X, Y)$ y $\varepsilon>0$, basta tomar $K:=\overline{T\left(B_{X}\right)}$ y la P.A. nos proporciona un operador $T_{0}$ de rango finito, tal que

$$
\left\|T_{0} y-y\right\| \leq \varepsilon, \forall y \in K
$$

En particular,

$$
\left\|T_{0} T-T\right\| \leq \varepsilon
$$

Probemos inicialmente que los espacios $C(K)$ y $L_{1}(\mu)$ verifican la P.A., para lo cual usaremos el siguiente resuitado:

5.31 Lema. Sea $Y$ un espacio de Banach y $\left\{P_{\lambda}\right\}_{\lambda \in \Lambda}$ una red acotada de operadores de rango finito en $Y$ tal que

$$
\left\{\left\|P_{\lambda} y-y\right\|\right\}_{\lambda \in \Lambda} \longrightarrow 0, \forall y \in Y .
$$

Entonces $Y$ tiene la propiedad de aproximación.

Demostración. Por hipótesis, la red $\left\{P_{\lambda}\right\}_{\lambda \in \Lambda}$ converge puntualmente al operador identidad y es acotada en norma. Un argumento estándar permite comprobar que converge uniformemente a I sobre conjuntos compactos.

5.32 Proposición. (i) Si $\mu$ es una medida positiva, entonces el espacio $L_{1}(\mu)$ tiene la P.A. 
(ii) Si $K$ es un espacio topológico compacto $y$ de Hausdorff, entonces $C(K)$ tiene la P.A.

Demostración. (i) Sea $\mu$ una medida positiva en un conjunto $\Omega$ y consideremos el conjunto $\Lambda$ formado por elementos del tipo

$$
\begin{gathered}
\lambda=\left(A_{1}, A_{2}, \ldots, A_{n}\right): n \in \mathbb{N}, A_{i} \subseteq \Omega, A_{i} \text { es medible, } \\
0<\mu\left(A_{i}\right)<\infty, 1 \leq i \leq n, \quad A_{i} \cap A_{j}=\emptyset, \quad i \neq j,
\end{gathered}
$$

ordenado por refinamiento: $\left(A_{1}, A_{2}, \ldots, A_{n}\right) \leq\left(B_{1}, B_{2}, \ldots, B_{m}\right)$ quiere decir que todo conjunto $A_{i}$ se expresa como unión finita de conjuntos de $\left(B_{1}, B_{2}, \ldots, B_{m}\right) \cdot(\Lambda, \leq)$ es un conjunto dirigido.

Dado $\lambda=\left(A_{1}, A_{2}, \ldots, A_{n}\right) \in \Lambda$, definimos

$$
\begin{gathered}
P_{\lambda}: L_{1}(\mu) \rightarrow L_{1}(\mu) \text { por } \\
P_{\lambda}(f)=\sum_{i=1}^{n}\left(\frac{1}{\mu\left(A_{i}\right)} \int_{A_{i}} f d \mu\right) \chi_{A_{i}}, \quad\left(f \in L_{1}(\mu)\right) .
\end{gathered}
$$

El operador $P_{\lambda}$ tiene rango finito y se comprueba sin dificultad que $\left\|P_{\lambda}\right\|=1$. Además, probaremos que

$$
\left\{P_{\lambda}(f)\right\}_{\lambda \in \Lambda} \longrightarrow f, \forall f \in L_{1}(\mu) .
$$

Si $f=\chi_{A}$, con $A$ medible y $0<\mu(A)<\infty$ y $\lambda \geq \lambda_{0}=(A)$, entonces $P_{\lambda}\left(\chi_{A}\right)=\chi_{A}$. Por tanto, si $f \in \operatorname{lin}\left\{\chi_{E}: 0<\mu(E)<\infty\right\}, P_{\lambda}(f)=f$ a partir de cierto $\lambda_{0} \in \Lambda$. Como el conjunto

$$
\left\{f \in L_{1}(\mu): P_{\lambda}(f) \rightarrow f\right\}
$$

es un subespacio de $L_{1}(\mu)$ que es cerrado por el hecho de ser la familia $\left\{P_{\lambda}: \lambda \in \Lambda\right\}$ uniformemente acotada, y hemos comprobado que dicho subespacio contiene al subespacio

$$
\operatorname{lin}\left\{\chi_{E}: 0<\mu(E)<\infty\right\}
$$


entonces, coincide con $L_{1}(\mu)$.

(ii) Sea $\Lambda$ el conjunto de todos los recubrimientos finitos por abiertos (no vacios) de $K$ y ordenados de la forma siguiente:

$$
\left(U_{1}, U_{2}, \ldots, U_{n}\right) \leq\left(V_{1}, V_{2}, \ldots, V_{m}\right)
$$

cuando, y sólo cuando,

$$
\forall j \in\{1,2, \ldots, m\} \exists i \in\{1,2, \ldots, n\} \text { tal que } V_{j} \subseteq U_{i} .
$$

Obviamente, el par $(\Lambda, \leq)$ es dirigido. Sea $\lambda=\left(U_{1}, U_{2}, \ldots, U_{n}\right)$ un elemento de $\Lambda$. Si elegimos una partición de la unidad $\left\{\varphi_{1}, \varphi_{2}, \ldots, \varphi_{n}\right\}$ asociada al recubrimiento $\lambda$ de $K$ y para cada $i \in\{1,2, \ldots, n\}$ un punto $t_{i} \in U_{i}$, definimos entonces

$$
\begin{gathered}
P_{\lambda}: C(K) \rightarrow C(K) \text { por } \\
P_{\lambda}(f)=\sum_{i=1}^{n} f\left(t_{i}\right) \varphi_{i}, \quad(f \in C(K)) .
\end{gathered}
$$

Claramente $\left\|P_{\lambda}\right\|=1$ y $P_{\lambda} \in F R(C(K))$. Para finalizar, probemos que la red $\left\{P_{\lambda}\right\}_{\lambda \in \Lambda}$ converge puntualmente al operador identidad. Dados $f \in C(K)$ y $\varepsilon>0$, la compacidad de $K$ nos proporciona un elemento $\lambda_{0}=\left(U_{1}, U_{2}, \ldots, U_{n}\right)$ de $\Lambda$ de modo que si $i \leq n$ y $s, t \in U_{i}$, tenemos

$$
|f(t)-f(s)|<\varepsilon \text {. }
$$

Si $\lambda=\left(V_{1}, V_{2}, \ldots, V_{m}\right)$ es un elemento de $\Lambda$, con $\lambda \geq \lambda_{0}$, y $t$ es un elemento de $K$, consideramos el conjunto $F_{t}:=\left\{i \in\{1,2, \ldots, m\}: t \in V_{i}\right\}$, y es claro que

$$
\begin{gathered}
\left|P_{\lambda}(f)(t)-f(t)\right|=\left|\sum_{i=1}^{m} f\left(t_{i}\right) \varphi_{i}(t)-f(t)\right|= \\
=\left|\sum_{i=1}^{m}\left(f\left(t_{i}\right)-f(t)\right) \varphi_{i}(t)\right| \leq \sum_{i \in F_{i}}\left|f\left(t_{i}\right)-f(t)\right|\left|\varphi_{i}(t)\right|<\varepsilon,
\end{gathered}
$$


luego

$$
\left\|P_{\lambda}(f)-f\right\| \leq \varepsilon, \text { si } \lambda \geq \lambda_{0}
$$

Ya sabemos que si un espacio de Banach $Y$ tiene la P.A. entonces $F R(X, Y)$ es denso en $K(X, Y)$, para cualquier espacio de Banach $X$. Como en nuestro caso necesitamos que $\overline{F R(X, Y)}=K(X, Y)$, para $X=C(K)$ o $L_{1}(\mu)$ e $Y$ un espacio de Banach arbitrario, daremos una condición sobre $X$ ( $X^{*}$ tiene la P.A.) suficiente para que ocurra ésto. El siguiente lema técnico, caso particular del Principio de reflexividad local, nos será de utilidad (omitimos la demostración).

5.33 Lema ([28, Lemma 1.e.6]). Dados $Y$ un espacio de Banach, $F$ un subespacio finito dimensional de $Y^{* *} y \varepsilon>0$, existe un operador $T_{0}: F \longrightarrow Y$ tal que $\left\|T_{0}\right\| \leq 1+\varepsilon y T_{0 \mid F n Y}$ es la inclusión natural de $F \cap Y$ en $Y$.

5.34 Proposición ([28, Theorem 1.e.5]). Sea $X$ un espacio de Banach tal que $X^{*}$ verifica la P.A. Entonces, para todo espacio de Banach $Y$, todo operador compacto $T: X \longrightarrow Y$ y tedo $\varepsilon>0$, existe $T_{0} \in F R(X, Y)$ tal que $\left\|T-T_{0}\right\|<\varepsilon$.

Demostración. Supongamos que $X^{*}$ verifca la P.A. Si $Y$ es un espacio de Banach, $T \in K(X, Y)$ y $0<\varepsilon<1 / 2$, entonces, como existen subconjuntos $\left\{y_{1}^{* *}, y_{2}^{* *}, \ldots, y_{n}^{* *}\right\}$ de $Y^{* *}$ y $\left\{x_{1}^{*}, x_{2}^{*}, \ldots, x_{n}^{*}\right\}$ de $X^{*}$ de modo que

$$
\left\|T^{*}-\sum_{i=1}^{n} y_{i}^{* *} \otimes x_{i}^{*}\right\| \leq \varepsilon / 4
$$


en particular, si $x \in B_{X}$,

$$
\left\|T x-\sum_{i=1}^{n} x_{i}^{*}(x) y_{i}^{* *}\right\| \leq \varepsilon / 4
$$

Si los elementos $y_{i}^{* *}$ estuviesen en $Y$, nuetra demostración habría concluido. El lema anterior resuelve esta situación, cambiando cada elemento $y_{i}^{* *}$ de $Y^{* *}$ por otro de $Y$. La compacidad de $T$ garantiza la existencia de cierto subconjunto $\left\{x_{1}, x_{2}, \ldots, x_{m}\right\}$ de $X$ verificando

$$
T B_{X} \subseteq \bigcup_{j=1}^{m} T x_{j}+\frac{\varepsilon}{4} B_{Y} .
$$

Aplicamos el lema anterior al subespacio

$$
F:=\operatorname{lin}\left\{T x_{1}, T x_{2}, \ldots, T x_{m}, y_{1}^{* *}, y_{2}^{* *}, \ldots, y_{n}^{* *}\right\} \subseteq Y^{* *}
$$

Por tanto, existe un operador $T_{0} \in L(F, Y)$ tal que $\left\|T_{0}\right\| \leq 1+\varepsilon$ y $T_{0 \mid F \cap Y}$ es la inclusión de $F \cap Y$ en $Y$. En virtud de (2), si $x \in B_{X}$, existe $j \leq m$ tal que

$$
\left\|T x-T x_{j}\right\| \leq \varepsilon / 4
$$

así, sin más que aplicar las propiedades de $T_{0}$, obtenemos

$$
\begin{gathered}
\left\|T x-\sum_{i=1}^{n} x_{i}^{*}(x) T_{0}\left(y_{i}^{* *}\right)\right\| \leq \\
\leq\left\|T x-T x_{j}\right\|+\left\|T x_{j}-\sum_{i=1}^{n} x_{i}^{*}(x) T_{0}\left(y_{i}^{* *}\right)\right\| \leq \\
\leq \frac{\varepsilon}{4}+\left\|T_{0}\left(T x_{j}-\sum_{i=1}^{n} x_{i}^{*}(x) y_{i}^{* *}\right)\right\| \leq \\
\leq \frac{\varepsilon}{4}+(1+\varepsilon)\left\|T x_{j}-\sum_{i=1}^{n} x_{i}^{*}(x) y_{i}^{* *}\right\| \leq
\end{gathered}
$$




$$
\begin{gathered}
\leq \frac{\varepsilon}{4}+(1+\varepsilon)\left(\left\|T x_{j}-T x\right\|+\left\|T x-\sum_{i=1}^{n} x_{i}^{*}(x) y_{i}^{* *}\right\|\right) \leq(\text { por }(1)) \\
\leq \frac{\varepsilon}{4}+(1+\varepsilon)\left(-\frac{\varepsilon}{4}+\frac{\varepsilon}{4}\right) \leq \varepsilon
\end{gathered}
$$

Como ya hemos señalado, vamos a probar que cuando $X=C(K)$ o $L_{1}(\mu)$, se verifica $\overline{F R N A(X, Y)}=F R(X, Y)$, para cualquier espacio de Banach $Y$; la clave se encuentra en el siguiente enunciado:

5.35 Proposición ([24, Lemma 3.1]). Sea $X$ un espacio de Banach. Las dos siguientes afirmaciones equivalen:

(i) Dados un espacio normado $F$ finito dimensional, $T \in L(X, F)$ y $\varepsilon>0$, existe $P: X \rightarrow X$ proyección de rango finito y norma uno tal que

$$
\|T-T P\| \leq \varepsilon .
$$

(ii) Para cada $\varepsilon>0$ y cada subconjunto finito $\left\{x_{1}^{*}, x_{2}^{*}, \ldots, x_{n}^{*}\right\}$ de $X^{*}$, existe una proyección $P$ en $X$ de rango finito $y$ norma uno $y$ un subconjunto finito $\left\{y_{1}^{*}, y_{2}^{*}, \ldots, y_{n}^{*}\right\}$ de $X^{*}$ verificando

$$
\left\|x_{i}^{*}-P^{*} y_{i}^{*}\right\| \leq \varepsilon, i=1,2, \ldots, n \text {. }
$$

Demostración. (i) $\Rightarrow$ (ii) Dado $\varepsilon>0$ y $\left\{x_{1}^{*}, x_{2}^{*}, \ldots, x_{n}^{*}\right\} \subseteq X^{*}$, llamamos $M$ al subespacio de $X^{*}$ generado por $\left\{x_{1}^{*}, x_{2}^{*}, \ldots, x_{n}^{*}\right\}$. Si $M=0$, lo que pretendemos demostrar es trivial; en otro caso, elegimos una base de $M$, que podemos suponer formada por $\left\{x_{1}^{*}, x_{2}^{*}, \ldots, x_{k}^{*}\right\}$, para algún natural $k \leq n$. Elegimos $x_{i} \in X \quad(i \leq k)$ tales que

$$
x_{i}^{*}\left(x_{i}\right)=1, \quad x_{i}^{*}\left(x_{j}\right)=0, \quad \text { si } i \neq j .
$$


Entonces, definimos el operador $T \in L(X)$ por

$$
T x=\sum_{i=1}^{k} x_{i}^{*}(x) x_{i} \quad(x \in X)
$$

$y$, por tanto,

$$
T^{*}=\sum_{i=1}^{k} x_{i} \otimes x_{i}^{*},
$$

y como $\left\{x_{1}, \ldots, x_{k}\right\}$ son linealmente independientes, entonces existe $z_{i}^{*} \in X^{*}$ tal que $T^{*}\left(z_{i}^{*}\right)=x_{i}^{*}$, para cada $i \leq n$. Fijamos

$$
K>\max \left\{\left\|z_{i}^{*}\right\|: 1 \leq i \leq n\right\}
$$

por (i) existe una proyección $P$ en $X$ de rango finito y $\|P\|=1$ de modo que

$$
\|T-T P\| \leq \varepsilon / K .
$$

Entonces, para cada $i \leq n$ se tiene

$$
\begin{gathered}
\left\|x_{i}^{*}-P^{*} T^{*} z_{i}^{*}\right\|=\left\|T^{*} z_{i}^{*}-P^{*} T^{*} z_{i}^{*}\right\| \leq \\
\leq\left\|T^{*}-P^{*} T^{*}\right\|\left\|z_{i}^{*}\right\| \leq \frac{\varepsilon}{K}\left\|z_{i}^{*}\right\| \leq \varepsilon
\end{gathered}
$$

y basta tomar

$$
y_{i}^{*}=T^{*} z_{i}^{*} \quad(1 \leq i \leq n)
$$

para que se verifique (ii).

(ii) $\Rightarrow$ (i) Sea $T \in L(X, F)$; existen subconjuntos $\left\{x_{1}, x_{2}, \ldots, x_{n}\right\}$ de $B_{X}$ y $\left\{x_{1}^{*}, x_{2}^{*}, \ldots, x_{n}^{*}\right\}$ de $X^{*}$ de forma que

$$
T x=\sum_{i=1}^{n} x_{i}^{*}(x) x_{i}, \quad \forall x \in X .
$$


Por hipótesis, hay una proyección $P$ con $\|P\|=1$ y rango finito, y un subronjunto $\left\{y_{1}^{*}, y_{2}^{*}, \ldots, y_{n}^{*}\right\}$ de $X^{*}$ verificanúo

$$
\left\|x_{i}^{*}-P^{*} y_{i}^{*}\right\| \leq \frac{\varepsilon}{2 n}, \quad \forall i \leq n
$$

Por tanto, si $x \in B_{X}$,

$$
\begin{gathered}
\|T x-T P x\|=\left\|\sum_{i=1}^{n}\left(x_{i}^{*}(x)-x_{i}^{*}(P x)\right) x_{i}\right\| \leq \\
\leq \sum_{i=1}^{n}\left|x_{i}^{*}(x)-x_{i}^{*}(P x)\right| \leq \\
\leq \sum_{i=1}^{n}\left[\left|x_{i}^{*}(x)-y_{i}^{*}(P x)\right|+\mid y_{i}^{*}(P(P x))-x_{i}^{*}(P x) \| \leq\right. \\
\leq 2 n \max \left\{\left\|x_{i}^{*}-P^{*} y_{i}^{*}\right\|: 1 \leq i \leq n\right\} \leq \varepsilon .
\end{gathered}
$$

5.36 Corolario. Sea $X$ un espacio de Banach verificando una cualquiera de las dos condiciones de la Proposición 5.35. Entonces, para cualquier espacio de Banach $Y$, el conjunto de los operadores de rango finito de $X$ en $Y$ que alcanzan su norma es denso en el espacio de los operadores de rango finito $F R(X, Y)$.

Demostración. Dados $T \in F R(X, Y)$ y $\varepsilon>0$, por hipótesis existe $P \in L(X)$, proyección de rango finito y norma uno tal que

$$
\|T-T P\| \leq \varepsilon .
$$

Por tanto, $T P \in F R(X, Y)$ y como $\|P\|=1$,

$$
P\left(b_{X}\right)=B_{P(X)}
$$


luego $T P$ alcanza su norma en $X$, ya que

$$
\|T P\|=\sup \{\|T x\|: x \in P(X),\|x\| \leq 1\}
$$

y $B_{P(X)}$ es compacta.

5.37 Proposición. Si $X=L_{1}(\mu)$ ( $\mu$ medida positiva y finita) o $C(K)$ (K espacio topológico compacto de Hausdorff), entonces

$$
\overline{F R N A(X, Y)} \supseteq F R(X, Y) \text {, }
$$

para todo espacio de Banach $Y$.

Demostración. Suponemos que $\mu$ es una medida sobre el conjunto $\Omega$. Probemos que satisface la condición (ii) de la Proposición 5.35. Sea $\varepsilon>0$ y $\left\{x_{1}^{*}, x_{2}^{*}, \ldots, x_{n}^{*}\right\} \subseteq\left(L_{1}(\mu)\right)^{*}$ tal que cada funcional $x_{i}^{*}$ viene representado por una función $g_{i} \in L_{\infty}(\mu)(i \leq n)$. Como el subespacio

$$
\operatorname{lin}\left\{\chi_{E}: E \text { medible }\right\} \text {, }
$$

es denso en $L_{\infty}(\mu)$, existe una familia de subconjuntos de $\Omega$ medibles y disjuntos $\left\{A_{1}, A_{2}, \ldots, A_{m}\right\}$ y escalares $\alpha_{j}^{i}, j \leq m, i \leq n$ de forma que si

$$
s_{i}:=\sum_{j=1}^{m} \alpha_{j}^{i} \chi_{A_{j}},
$$

entonces

$$
\left\|g_{i}-s_{i}\right\|_{\infty}<\varepsilon, \quad i \leq n
$$

Definimos entonces

$$
\begin{gathered}
P: L_{1}(\mu) \longrightarrow L_{1}(\mu) \text { por } \\
P(f)=\sum_{j=1}^{m}\left(\frac{1}{\mu\left(A_{j}\right)} \int_{A_{j}} f d \mu\right) \chi_{A_{j}}, \quad \forall f \in L_{1}(\mu) .
\end{gathered}
$$


Es inmediato que $P$ es una proyección en $L_{1}(\mu)$ (las funciones $\chi_{A}$, $(j \leq m)$ están en $L_{1}(\mu)$ por ser la medida $\mu$ finita) de rango finito. Además, es claro que si $f \in L_{1}(\mu)$,

$$
\begin{aligned}
\|P(f)\|_{1} & =\int_{\Omega}\left|\sum_{j=1}^{m}\left(\frac{1}{\mu\left(A_{j}\right)} \int_{A_{j}} f d \mu\right) \chi_{A_{j}}\right| d \mu \leq \\
& \leq \sum_{j=1}^{m} \int_{A_{j}}|f| d \mu \leq\|f\|_{1},
\end{aligned}
$$

esto es, $\|P\|=1$. Si $y_{i}^{*}$ es el funcional asociado a $s_{i}, 1 \leq i \leq n$, tenemos para $f \in L_{1}(\mu)$ y $1 \leq i \leq n$

$$
\begin{gathered}
P^{*} y_{i}^{*}(f)=\int_{\Omega} s_{i} \sum_{k=1}^{m}\left(\frac{1}{\mu\left(A_{k}\right)} \int_{A_{k}} f d \mu\right) \chi_{A_{k}} d \mu= \\
=\int_{\Omega} \sum_{j=1}^{m} \alpha_{j}^{i} \chi_{A_{j}}\left(\frac{1}{\mu\left(A_{j}\right)} \int_{A_{j}} f d \mu\right) d \mu= \\
=\int_{\Omega} \sum_{j=1}^{m} \alpha_{j}^{i} \int_{A_{j}} f d \mu=\int_{\Omega}\left(\sum_{j=1}^{m} \alpha_{j}^{i} \chi_{A_{j}}\right) f d \mu= \\
=\int_{\Omega} s_{i} f d \mu=y_{i}^{*}(f),
\end{gathered}
$$

i.e.,

$$
P^{*} y_{i}^{*}=y_{i}^{*}, 1 \leq i \leq n
$$

Así pues, la desigualdad

$$
\left\|g_{i}-s_{i}\right\|_{\infty} \leq \varepsilon, i \leq n,
$$

nos da la condición (ii) de la Proposición 5.35, puesto que

$$
\left\|x_{i}^{*}-P^{*} y_{i}^{*}\right\|=\left\|x_{i}^{*}-y_{i}^{*}\right\|=\left\|g_{i}-s_{i}\right\|_{\infty}<\varepsilon, \quad i=1,2, \ldots, n .
$$


El espacio $C(K)$ también satisface la condición (ii) de la Proposición 5.35: sean $\varepsilon>0$ y $\left\{\mu_{1}, \mu_{2}, \ldots, \mu_{n}\right\} \subseteq \mathcal{M}(K)$. Definimos

$$
\mu:=\sum_{i=1}^{n}\left|\mu_{i}\right|
$$

Dado $i \in\{1,2, \ldots, n\}$, y puesto que la medida $\mu_{i}$ es absolutamente continua con respecto a $\mu$, en virtud del Teorema de Radon-Nikodym existe $g_{i} \in L_{1}(\mu)$ tal que

$$
d \mu_{i}=g_{i} d \mu
$$

Como el subespacio

$$
\operatorname{lin}\left\{\chi_{E}: E \text { es medible }\right\}
$$

es denso en $L_{1}(\mu)$, hay una familia finita $\left\{A_{1}, A_{2}, \ldots, A_{m}\right\}$ de subconjuntos Borel-medibles y disjuntos, con $0<\mu\left(A_{j}\right), j \leq m$, y escalares $\alpha_{j}^{i}$, para $i \leq n$ de forma que la función

$$
s_{i}:=\sum_{j=1}^{m} \alpha_{j}^{i} \chi_{A}
$$

verifica

$$
\left\|g_{i}-s_{i}\right\|_{1}<\varepsilon / 2, \quad i \leq n .
$$

Por la regularidad de $\mu$, dado $j \in\{1,2, \ldots, m\}$, existe $\kappa_{j}$, subconjunto compacto de $K$, tal que

$$
K_{j} \subseteq A_{j}, \quad \mu\left(K_{j}\right)>0 \quad \text { y } \quad \mu\left(A_{j} \backslash K_{j}\right)<\frac{\varepsilon}{2 m M},
$$

donde $M>\max \left\{\left|\alpha_{j}^{i}\right|: i \leq n, j \leq m\right\}$. Considérese el subconjunto $\left\{\varphi_{1}, \varphi_{2}, \ldots, \varphi_{m}\right\} \subseteq C(K)$ de modo que $\varphi_{i \mid K_{j}}=0$, si $i \neq j$ y $\varphi_{i \mid K_{i}}=1$ y definamos

$$
\begin{gathered}
P: C(K) \longrightarrow C(K) \text { por } \\
P(f)=\sum_{j=1}^{m}\left(\frac{1}{\mu\left(K_{j}\right)} \int_{K_{j}} f d \mu\right) \varphi_{j}, \quad(f \in C(K)) .
\end{gathered}
$$


$P$ es una proyección de rango finito, norma uno y verifica

$$
P^{*}\left(\chi_{K_{h}} \mu\right)=\chi_{K_{h}} \mu \text {, }
$$

ya que si $f \in C(K)$, tenemos

$$
\begin{gathered}
P^{*}\left(\chi_{K_{h}} \mu\right)(f)=\int_{K_{h}} P(f) d \mu= \\
=\sum_{j=1}^{m}\left(\frac{1}{\mu\left(K_{j}\right)} \int_{K_{j}} f d \mu\right) \int_{K_{h}} \varphi_{j} d \mu=\int_{K_{h}} f d \mu=\chi_{K_{h}} \mu(f) .
\end{gathered}
$$

Por tanto, definiendo $u_{i}:=\left(\sum_{j=1}^{m} \alpha_{j}^{i} \chi_{K}\right) \mu$, entonces $P^{*} u_{i}=u_{i}$ y usando que $d \mu_{i}=g_{i} d \mu(1 \leq i \leq n)$, obtenemos

$$
\begin{gathered}
\left\|\mu_{i}-P^{*} u_{i}\right\| \leq\left\|\mu_{i}-s_{i} \mu\right\|+\left\|s_{i} \mu-u_{i}\right\| \leq \\
\leq \int_{K}\left|g_{i}-s_{i}\right| d \mu+\int_{K}\left|s_{i}-\sum_{j=1}^{m} \alpha_{j}^{i} \chi_{K}\right| d \mu \leq \\
\leq \varepsilon / 2+\sum_{j=1}^{m}\left|\alpha_{j}^{i}\right| \mu\left(A_{j} \backslash K_{j}\right) \leq \varepsilon .
\end{gathered}
$$

Demostración del Teorema 5.28. Si $X=C(K)$, entonces $X^{*} \equiv \mathcal{M}(K)$ es un espacio del tipo $L_{1}(\mu)$ (véase [36, Theorem 8.5]). En caso de que $X=L_{1}(\mu)$, para $\mu$ finita, entonces $X^{*}$ se identifica, usando el Teorema de Gelfand-Naimark, con un espacio tipo $C(S)$. La Proposición 5.32 nos asegura entonces que si $X=C(K) \circ X=L_{1}(\mu), X^{*}$ tiene la P.A., y, en virtud de la Proposición 5.34, el conjunto $F R(X, Y)$ es denso en $K(X, Y)$, para cualquier espacio de Banach $Y$. Basta usar la Proposición 5.37 para concluir que $F R N A(X, Y)$ es denso en el conjunto de todos los operadores de rango finito de $X$ en $Y$.

El Teorema 5.29 tiene una demostración más directa, basada en el siguiente resultado: 
5.38 Proposición ([24, Lemma 3.4]). Sea $Y$ un espacio de Banach verificando que para todo $\varepsilon>0$ y cada subconjunto $\left\{y_{1}, y_{2}, \ldots, y_{n}\right\}$ de $Y$ existe $E$ subespacio de $Y$ de dimensión finita con la propiedad $B$ y tal que, para cada $i \in\{1,2, \ldots, n\}$, existe $e_{i} \in E$ de modo que

$$
\left\|y_{i}-e_{i}\right\|<\varepsilon
$$

y una proyección $P$ de $Y$ sobre $E$ de norma uno. Entonces, para todo espacio de Banach $X$, el conjunto $F R N A(X, Y)$ es denso en $K(X, Y)$.

Demostración. Fijamos un operador compacto $T \in K(X, Y)$ y un número real positivo $\eta$. Por ser $T$ compacto, existe un subconjunto $\left\{y_{1}, y_{2}, \ldots, y_{n}\right\}$ de $Y$ de modo que para cada $x \in B_{X}$,

$$
\min \left\{\left\|T x-y_{i}\right\|: i \leq n\right\}<\eta / 8 .
$$

Por hipótesis, existe un subespacio $E$ de $Y$ con la propiedad $B$, un subconjunto $\left\{e_{1}, e_{2}, \ldots, e_{n}\right\}$ de $E$ y una proyección $P$ de $Y$ en $E$ verificando las condiciones del enunciado para $\varepsilon=\eta / 8$. Si $x \in B_{X}$, entonces existe $i \leq n$ tal que

$$
\left\|T x-y_{i}\right\| \leq \eta / 8
$$

luego

$$
\left\|T x-e_{i}\right\| \leq\left\|T x-y_{i}\right\|+\left\|y_{i}-e_{i}\right\| \leq \eta / 4
$$

de donde

$$
\|T x-P T x\| \leq\left\|T x-e_{i}\right\|+\left\|P e_{i}-P T x\right\| \leq \eta / 4+\eta / 4=\eta / 2
$$

y por tanto,

$$
\|T-P T\| \leq \eta / 2 .
$$

Pero $P T \in L(X, E)$ y $E$ tiene la propiedad $B$, así que existe un operador $T_{0} \in F R N A(X, Y)$ tal que

$$
\left\|P T-T_{0}\right\| \leq \eta / 2
$$


y de ahí

$$
\left\|T-T_{0}\right\| \leq \eta
$$

Demostración del Teorema 5.29. Probemos que si $Y=L_{1}(\mu)$, con $\mu$ una medida positiva arbitraria, verifica las hipótesis de la proposición anterior. Sea $\varepsilon>0$ y $\left\{f_{1}, f_{2}, \ldots, f_{n}\right\} \subseteq L_{1}(\mu)$. Dado $i=1,2, \ldots, n$, existe $s_{i} \in \operatorname{lin}\left\{\chi_{A}: A\right.$ medible y $\left.0<\mu(A)<\infty\right\}$ tal que

$$
\left\|f_{i}-s_{i}\right\|<\varepsilon \text {. }
$$

Sea $\left\{A_{1}, A_{2}, \ldots, A_{m}\right\}$ una familia finita de conjuntos medibles y disjuntos verificando $0<\mu\left(A_{j}\right)<\infty, j=1,2, \ldots, m$ y de forma que para ciertos escalares $\alpha_{j}^{i}$,

$$
s_{i}=\sum_{j=1}^{m} \alpha_{j}^{i} \chi_{A_{j}}
$$

Definimos

$$
\begin{gathered}
P: L_{1}(\mu) \longrightarrow \operatorname{lin}\left\{\chi_{A_{i}}: i \leq m\right\} \\
f \mapsto P(f)=\sum_{j=1}^{m}\left(\frac{1}{\mu\left(A_{j}\right)} \int_{A_{j}} f d \mu\right) \chi_{A_{j}} .
\end{gathered}
$$

Es claro que $P$ es una proyección de $L_{1}(\mu)$ sobre $E:=\operatorname{lin}\left\{\chi_{A_{i}}: i \leq m\right\}$ de norma uno. Además, en virtud de (1), para cada $i \in\{1,2, \ldots, n\}$ existe $s_{i} \in E$ tal que $\left\|f_{i}-s_{i}\right\|<\varepsilon$ y $E$ tiene la propiedad $B$, puesto que la aplicación lineal

$$
\Phi: E \longrightarrow l_{1}^{m}
$$

que verifica

$$
\Phi\left(\frac{\chi_{A_{j}}}{\mu\left(A_{j}\right)}\right)=e_{j}, \quad(j=1,2, \ldots, m)
$$

( $e_{j}$ es el $j$-ésimo vector de la base usual de $\mathbb{R}^{m}$ ) es un isomorfismo isométrico y el espacio $l_{1}^{m}$ tiene la propiedad $B$ (recuérdese que trabajamos con el espacio de funciones reales $\left.L_{1}(\mu)\right)$. 
También el espacio $C(K)$ ( $K$ espacio topológico compacto y de Hausdorff) verifica las hipótesis de la Proposición 5.38. Sea $\varepsilon>0$ y $\left\{f_{1}, f_{2}, \ldots, f_{n}\right\} \subseteq C(K)$ y $\varepsilon>0$. Consideremos un recubrimiento de $K$ por abiertos $\left\{U_{1}, U_{2}, \ldots, U_{m}\right\}$ de forma que si $j \in\{1,2, \ldots, m\}$, entonces

$$
t, s \in U_{j} \Rightarrow\left|f_{i}(t)-f_{i}(s)\right|<\varepsilon / m,
$$

cualquiera que sea $i \in\{1,2, \ldots, n\}$. Tomamos $\left\{\varphi_{1}, \varphi_{2}, \ldots, \varphi_{m}\right\}$ una partición de la unidad asociada al recubrimiento $\left\{U_{1}, U_{2}, \ldots, U_{m}\right\}$ y para cada $j \leq m$ elegimos un elemento $t_{j} \in U_{j}$. Basta tomar

$$
E:=\operatorname{lin}\left\{\varphi_{1}, \varphi_{2}, \ldots, \varphi_{m}\right\}
$$

y la aplicación

$$
P: C(K) \longrightarrow E
$$

definida por

$$
P(f)=\sum_{j=1}^{m} f\left(t_{j}\right) \varphi_{j}, \quad(f \in C(K))
$$

Es inmediato que $P$ es una proyección de $C(K)$ sobre $E$, de norma uno. Si $i \in\{1,2, \ldots, n\}$, el elemento $s_{i}:=P\left(f_{i}\right)$ verifica

$$
\left\|f_{i}-s_{i}\right\|<\varepsilon
$$

ya que dado $t \in K$, si notamos $F_{:}=\left\{k \leq m: t \in U_{k}\right\}$, entonces

$$
\begin{gathered}
\left|f_{i}(t)-\sum_{j=1}^{m} f_{i}\left(t_{j}\right) \varphi_{j}(t)\right|=\left|\sum_{j=1}^{m}\left(f_{i}(t)-f_{i}\left(t_{j}\right)\right) \varphi_{j}(t)\right| \leq \\
\leq \sum_{j \in F_{t}}\left|f_{i}(t)-f_{i}\left(t_{j}\right)\right| \varphi_{j}(t) \leq \varepsilon \sum_{j=1}^{m} \varphi_{j}(t)=\varepsilon .
\end{gathered}
$$

Como la aplicación

$$
\Phi: E \longrightarrow l_{\infty}^{m}
$$




$$
\Phi\left(\varphi_{j}\right)=e_{j},(j=1,2, \ldots, m)
$$

extendida por linealidad, es un isomorfismo isométrico, y $l_{\infty}$ tiene la propiedad $B$, entonces $E$ tiene la propiedad $B$.

En esta sección hemos probado que para ciertos espacios espacios clásicos se verifica la densidad de los operadores compactos (de hecho de rango finito) que alcanzan la norma en el conjunto de todos los operadores compactos. Parece entonces natural preguntarse qué ocurre en general:

5.39 Problema ([24, Question 2]). ¿Existen dos tspacios de Banach $X$ e $Y$ para los cuales el conjunto $K N A(X, Y)$ no sea denso en $K(X, Y)$ ?

$\mathrm{Ni}$ aún en el caso en que $Y$ sea finito dimensional, la cuestión anterior tiene respuesta, es decir, no conocemos ejemplo alguno de espacio de dimensión finita que no tenga la propiedad $B$. 


\section{Bibliografía}

[1] M.D. Acosta, Operadores que alcanzan su radio numérico, Tesis Doctoral, Univ. Granada, 1990.

[2] M.D. Acosta, F.J. Aguirre and R. Payá, A space by W. Gowers and new results on norm and numerical radius attaining operators, Acta Univ. Carolinae Math. et Phys. 33 (1992), 5-14.

[3] M.D. Acosta, F.J. Aguirre and R. Payá, A new sufficient condition for the denseness of norm attaining operators, Preprint, Univ. Granada (1993).

[4] E. Bishop and R.R. Phelps, A proof that every Banach space is subreflexive, Bull. Amer. Math. Soc. 67 (1961), 97-98.

[5] E. Bishop and R.R. Phelps, The support functionals of a convex set, Proc. Sympos. Pure Math., Vol VII: Convexity, 27-35. Amer. Math Soc., Providence, R.I., (1963).

[6] B. Bollobás, An extension of the theorem of Bishop and Phelps, Bull. London Math. Soc. 2 (1970), 181-182.

[7] J. Bourgain, Strongly exposed points in weakly compact convex sets in Banach spaces, Proc. Amer. Math. Soc. 58 (1976), 197-200. 
[8] J. Bourgain, On dentability and the Bishop-Phelps property, Israel J. Math. 28 (1977), 265-271.

[9] R. Deville, G. Godefroy and V. Zizler, Smoothness and renormings in Banach spaces, Longman Scientific and Technical, New York, 1.993.

[10] J. Diestel, Sequences and series in Banach spaces, Springer-Verlag, New York, 1984.

[11] J. Diestel and J. Uhl, Vector Measures, Amer. Math. Soc., Math. Surveys No.15, Providence, R. I., 1977.

[12] J. Dugundji, Topology, Allyn and Bacon, Inc., Boston, 1.968.

[13] N. Dunford and J. Schwartz, Linear Operators. Part I: General Theory, Interscience, New York, 1957.

[14] J.R. Giles, Convex analysis with application in differentiation of convex functions, Pitman Advanced Publishing Program, Boston, 1982.

[15] B.V. Godun y S.L. Troyanski, Operators attaining their norms and the geometry of the unit sphere of a Banach space, Soviet Math. Dokl. 42 (1991), 532-534.

[16] W. Gowers, Symmetric block bases of sequences with large average growth, Israel J. Math. 69 (1990), 129-149.

[17] S. Guerre-Delabrière, Classical sequences in Banach spaces, Marcel Dekker, Inc., New York, 1992.

[18] M. Guzman y B. Rubio, Integración: teoria y técnicas, Alhambra, Madrid, 1979.

[19] R. Huff, On non-density of norm attaining operators, Rev. Ruum. Math. Pures et Appl. 25 (1980), 239-241. 
[20] R.E. Huff and P.D. Morris, Geometric characterizations of th: RNP in Banach spaces, Studia Math. 66 (1976), 157-164.

[21] A. Iwanik, Norm attaining operators on Lebesgue spaces, Pac. J. Math. 83 (1979), 381-386.

[22] R.C. James, Reflexivity and the supremum of linear functionals, Ann. of Math. 66 (1957), 159-169.

[23] G. Jameson, Topology and normed spaces, Chapman and Hall, London, 1974.

[24] J. Johnson y J. Wolfe, Norm attaining operators, Studia Math. 65 (1979), 7-19.

[25] J. Johnson y J. Wolfe, Norm attaining operators and simultaneously continuos retractions, Proc. Amer. Soc. 86 (1982), 609-612.

[26] V. Klee, Extremal structure of convex sets II, Math. Z. 69 (1958), 90-104.

[27] J. Lindenstrauss, On operators which attain their norm, Israel J. Math. 1 (1963), 139-148.

[28] J. Lindenstrauss and L. Tzafriri, Classical Banach Spaces I, Springer-Verlag, Berlin, 1977.

[29] J.R. Partington, Norm attaining operators, Israel J. Math. 43 (1982), 273-276.

[30] R.R. Phelps, Subreflexive normed spaces, Arch. Math. 8 (1957), 444450.

[31] R.R. Phelps, The Bishop-Phelps Theorem in complex spaces: an open problem, Function spaces, Lecture Notes in Mathematics 36, K. Jarosz (ed.), Dekker, New York (1.992), 337-340. 
[32] R.R. Phelps, Convex Functions, Monotone Operators and Differentiability, Springer-Verlag, Berlin, 1993.

[33] R.A. Poliquin and V.E. Zizler, Optimization of convex functions on $w^{*}$-compact sets, Manuscripta Math. 68 (1990), 249-270.

[34] W. Schachermayer, Norm attaining operators and renormings of Banach spaces, Israel J. Math. 44 (1983), 201-212.

[35] W. Schachermayer, Norm attaining operators on some classical Banach spaces, Pac. J. Math. 105 (1983), 427-438.

[36] H.H. Schaefer, Banach lattices and positive operators, SpringerVerlag, New York, 1974.

[37] S. Shelah, Notices Amer. Soc. 26 (1979), A-525.

[38] C. Stegall, Optimization and differentiation in Banach spaces, Linear Alg. and Appl. 84 (1986), 191-211.

[39] J. Uhl, Norm attaining operators in $L_{1}[0,1]$ and the R.N.P., Pacific J. Math. 63 (1976), 293-300.

[40] V. Zizler, On some extremal problems in Banach spaces, Math. Scand. 32 (1973), 214-224. 